\title{
KEGG orthology-based annotation of the predicted proteome of Acropora digitifera: ZoophyteBase - an open access and searchable database of a coral genome
}

Walter C Dunlap ${ }^{1,2}$, Antonio Starcevic ${ }^{4}$, Damir Baranasic ${ }^{4}$, Janko Diminic ${ }^{4}$, Jurica Zucko ${ }^{4}$, Ranko Gacesa ${ }^{4}$, Madeleine JH van Oppen ${ }^{1}$, Daslav Hranueli ${ }^{4}$, John Cullum ${ }^{5}$ and Paul F Long ${ }^{2,3^{*}}$

\begin{abstract}
Background: Contemporary coral reef research has firmly established that a genomic approach is urgently needed to better understand the effects of anthropogenic environmental stress and global climate change on coral holobiont interactions. Here we present KEGG orthology-based annotation of the complete genome sequence of the scleractinian coral Acropora digitifera and provide the first comprehensive view of the genome of a reef-building coral by applying advanced bioinformatics.

Description: Sequences from the KEGG database of protein function were used to construct hidden Markov models. These models were used to search the predicted proteome of A. digitifera to establish complete genomic annotation. The annotated dataset is published in ZoophyteBase, an open access format with different options for searching the data. A particularly useful feature is the ability to use a Google-like search engine that links query words to protein attributes. We present features of the annotation that underpin the molecular structure of key processes of coral physiology that include (1) regulatory proteins of symbiosis, (2) planula and early developmental proteins, (3) neural messengers, receptors and sensory proteins, (4) calcification and $\mathrm{Ca}^{2+}$-signalling proteins, (5) plant-derived proteins, (6) proteins of nitrogen metabolism, (7) DNA repair proteins, (8) stress response proteins, (9) antioxidant and redox-protective proteins, (10) proteins of cellular apoptosis, (11) microbial symbioses and pathogenicity proteins, (12) proteins of viral pathogenicity, (13) toxins and venom, (14) proteins of the chemical defensome and (15) coral epigenetics.

Conclusions: We advocate that providing annotation in an open-access searchable database available to the public domain will give an unprecedented foundation to interrogate the fundamental molecular structure and interactions of coral symbiosis and allow critical questions to be addressed at the genomic level based on combined aspects of evolutionary, developmental, metabolic, and environmental perspectives.
\end{abstract}

Keywords: Acropora digitifera, KEGG orthology, Database, Annotation, Proteome, Genome, Coral, Symbiosis, Cnidaria

\footnotetext{
*Correspondence: paul.long@kcl.ac.uk

${ }^{2}$ Institute of Pharmaceutical Science, King's College London, Franklin-Wilkins

Building, 150 Stamford Street, London SE1 9NH, United Kingdom

${ }^{3}$ Department of Chemistry King's College London, Franklin-Wilkins Building,

150 Stamford Street, London SE1 9NH, United Kingdom

Full list of author information is available at the end of the article
} 


\section{Background}

All of the reef-building corals (Scleractinia; phylum Cnidaria) that create the vast calcium carbonate deposits of coral reefs have evolved an endosymbiotic partnership with photosynthetic dinoflagellates of the genus Symbiodinium (Dinophyceae), commonly known as zooxanthellae, which reside within the gastrodermal cells of their scleractinian host [1-3]. Coral-algal symbiosis is a cooperative metabolic adaptation necessary for survival in the shallow oligotrophic (nutrient-poor) waters of tropical and subtropical marine environments $[4,5]$ that drives the productivity of coral reefs [6]. Coral reefs provide habitat and trophic support for many thousands of marine species, the richness of which rival the biological biodiversity of tropical rainforests [7]. Underlying the basic requirements of corals for growth, reproduction and survival are special needs to accommodate symbiont-specific host recognition, to control innate and responsive immune systems, and what is likely to emerge from future research is the extent to which the host is involved in direct regulation of its endosymbiont populations. Much is understood about the cellular biology of cnidarian-dinoflagellate symbiosis (reviewed in [8]), but less is known at the molecular level of coral symbiology. There is little opposition to the contention that environmental and anthropogenic disturbances are causing alarming losses to coral reefs ([9] and reference therein). Threats to productivity are being imposed by the disruption of coral symbiosis (apparent as "coral bleaching") caused in response to increasing thermal stress attributed to global warming $[10,11]$, from an increase in stress-related coral disease [12-14], from the discharge of domestic and industrial wastes, pollutants from agricultural development and the transport of sediments in terrestrial runoff $[15,16]$, and potentially from imminent declines in coral calcification owing to rising ocean acidification [17-19]. Accordingly, we require a better understanding of the molecular stress responses and adaptive potential of corals. Such information is necessary to predict bleaching events and so better inform effective management policies for the conservation of coral reef ecosystems [20-24].

To understand how coral holobionts respond to environmental change at the molecular level, the identification of genes that may respond by transcription to stress is of primary importance [25]. Thus, the use of transcriptomic methodologies to identify stress-responsive genes has been highly successful [26-32]. Transcriptome high-throughput profiling has allowed changes in gene expression across thousands of genes to be measured simultaneously. Fuelled by data-generating power, the number of coral based studies utilising transcriptomics to investigate molecular responses to environmental stressors has expanded greatly by the acquisition of expressed sequence tag (EST) gene libraries, the fabrication of microarray biochips used to estimate levels of mRNA expression, and by direct analysis using next-generation, high-throughput sequencing. However, much of this work has been conducted using the aposymbiotic state of pre-settlement coral larvae, so transcribed genes relevant to metamorphosis and the cytobiology of the adult polyp are limited to a few recent studies [33-36]. The transcriptome additionally does not provide the structural framework and essential regulatory elements of the functional genome for comprehensive evaluation. Recently, deep metatranscriptomic sequencing of two adult coral holobiomes has been made available on searchable databases: PocilloporaBase for Pocillopora damicornis [36] and PcarnBase for Platygyra carnosus [37]. In contrast, high-throughput metaproteomic analyses to quantify the product yield of stress-response genes of the coral holobiome are yet to be widely adopted by the coral reef scientific community, despite the proteome being the ultimate measure of the coral phenotype [38,39].

The early accumulation of transcriptomic data revealed that a small proportion of coral ESTs matched genes known previously only from other kingdoms of life, implying that the ancestral animal genome contained many genes traditionally regarded as 'non-animal' that have been lost from most animal genomes [40]. Furthermore, an unexpected revelation from EST data is the greater extent to which coral sequences resemble human genes than those of the Drosophila and Caenorhabditis model invertebrate genomes [41,42]. Comparative genomic analysis has revealed higher genetic divergence and massive gene loss within the ecdysozoan lineages. Hence, many genes assumed to have much later evolutionary origins are likely to have been present in an ancestral or early-diverged metazoan [43]. While much of the animal kingdom remains yet to be explored, examples of the metazoan phylum Cnidaria provide a unique insight into the deep evolutionary origins of at least some vertebrate gene families [42]. Thus, the complete genomic sequence of a coral is likely to reveal many genes previously assumed to be strictly vertebrate innovations. To date, cnidarian genomes have been published for the sea anemone $N$. vectensis [42] and the hydroid Hydra magnipapillata [44]. Only the coral genome of Acropora digitifera is available without restriction on use of its published sequence [45], but the compiled sequence has not been fully annotated. At the time of this writing, the genome assembly of Acropora millepora has been released to the public domain [46], also without full annotation, but an embargo is imposed on use of this data that is highly restrictive to the progress of further studies. Understanding how genomic variation affects molecular and organismal biology is the ultimate justification of genome sequencing, and annotation is an essential step in this process. We envisage that unrestricted access to annotation of the A. digitifera genome will provide an unprecedented foundation to freely 
interrogate the generic molecular structure, possible endobiotic interactions and the response of coral to environmental stress. Accordingly, we offer annotation of the predicted proteome of $A$. digitifera on the open access and searchable database, ZoophyteBase [47]. Use of the ZoophyteBase search engines will allow genes of encoded proteins to be identified that can be examined in context of the cellular physiology, processes of ecological significance, the evolutionary and developmental biology of corals and the functional metabolism of the holobiont that collectively underpin the health of coral reefs.

\section{Construction and content}

ZoophyteBase is an open access and searchable database of complete annotation of the predicted proteome of the coral A. digitifera [48]. It was constructed using the MEGGASENSE system, which is a general system for constructing annotation databases with different sorts of input data (DNA reads, assembled genomes, predicted proteomes) and the possibility of using different combinations of analysis tools to create the annotation (Gacesa et al, in preparation). In the case of ZoophyteBase, hidden Markov model (HMM) profiles [49] were chosen as the annotation tool rather than the more common BLAST searches [50]. HMM profiles are constructed from multiple alignments of protein families and contain information about conserved differences in amino acid residues as well as deletions and insertions [49]. This is particularly important for a coral database, as corals are evolutionarily distant to most other organisms. This means that known homologous sequences present in the databases will usually have relatively low similarity, making BLAST searches inaccurate. The statistical information in an HMM profile gives more sensitive and accurate detection of sequence homology. An additional advantage of HMM profiles is that the statistical significance of hits (the expected value) is much more accurate than that calculated by BLAST programs.

The quality of sequence annotation is limited by the accuracy of information provided in any database used. It is well known that there are many problems with annotation in the large uncurated databases such as the NCBI GenBank nr sequences. Widely accepted, the most accurate database for functional annotation is the KEGG database [51]. The KEGG database organises sequences as groups of KEGG orthologues. These are sets of homologous sequences from as wide a range of organisms as possible having an assigned molecular function. These functions are arranged in a hierarchical fashion and grouped in biological pathways. The sequences belonging to KEGG orthologues were used to construct HMM profiles for annotating the coral sequences. Accordingly, the 23,524 predicted proteins encoded in the coral genome were analysed using HMM profiles. If a protein showed a highly significant correlation ("hit") to a single HMM profile, this was used to create a "trusted" annotation of the sequence. Choosing a cut-off for this criterion is not trivial, because longer sequences tend to have more significant e-values. For construction of ZoophyteBase the criterion 1e-5 was used. This resulted in 19,044 predicted proteins giving "trusted" sequence annotation. For many of these proteins there were two or more highly significant hits to established HMM profiles. In these cases, the most significant correlation was used to construct our "best-fit" annotation file, but other hits can be viewed by the database user so that expert knowledge can be employed to override the automatic annotation function. In 8,004 out of 19,044 predicted proteins which were annotated, more than one annotation was assigned based on nonoverlapping regions within the protein which were used to construct the "best-fit" annotation file. We interpreted these as "fusion" events generated by the in silico protein prediction method used, and these proteins were treated as multiple instead of single encoded proteins. Hence, this analysis resulted in the annotation of 33,195 proteins in total, generated from the original 23,524 predicted coral proteins. This is a very conservative annotation scheme, so it can be assumed that most of the annotations are biologically meaningful. Almost $81 \%$ (19,044 out of 23,524) of the predicted proteome was assigned using this method.

\section{Utility}

The MEGGASENSE system was used to generate a web interface for ZoophyteBase. The home page (Figure 1A) allows the use of several functions. A text version of the entire annotation can be downloaded for manual inspection. There is a proteome overview that gives statistics about the database and a breakdown of the annotated functions into different categories of genes. A particularly useful feature of ZoophyteBase is the ability to use text queries employing a search engine that provides a relevant inquiry in the absence of an exact match between key words of a search and those described for a functional protein. The search engine uses text from the KEGG-database, PubMed and other sources to establish links between query words to access protein data using an intelligent Google-like search engine implemented by the search platform Lucene/Solr [52]. This helps to overcome the common problem that different terminology is used by different groups of researchers. The use of this search function is illustrated by using the query "phagocytosis" (Figure 1B). This inquiry finds 42 hits to KEGG orthologue profiles. One of the hits corresponds to amphiphysin (a synaptic vesicle protein) with annotation of two protein homologues encoded in the coral genome. On the data page there is a brief description of the function of amphiphysin together with a PUBMED literature reference. The sequences of the predicted coral 


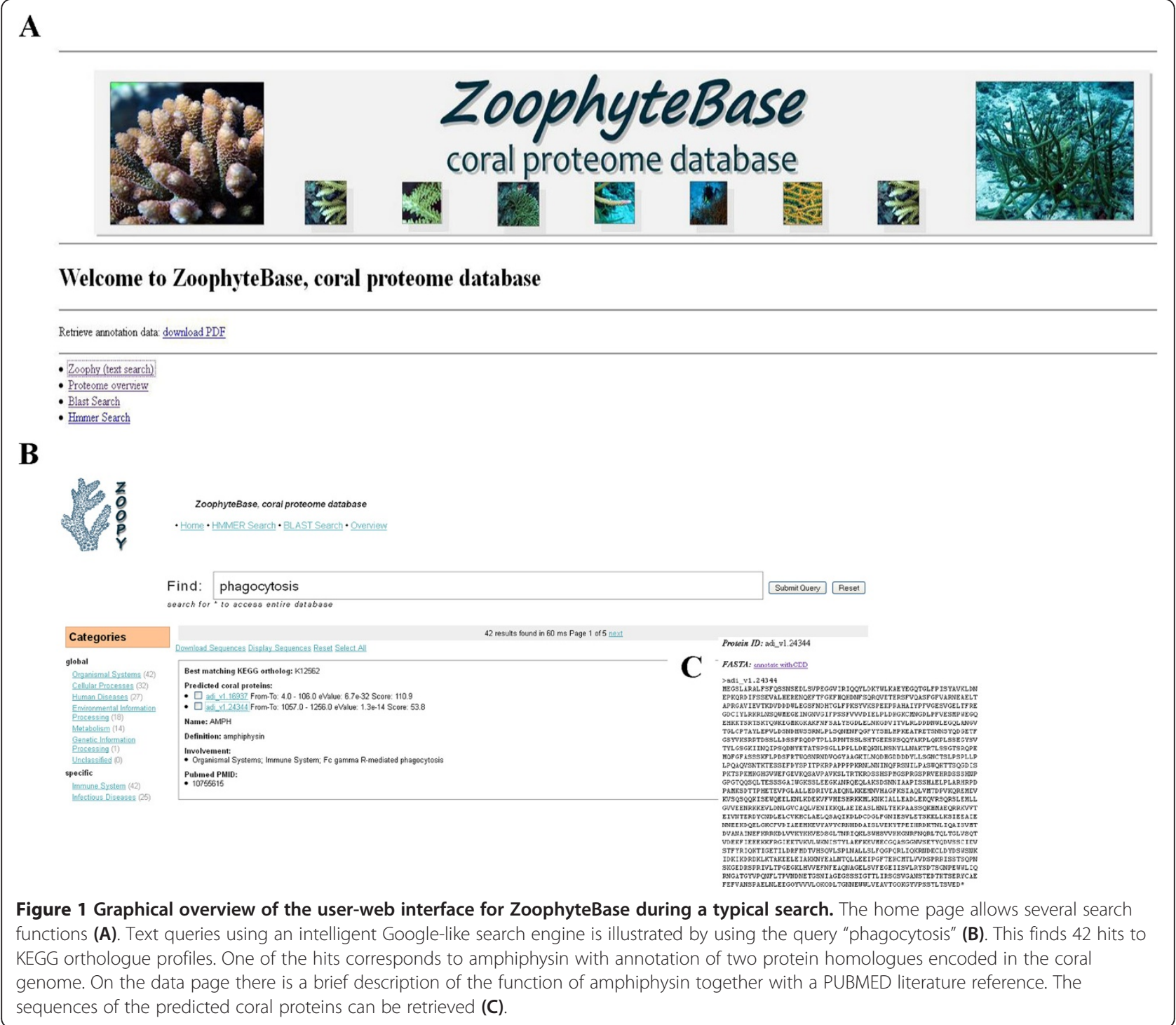

proteins (Figure 1C) can be retrieved, and it is also possible to analyse such data with computer aided drug design methods [53] to extract conserved domains. There are also two tools for the user to examine matches to protein sequences. The user can carry out a BLAST search against the coral protein sequence or analyse the predicted sequence against HMM profiles used to annotate the coral proteome. These tools require only the user to paste their queury into the sequence window.

In this manuscript we demonstrate the utility of ZoophyteBase by presenting predicted gene-encoded proteins revealed by annotation of the $A$. digitifera genome that have physiological, biological and environmental significance. We discuss features of importance in coral physiology: (1) regulatory proteins of symbiosis, (2) planula and early developmental proteins, (3) neural messengers, receptors and sensory proteins, (4) calcification and $\mathrm{Ca}^{2+}$ - signalling proteins, (5) plant-derived proteins, (6) proteins of nitrogen metabolism, (7) DNA repair proteins, (8) stress response proteins, (9) antioxidant and redox-protective proteins, (10) proteins of cellular apoptosis, (11) microbial symbioses and pathogenicity proteins, (12) proteins of viral pathogenicity, (13) toxins and venom, (14) proteins of the chemical defencesome and (15) coral epigenetics.

\section{Discussion}

\section{Regulatory proteins of symbiosis}

Metabolic cooperation is a key feature of coral-algal symbiosis that allows reef-building corals to inhabit the often nutrient-poor waters of tropical oceans [54]. In this phototropic symbiosis, fixed carbon produced by resident algae is released to the host for nutrition, and the algal symbionts benefit by acquiring the inorganic nutrient wastes of host metabolism $[2,55]$. The symbiotic 
dinoflagellates reside and proliferate within a specialised phagosome (the symbiosome) maintained within host gastrodermal cells. This arrangement requires complex biochemical coordination by the coral at various metabolic stages that includes endocytosis (phagocytosis) by postsettlement polyps to acquire algal symbionts, accord symbiosome recognition to arrest phagosomal maturation for sustained organelle homeostasis, activate symbiophagy or exocytosis to eliminate damaged symbionts $[56,57]$, and regulate apoptotic or exocytotic pathways to remove excess or impaired populations, all of which have long been recognised as essential to preserve the stability of coral symbiosis [58]. Although these processes are poorly understood in corals, it has been realised from studies of the sea anemone Aiptasia pulchella, a related anthozoan also containing Symbiodinium sp. endosymbionts, that the persistence of algal-containing symbiosomes in Cnidaria relies on the exclusion or retention of small Rab GTPase family proteins that are key regulatory components of vesicular trafficking and membrane fusion in eukaryotic cells [59]. Significantly, ApRab3 and ApRab4 accumulate in the biogenesis of maturing symbiosomes of $A$. pulchella $[60,61]$, and mature symbiosomes enveloping healthy dinoflagellates have tethered ApRab5 [62], a checkpoint antagonist of downstream ApRab7 and ApRab11 proteins that would otherwise direct autophagy of the symbiont cargo $[63,64]$.

Our annotation of the A. digitifera genome reveals sequences encoding putative Rab homologues of the Ras superfamily of proteins (Table 1). In a comparison of cnidarian Rab proteins, eight proteins of $A$. digitifera matched homologues of Aiptasia pulchella, twenty-nine matched proteins encoded by the aposymbiotic freshwater $H$. magnipapillata and the aposymbiotic anemone $N$. vectensis genomes, while seven Rab and Rab-interacting proteins of $A$. digitifera did not match other cnidarian proteins (Table 2). Significantly, the eight homologues of $A$. digitifera that matched exclusively Rab proteins of $A$. pulchella included homologues of the aforementioned ApRab3, ApRab4 and ApRab5 proteins attributed to the maintenance of healthy symbiosomes in Aiptasia, while homologues of the autophagic ApRab7 and ApRab11 proteins are found also in $N$. vectensis. While Rab GTPase and their effector proteins coordinate consecutive stages of endocytic vesicular transport $[65,66]$, soluble N-ethylmaleimide-sensitive factor attachment receptor (SNARE) proteins are essential for Rab assembly to complete endosomal fusion of vesicle membranes [67], a process by which Rab proteins impart specificity by binding distinct Rab and SNARE partner proteins prior to membrane fusion [68]. Genes encoding syntaxin-like SNARE proteins have been unambiguously identified [69] from coral EST database libraries constructed from expressed mRNA isolated from various early life stages of
Acropora aspera, A. millepora, A. palmata and Orbicella faveolata (= Monastraea faveolata), as well as from the genome of the sea anemone $N$. vectensis [70]. In metazoans, vacuolar r-SNARE receptor proteins comprise the syntaxin, synaptobrevin and VAMP family proteins, of which there are eight syntaxin and syntaxin-binding proteins (plus two plant-like syntaxins). Additionally, there are one t-SNARE target protein to direct vacuolar morphogenesis, two synaptosomal proteins, one synaptosomal complex ZIP1 protein (yeast homologue), one synaptobrevin membrane protein of secretory vesicles, ten vesicle-associated membrane proteins (VAMPs), a vacuolar protein-8 regulator of autophagy, four vacuolarsorting proteins and two SEC22 vesicle trafficking protein encoded in the genome of $A$. digitifera (Table 1 ), many of which may interact to provide metabolic transport between the endoplasmic reticulum and Golgi apparatus [71]. Included in this vast but yet unexplored repertoire of vacuolar-acting proteins are the syntaxinbinding amisyn and tomosyn regulators of SNARE complex assembly and disassembly [72,73], which may control membrane fusion in the phagocytic establishment and dissociation of coral symbiosis.

In the final step of exocytosis there is a cytosolic influx of calcium which binds to synaptotagmin to actuate completion of membrane SNARE protein assembly with exocytic docking to form the conducting channel for trans-membrane vesicular transport on activation by vesicle-fusing ATPase [74]. As synaptotagmin proteins are not included in the KEGG database, Zoophytebase was used for BLAST searches with all known synaptotagamin sequences [27]. Synaptotagamin proteins from $A$. digitifera were found having similarity to homologues from diverse invertebrate and vertebrate organisms, including one from the human genome (Table 3). Other $\mathrm{Ca}^{2+}$-sensing proteins of A. digitifera, such as calmodulin and the calcium binding protein $\mathrm{CML}$, are given with calcification and $\mathrm{Ca}^{2+}$-signalling proteins.

Intriguingly, annotation of the A. digitifera genome reveals a host cell factor (K14966), but this is not related to the elusive "host factor" of symbiosis demonstrated to be present in tissue homogenates of corals and other marine invertebrates that harbor Symbiodinium spp. endosymbionts [75-77]. Instead, this mammalian transcriptional coactivator host cell factor (HFC-1) is known to mediate the enhancer-promoter assemblies of herpes simplex (HSV) and varicella zoster (VZV) viruses for activation of the latent state for replication [78], such that the coral HCF homologue may have similar relevance as a viral checkpoint transcriptional coactivator of virulence in A. digitifera. HCF-1 expression is coupled also to chromatin modification $[79,80]$ suggesting that the coral protein homologue may have an additional role in 
Table 1 Regulatory proteins of symbiosis in the predicted proteome of A. digitifera

\begin{tabular}{|c|c|c|}
\hline Gene sequence & KEGG Orthology & Encoded protein description \\
\hline v1.06849 & K06110 & Exocyst complex component 3 \\
\hline v1.00063; v1.01826 & K06111 & Exocyst complex component 4 \\
\hline v1.06336; v1.06337; v1.15354 & K07195 & Exocyst complex component 7 \\
\hline v1.04340 [+ 4 other sequence copies] & K14966 & Host cell factor \\
\hline v1.01629; v1.19166 & K12481 & Rabenosyn-5 \\
\hline v1.18447 [+ 26 other sequence copies] & K07976 & Rab family, other (similar to Rab-6B) \\
\hline v1.02380 & $\mathrm{K} 12480$ & Rab GTPase-binding effector protein-1 \\
\hline v1.01032 & K13883 & Rab-interacting lysosomal protein \\
\hline v1.14682; v1.03256; v1.07709 & K12484 & Rab11 family-interacting protein-1/2/5 \\
\hline v1.13055; v1.13176; v1.16348 & K12485 & Rab11 family-interacting protein-3/4 \\
\hline V1.01275 & K07932 & Rab-like protein-2B \\
\hline v1.17629 [+ 13 other sequence copies] & K07933 & Rab-like protein-3 \\
\hline v1.03299; v1.09653 & K07934 & Rab-like protein-4 \\
\hline v1.08498 & K07935 & Rab-like protein-5 \\
\hline v1.16155 [+5 other sequence copies & K07874 & Ras-related protein Rab-1A \\
\hline v1.09098 & K07875 & Ras-related protein Rab-1B \\
\hline v1.13558; v1.08983 & K07877 & Ras-related protein Rab-2A \\
\hline v1.14260 & K07878 & Ras-related protein Rab-2B \\
\hline v1.07500; v1.20532; v1.07498 & K07884 & Ras-related protein Rab-3D \\
\hline v1.21242; v1.07502 & K07880 & Ras-related protein Rab- $4 \mathrm{~B}$ \\
\hline v1.01341; v1.05619 & K07888 & Ras-related protein Rab-5B \\
\hline v1.07125 & K07889 & Ras-related protein Rab-5C \\
\hline v1.09239 & K07893 & Ras-related protein Rab-6A \\
\hline v1.10443; v1.13335 & K07897 & Ras-related protein Rab-7A \\
\hline v1.03086; v1.17122; v1.07231 & K07916 & Ras-related protein Rab-7 L1 \\
\hline v1.02275 [+ 4 other sequence copies] & K07901 & Ras-related protein Rab-8A \\
\hline v1.24612 & K07899 & Ras-related protein Rab-9A \\
\hline v1.00411 & K07900 & Ras-related protein Rab-9B \\
\hline v1.10697; v1.01515 & K07903 & Ras-related protein Rab-10 \\
\hline v1.22278; v1.04408; v1.12528 & K07905 & Ras-related protein Rab-11B \\
\hline v1.07033; v1.23028 & K07881 & Ras-related protein Rab-14 \\
\hline v1.02275 & K07908 & Ras-related protein Rab-15 \\
\hline v1.16455; v1.14911; v1.14959 & K07910 & Ras-related protein Rab-18 \\
\hline v1.04714 & K07911 & Ras-related protein Rab-20 \\
\hline v1.01878; v1.12184 & K07890 & Ras-related protein Rab-21 \\
\hline v1.09930 & K06234 & Ras-related protein Rab-23 \\
\hline v1.13579; v1.12841 & K07912 & Ras-related protein Rab-24 \\
\hline v1.10183 & K07913 & Ras-related protein Rab-26 \\
\hline v1.08199 & K07885 & Ras-related protein Rab-27A \\
\hline v1.13978; v1.18893 & K07917 & Ras-related protein Rab-30 \\
\hline v1.03085; v1.06007; v1.07729 & K07918 & Ras-related protein Rab-32 \\
\hline v1.24721 & K07919 & Ras-related protein Rab-33A \\
\hline v1.18892 & K07920 & Ras-related protein Rab-33B \\
\hline v1.16060 & K07876 & Ras-related protein Rab-35 \\
\hline
\end{tabular}


Table 1 Regulatory proteins of symbiosis in the predicted proteome of A. digitifera (Continued)

\begin{tabular}{|c|c|c|}
\hline v1.15894 & K07922 & Ras-related protein Rab-36 \\
\hline v1.03080 & K07923 & Ras-related protein Rab-38 \\
\hline v1.21391 & K07924 & Ras-related protein Rab-39A \\
\hline v1.14786 & K07928 & Ras-related protein Rab-40 \\
\hline v1.05611 [+ 13 other sequence copies] & K08502 & Regulator of vacuolar morphogenesis (t-SNARE domain) \\
\hline v1.18253 & K08520 & SEC22 vesicle trafficking protein $A / C$ \\
\hline v1.15499 & K13814 & t-SNARE domain-containing protein 1 \\
\hline v1.05749 & K08516 & Synaptobrevin homologue YKT6 \\
\hline v1.13229 & K12768 & Synaptonemal complex protein ZIP1 \\
\hline v1.16533; v1.17141 & K08508 & Synaptosomal-associated protein, $23 \mathrm{kDa}$ \\
\hline v1.05301 & K08509 & Synaptosomal-associated protein, $29 \mathrm{kDa}$ \\
\hline v1.19071 & K04560 & Syntaxin $1 \mathrm{~A}$ \\
\hline v1.04614; v1.22747 & K08486 & Syntaxin 1B/2/3 \\
\hline v1.16462 & K08490 & Syntaxin 5 \\
\hline v1.20758; v1.21534 & K08498 & Syntaxin 6 \\
\hline v1.22836; v1.15499 & K08488 & Syntaxin 7 \\
\hline v1.01959; v1.24227 & K08501 & Syntaxin 8 \\
\hline v1.02007; v1.06683; v1.12727 & K08491 & Syntaxin 17 \\
\hline v1.21308; v1.11830; v1.01582 & K08492 & Syntaxin 18 \\
\hline v1.22100; v1.09457 & K08518 & Syntaxin binding protein 5 (tomosyn) \\
\hline v1.18555 & K08519 & Syntaxin binding protein 6 (amisyn) \\
\hline v1.12938 & K08500 & Syntaxin of plants SYP6 \\
\hline v1.06575 & K08506 & Syntaxin of plants SYP7 \\
\hline v1.14699 & K08507 & Unconventional SNARE in the endoplasmic reticulum protein 1 \\
\hline v1.23782 [+ 38 other sequence copies] & K08332 & Vacuolar protein 8 \\
\hline v1.15282; v1.24603; v1.01672 & K12196 & Vacuolar protein-sorting-associated protein 4 \\
\hline v1.17791 [+ 4 other sequence copies] & K12479 & Vacuolar protein sorting-associated protein 45 \\
\hline v1.20907 & K11664 & Vacuolar protein sorting-associated protein 72 \\
\hline v1.15996 [+ 5 other sequence copies] & K12199 & Vacuolar protein sorting-associated protein VTA1 \\
\hline v1.15614 & K08510 & Vesicle-associated membrane protein 1 (synaptobrevin) \\
\hline v1.13353 & K13504 & Vesicle-associated membrane protein 2 (synaptobrevin) \\
\hline v1.12458; v1.07528 & K13505 & Vesicle-associated membrane protein 3 (cellubrevin) \\
\hline v1.19735; v1.21831; v1.07186 & K08513 & Vesicle-associated membrane protein 4 (Golgi transport) \\
\hline V1.05299 & K08514 & Vesicle-associated membrane protein 5 (exocytosis) \\
\hline v1.13557; v1.24610 & K08515 & Vesicle-associated membrane protein 7 (exocytosis) \\
\hline v1.12279 & K08512 & Vesicle-associated membrane protein 8 (endobrevin) \\
\hline v1.00261; v1.08699; v1.04334 & K06096 & Vesicle-associated membrane protein A \\
\hline v1.20177 & K10707 & Vesicle-associated membrane protein B \\
\hline v1.15472; v1.03568 & K06027 & Vesicle-fusing ATPase \\
\hline v1.11431; v1.10487 & K08517 & Vesicle transport protein SEC22 \\
\hline v1.06393; v1.13003; v1.08735; v1.04261 & K08493 & Vesicle transport interaction with t-SNAREs 1 \\
\hline
\end{tabular}


Table 2 Distribution of Rab homologues of Aiptasia puchella, Hydra magnipapillata and Nematostella vectensis in the predicted proteome of $A$. digitifera

\begin{tabular}{|c|c|}
\hline A. digitifera Rab protein & $\begin{array}{l}\text { Cnidarian encoding } \\
\text { Rab homologue }\end{array}$ \\
\hline $\begin{array}{r}\text { Rab-like protein- 2B, Rab-2B Rab-3D, Rab-4B, } \\
\text { Rab-5B, Rab-26, Rab-32, Rab-38 }\end{array}$ & A. puchella \\
\hline Rab-like protein-3, Rab-36 & $N$. vectensis \\
\hline Rab-2A, Rab-23 & $\begin{array}{l}\text { A. puchella, } H \text {. } \\
\text { magnipapillata }\end{array}$ \\
\hline $\begin{array}{r}\text { Rab-like protein-6B, Rab-6A, Rab-7 L1, Rab-10, } \\
\text { Rab11B, Rab-30, Rab-33B }\end{array}$ & A. puchella, N. vectensis \\
\hline $\begin{array}{r}\text { Rab effector protein-1, Rab11-interacting } \\
\text { protein-3/4 }\end{array}$ & $\begin{array}{l}\text { H. magnipapillata, } \mathrm{N} \text {. } \\
\text { vectensis }\end{array}$ \\
\hline $\begin{array}{l}\text { Rab-like protein-4, Rab-like protein-5, Rab-1A, } \\
\text { Rab5C, Rab-7A, Rab-8A, Rab-9A, Rab-14, Rab- } \\
\text { 18, Rab-20, Rab-21, Rab-24, Rab-27A, Rab-35 }\end{array}$ & $\begin{array}{l}\text { A. puchella, } H \text {. } \\
\text { magnipapillata, } N \text {. } \\
\text { vectensis }\end{array}$ \\
\hline $\begin{array}{r}\text { Rab-interacting lysomal protein, Rab11- } \\
\text { interacting protein-1/2/5, Rab-1B, Rab-9B, } \\
\text { Rab-3A, Rab-39A, Rab-40 }\end{array}$ & No match \\
\hline
\end{tabular}

epigenetic reprogramming of the chromatin histoneDNA complex at different stages of development.

\section{Planula and early developmental proteins}

In this section we discuss predicted proteins encoded in the $A$. digitifera genome having functional homology to known proteins specific to early embryonic development, planula larvae function and morphogenesis, which are given in Table 4. Annotation of the coral genome reveals a large set of homeobox proteins involved in the regulation of anatomical development during morphogenesis. The homeobox is a highly conserved DNA sequence (homeodomain) within genes that binds to DNA in a sequence-specific manner [81] often at the promoter region of their target gene to affect transcription in the developing embryo. Amonst these transcriptional regulators, Hox genes are essential to metazoan development as their expressed proteins differentiate embryonic regions along the anterior-posterior axis (the Hox code) and are recognised for their contribution to the evolution of morphological diversity [82]. Hox genes are well characterised in cnidarians and, given their importance in embryonic development, it is not surprising that molecular evidence from the Cnidaria reveal that the genetic origins of Hox genes predate the cnidarian-bilaterian divergence [83-85] yet had evolved after divergence of the sponge and eumetazoan lineages [86]. Hox genes of cnidarians are typically located in a conserved genomic collinear cluster, which is apparent also for A. digitifera, whereby the order of the genes on the chromosome is the same as that of gene expression in the developing embryo. Included in our annotation are genes encoding
Table 3 Synaptotagmin proteins in the predicted proteome of $A$. digitifera

\begin{tabular}{|c|c|c|}
\hline Gene sequence & $\begin{array}{l}\text { GenBank } \\
\text { Accession }\end{array}$ & $\begin{array}{l}\text { Genome encoded } \\
\text { homologue }\end{array}$ \\
\hline v1.08623 & Gl:268530614 & $\begin{array}{l}\text { Caenorhabditis briggsae: } \\
\text { XP_002630433 (worm) }\end{array}$ \\
\hline $\begin{array}{l}\text { V1.20682; v1.10560; } \\
\text { v1.02080; v1.10015 }\end{array}$ & Gl:150416761 & $\begin{array}{l}\text { Platynereis dumerilii: } \\
\text { ABR68850 (worm) }\end{array}$ \\
\hline v1.10269; v1.04412 & Gl:288869516 & $\begin{array}{l}\text { Nasonia vitripennis: } \\
\text { NP_001165865 (wasp) }\end{array}$ \\
\hline v1.01508 & Gl:29378331 & $\begin{array}{l}\text { Lymnaea stagnalis: } \\
\text { AA093847 (snail) }\end{array}$ \\
\hline v1.18613 & Gl:391339919 & $\begin{array}{l}\text { Metaseiulus occidentalis: } \\
\text { XP_003744294 (mite) }\end{array}$ \\
\hline v1.07402 & Gl:260834895 & $\begin{array}{l}\text { Branchiostoma floridae: } \\
\text { XP_002612445 (lancelet) }\end{array}$ \\
\hline v1.01542 & Gl:149067023 & $\begin{array}{l}\text { Rattus norvegicus: EDM16756 } \\
\text { (rat) }\end{array}$ \\
\hline v1.20683 & Gl:383860584 & $\begin{array}{l}\text { Megachile rotundata: } \\
\text { XP_003705769 (bee) }\end{array}$ \\
\hline v1.17688 & Gl:48529130 & $\begin{array}{l}\text { Oreochromis niloticus; } \\
\text { XP_003452067 (fish) }\end{array}$ \\
\hline v1.15777; v1.14902 & Gl:269785031 & $\begin{array}{l}\text { Saccoglossus kowalevskii: } \\
\text { NP_001161667 (worm) }\end{array}$ \\
\hline v1.17175; v1.11521 & Gl:11559313 & $\begin{array}{l}\text { Halocynthia roretzi: } \\
\text { BAB18864 (ascidian) }\end{array}$ \\
\hline v.1.03344; v1.03345 & Gl:12658419 & $\begin{array}{l}\text { Manduca sexta; AF331039 } \\
\text { (moth) }\end{array}$ \\
\hline v1.16152 & Gl:395729192 & $\begin{array}{l}\text { Pongo abelii: XP_003780414 } \\
\text { (orangutan) }\end{array}$ \\
\hline v1.10268 & Gl:327283049 & $\begin{array}{l}\text { Anolis carolinensis: } \\
\text { XP_003226254 (lizard) }\end{array}$ \\
\hline v10.2778 & $\mathrm{Gl}: 125984480$ & $\begin{array}{l}\text { Drosophila pseudoobscura } \\
\text { XP_001356004.1 (fly) }\end{array}$ \\
\hline v1.02083; v1.02777 & $G \mid: 226490194$ & $\begin{array}{l}\text { Schistosoma japonicum: } \\
\text { CAX69339.1 (fluke) }\end{array}$ \\
\hline v1.04326 & Gl:167744962 & $\begin{array}{l}\text { Homo sapiens: 2R83_A } \\
\text { (human) }\end{array}$ \\
\hline v1.14682; v1.04180 & Gl:241704658 & $\begin{array}{l}\text { Ixodes scapularis: } \\
\text { XP_002411967 (tick) }\end{array}$ \\
\hline
\end{tabular}

two LIM homeobox proteins and a LIM homeobox transcription factor (Lhx) having conserved roles in neuronal development [87], which in $N$. vectensis are responsible for the development of neural networks in developing larvae and juvenile polyps [88]. Unlike $N$. vectensis [89], the coral genome expresses a homeobox BarH-like protein that in vertebrates directs neurogenesis [90]. Distinct from homeodomain proteins, but serving similar functions, are various protein activators, regulators and receptors of cellular morphogenesis. Annotation of the coral genome has revealed multiple sequence alignments to a protein homologue of the dishevelled-associated activator of 
Table 4 Planula and early developmental proteins in the predicted proteome of $\boldsymbol{A}$. digitifera

\begin{tabular}{|c|c|c|}
\hline Gene sequence & KEGG Orthology & Encoded protein description \\
\hline v1.09797; v1.11180; v1.08414 & K03776 & Aerotaxis receptor (oxygen sensing) \\
\hline v1.07838 [+5 other sequence copies] & K07822 & Archaeal flagellar protein FlaC \\
\hline v1.14039; v1.11310; v1.11309 & K05502 & Bone morphogenetic protein 1 \\
\hline v1.01025; v1.17008; v1.15796; v1.23658 & K04662 & Bone morphogenetic protein $2 / 4$ \\
\hline v1.02299; v1.07696; v1.10675 & K04663 & Bone morphogenetic protein 5/6/7/8 \\
\hline v1.06335; v1.01763 & K04673 & Bone morphogenetic protein receptor type- $1 \mathrm{~A}$ \\
\hline V1.13481 & K13578 & Bone morphogenetic protein receptor type-1B \\
\hline v1.10550 [+4 other sequence copies] & K04671 & Bone morphogenetic protein receptor type-2 \\
\hline v1.00912 [+4 other sequence copies] & K13579 & Bone morphogenetic protein receptor type- 1 , invertebrate \\
\hline v1.19370 & K14624 & C-C motif chemokine 2 \\
\hline v1.23163 & K12499 & C-C motif chemokine 5 \\
\hline v1.08576 & K05511 & C-C motif chemokine $15 / 23$ \\
\hline V1.09229 & K05512 & C-C motif chemokine 19/21 \\
\hline v1.09305 & K08373 & C-C chemokine receptor-like 2 \\
\hline v1.04942 & K04179 & C-C chemokine receptor type 4 \\
\hline v1.02658 & K04245 & Chemokine-like receptor 1 \\
\hline$\vee 1.21300$ & K12671 & C-X-C motif chemokine 10 \\
\hline v1.16396; v1.21991 & K10035 & C-X-C motif chemokine 16 \\
\hline v1.23712 & K11522 & Chemotaxis family two-component system response regulator PixG \\
\hline v1.09435 & K13490 & Chemotaxis family, histidine kinase sensor response regulator (WspE-like) \\
\hline v1.14142; v1.05300 & K05874 & Chemotaxis protein I, serine sensor receptor (MCP family) \\
\hline v1.07361 & K05877 & Chemotaxis protein IV, peptide sensor receptor (MCP family) \\
\hline v1.17411 & K03414 & Chemotaxis protein CheZ \\
\hline V1.16104 & K00575 & Chemotaxis protein methyltransferase Che R \\
\hline v1.15537 [+ 7 other sequence copies] & K08482 & Circadian clock protein KaiC \\
\hline v1.14925 [+ 4 other sequence copies] & K02223 & Circadian locomoter output cycles kaput protein \\
\hline v1.06432 [+ 9 other sequence copies] & K04512 & Dishevelled associated activator of morphogenesis \\
\hline v1.17637 [+ 70 other sequence copies] & K10408 & Dynein heavy chain, axonemal \\
\hline v1.00202 [+5 other sequence copies] & K10409 & Dynein intermediate chain 1, axonemal \\
\hline v1.04986; v1.09649; v1.23645 & K11143 & Dynein intermediate chain 2, axonemal \\
\hline v1.08695; v1.09481; v1.23153 & K10411 & Dynein light chain 1, axonemal \\
\hline v1.11684 & K10412 & Dynein light chain 4, axonemal \\
\hline v1.23322; v1.01131; v1.04207 & K10410 & Dynein light intermediate chain, axonemal \\
\hline V1.14083 & K02401 & Flagellar biosynthetic protein FlhB \\
\hline v1.16997 & K02420 & Flagellar biosynthetic protein FliQ \\
\hline v1.02867 & K02396 & Flagellar hook-associated protein 1 FlgK \\
\hline v1.18101; v1.13427 & K02408 & Flagellar hook-basal body complex protein FliE \\
\hline v1.04339; v1.07633 & K06603 & Flagellar protein FlaG \\
\hline v1.17895[+5 other sequence copies] & K02383 & Flagellar protein FlbB \\
\hline v1.21111 & K02413 & Flagellar protein FliJ \\
\hline v1.17651 [+ 13 other sequence copies] & K02415 & Flagellar protein FliL \\
\hline v1.01971 [+ 6 other sequence copies] & K02418 & Flagellar protein $\mathrm{FliO} / \mathrm{FliZ}$ \\
\hline v1.14031 & K02423 & Flagellar protein FliT \\
\hline v1.08025 & K02394 & Flagellar P-ring protein precursor Flgl \\
\hline
\end{tabular}


Table 4 Planula and early developmental proteins in the predicted proteome of $\boldsymbol{A}$. digitifera (Continued)

\begin{tabular}{|c|c|c|}
\hline v1.02396; v1.15777 & K02409 & Flagellar M-ring protein FliF \\
\hline v1.20693 & K09451 & Homeobox protein aristaless-like 4 \\
\hline v1.24732 [+5 other sequence copies] & K09452 & Homeobox protein aristaless-related \\
\hline v1.15788; v1.19334; v1.04164 & K09313 & Homeobox protein cut-like \\
\hline v1.01801 & K09319 & Homeobox protein engrailed \\
\hline v1.16835; v1.06323 & K09320 & Homeobox even-skipped homologue protein \\
\hline v1.0412; v1.054771 & K09354 & Homeobox protein expressed in ES cells 1 \\
\hline v1.13604 & K09324 & Homeobox protein goosecoid \\
\hline v1.06346; v1.08163 & K09325 & Homeobox protein goosecoid-like \\
\hline v1.17295; v1.17294 & K09361 & Homeobox protein, BarH-like (vertebrate neurogenesis) \\
\hline v1.07457 & K09316 & Homeobox protein DLX, invertebrate \\
\hline v1.11157; v1.08573; v1.15250 & K09317 & Homeobox protein EMX \\
\hline v1.01800 & K09321 & Homeobox protein GBX \\
\hline v1.10929; v1.06346; v1.05443; v1.07458 & K09310 & Homeobox protein GSH \\
\hline v1.13684; v1.24444 & K08025 & Homeobox protein HB9 \\
\hline v1.16254; v1.16064 & K08024 & Homeobox protein HEX \\
\hline v1.07458; v1.06706; v1.06705 & K09339 & Homeobox protein HLX1 \\
\hline v1.06347; v1.06348; v1.17294 & K09302 & Homeobox protein HoxA/B2 \\
\hline v1.06125 & K09306 & Homeobox protein HoxA/B/C6 \\
\hline V1.19818 & K09304 & Homeobox protein HoxA/B/C/D4 \\
\hline v1.06706 & K09301 & Homeobox protein HoxA/B/D1 \\
\hline v1.02056 & K09353 & Homeobox protein LBX \\
\hline v1.06347; v1.06348 & K09328 & Homeobox protein Unc-4 \\
\hline v1.24342; v1.04552 & K09318 & Homeobox protein ventral anterior \\
\hline v1.03823; v1.10070; v1.04435 & K09309 & Homeobox protein Nkx-1 \\
\hline v1.12852 [+ 4 other sequence copies] & K08029 & Homeobox protein Nkx-2.2 \\
\hline v1.21630 & K09345 & Homeobox protein Nkx-2.5 \\
\hline v1.10625 & K09347 & Homeobox protein Nkx-2.8 \\
\hline v1.10625; v1.13865; v1.05476 & K09348 & Homeobox protein Nkx-3.1 \\
\hline v1.21628; v1.05475; v1.05477 & K09995 & Homeobox protein Nkx-3.2 \\
\hline v1.06135; v1.10071 & K09349 & Homeobox protein Nkx-5 \\
\hline v1.14702 & K08030 & Homeobox protein Nkx-6.1 \\
\hline v1.14917; v1.11907 & K09350 & Homeobox protein Nkx-6.2 \\
\hline v1.00777; v1.21453 & K09322 & Homeobox protein MOX \\
\hline v1.00602 [+ 6 other sequence copies] & K09326 & Homeobox protein OTX \\
\hline V1.16722; v1.12785 & K09374 & LIM homeobox protein $3 / 4$ \\
\hline v1.11281; v1.05135 & K09375 & LIM homeobox protein $6 / 8$ \\
\hline v1.07988; v1.22037 & K09371 & LIM homeobox transcription factor 1 \\
\hline v1.09328 [+ 5 other sequence copies] & K10394 & Kinesin family member $3 / 17$ \\
\hline v1.09196; v1.12479 & K11525 & Methyl-accepting chemotaxis protein PixJ (MCP family) \\
\hline v1.17028; v1.13473 & K08473 & Nematode chemoreceptor \\
\hline v1.13159; v1.00655 & K09330 & Paired mesoderm homeobox protein 2 \\
\hline v1.15178; v1.10962; v1.16587; v1.01557 & K02633 & Period circadian protein \\
\hline v1.23288; v1.13857 & K04627 & Pheromone a factor receptor \\
\hline v1.22464; v1.17135 & K11213 & Pheromone alpha factor receptor \\
\hline
\end{tabular}


Table 4 Planula and early developmental proteins in the predicted proteome of $\boldsymbol{A}$. digitifera (Continued)

\begin{tabular}{rll}
\hline 1.05611 [+ 13 other sequence copies] & K08502 & Regulator of vacuolar morphogenesis \\
v1.04431 & K09333 & Retina and anterior neural fold homeobox-like protein \\
v1.17636 & K09331 & Short stature homeobox protein \\
v1.14704 & K09340 & T-cell leukemia homeobox protein \\
v1.11765 & $N A^{\mathbf{1}}$ & Tektin \\
v1.04154 & K02669 & Twitching motility protein PilT \\
\hline
\end{tabular}

${ }^{1} \mathrm{NA} \mathrm{KEGG}$ orthology designation not assigned.

morphogenesis 1 (Daam1) that initiates cytoskeleton formation via the control of actin assembly. Daam1 was found crucial for gastrulation in Xenopus [91], wherein Daam1 mutants of Drosophilia exhibit trachea defects [92], and in mammals Daam1 is highly expressed in multiple developing organs and is deemed essential for cardiac morphogenesis [93]. Similar morphogenetic genes express regulatory proteins that are necessary for vacuole biogenesis in yeasts [94]. Others express bone morphogenetic proteins (and their BMP receptors), which are potent multi-functional growth activators that belong to the transforming growth factor beta (TGFbeta) cytokine superfamily of proteins that in humans have various functions during embryogenesis, skeletal formation, neurogenesis and haematopoiesis [95]. However, since many of the homeobox and morgenetic proteins (Table 4) are homologues of proteins with functions ascribed to higher organisms, their precise function in $A$. digitifera cannot be ascertained by KEGG orthology alone.

Another protein encoded in the $A$. digitifera genome is a retina and anterior neural fold homeobox-like (RAX) protein that may activate the development of primitive coral photoreceptors [96,97], including a blue light-sensing, cryptochrome photoreceptor that in $A$. millepora is implicated in the detection of light from the lunar cycle of night time illumination to signal synchronous coral spawning $[98,99]$. Photosensitive behaviours and the circadian rhythms of corals are well described, and diurnal cycles of gene transcription that regulate circadian biological processes in the coral $A$. millepora have been reported [100]. Such traits in A. millepora appear regulated by an endogenous biological clock entrained to daily cycles of solar illumination [101]. Annotation of the $A$. digitifera genome reveals a circadian timekeeper protein KaiC [102] that in cyanobacteria is activated during the diurnal phosphorylation rhythm [103,104]. In Synechococcus elongatus, KaiC regulates the rhythmic expression of all other proteins encoded in the genome [105], yet no homologue of any of the prokaryotic clustered circadian kia $A B C$ genes has been identified in eukaryotes [106]. In Drosophila, KaiC together with a homologue of the eukaryotic period (Per) circadian protein drives circadian rhythms in eclosion (hatching) and locomotor activity [107]. Nevertheless, a circadian locomotor output cycles kaput (CLOCK) homologue (Table 4) was found in our annotation. Since CLOCK proteins serve as an essential activator of downstream elements in pathways critical to the regulation of circadian rhythms in eukaryotes [108], it would be worthy to examine how transcription of the RAX-like homeobox protein in this coral contributes to the development of circadian functions by activation of kaiC, per and Clock genes. Such a study might reveal that components of the animal circadian clock are more ancient than data previously suggested [109].

Broadcast-spawning corals, such as A. digitifera, release gametes, and the fertilised eggs develop into planula larvae within the water column until they have reached settlement competency, find a suitable hard substrate, attach and develop into the polyp on metamorphosis. Coral sperm and planula larvae achieve motility using flagella (sperm) or cilia (larvae) as their locomotor organelles. The eukaryotic axonemal proteins of cilia and flagella are composed of a dynein ATPase protein to provide mechanochemical energy transduction together with the principle structural proteins of the ciliary/flagellar microtubules [110]. The flagellar/ciliary microtubules consist of filaments composed of $\alpha$ - and $\beta$-tubulins, microtubule-stabilising tektins and kinesin motor proteins [111-113]. The coral genome encodes members of the dynein axonemal (flagella and cilia) proteins (Table 4) and many of the dynein cytoplasmic proteins (not tabulated), the latter being involved in intracellular organelle transport and centrosome assembly. The coral genome encodes $\alpha$ - and $\beta$-tubulins and members of the eukaryotic kinesin superfamily proteins (not tabulated). Amongst the many kinesin proteins encoded in the coral genome is the kinesin family member $3 / 17$ protein, which is a direct homologue of the kinesin-II intraflagellar transport protein FLA10 essential for flagella assembly in the alga Chlamydomonas [114]. The microtubule-stabilising tektin protein, which is required for cilia and flagella assembly [113], is also encoded in the coral genome [note: there is no KEGG orthology profile assigned to this protein]. It was a surprise, however, to find a large complement of 
prokaryotic flagellar proteins encoded in the coral genome consisting of archaeal flagellar ( $\mathrm{FlaC}$ and $\mathrm{FlaG}$ ), bacterial filament (FibB, FlliE, FliF, FliJ, FliK, FliO/FliZ, FliQ and FliT) homologue components (Table 4). Included also are the prokaryote homologues FlgN and FlbB that regulate transcriptional activation of flagellar assembly $[115,116]$ and FlhB which controls the substrate specificity of the entire prokaryotic flagellar apparatus [117]. Encoded in the coral genome is a flagella-independent Type IV twitching mobility protein PilT that affords social gliding translocation in many prokaryotic organisms controlled by complex signal transduction systems that include two-component sensor regulators [118]. It is unlikely that these genes are derived from contamination from bacterial DNA. Such contamination would manifest itself by the random occurrence of bacterial genes from the whole genome including many housekeeping genes. In this case, the genes occur as members of groups with specialised functions, suggesting that multiple horizontal gene transfers between bacteria and the coral genome have occurred [119]. Their precise function in A. digitifera remains unknown; homologues of these prokaryotic genes have not been described previously in any other eukaryote genome.

Linked closely with flagellar/ciliary proteins are the sensory receptors that signal chemoattraction or avoidance to direct cellular motility. The coral genome reveals a variety of genes that encode chemoreceptor and chemotaxis proteins (Table 4). The chemoreceptor proteins of $A$. digitifera include an oxygen-sensing aerotaxis receptor that in bacteria invokes an avoidance response to anoxic micro-environments [120]. Encoded also are a nematode sensory chemoreceptor homologue [121], two homologous pheromone factor receptor proteins that in fungi activate a species-specific mating response [122], three chemotaxis protein sensor receptors belonging to the methyl-accepting chemotaxis family of proteins (MCPs) in bacteria and archaea [123], and two proteins (CheZ and CheR) and two regulators (PixG and WspE) of the twocomponent signal transduction (TCST) system for activation of gene expression. In bacteria and archea, as well as some plants, fungi and protozoa [124], TCST systems mediate many cellular processes that respond to a broad range of environmental stimuli via activation of a specific histidine (or serine) kinase sensor and its cognate response regulator [125]. There are 77 sequence matches to various elements of the TCST family of proteins in the $A$ digitifera genome (data not tabulated). Included also are genes encoding members of the chemotactic cytokine (chemokine) family of sensory proteins that on secretion directs chemotaxis in nearby responsive cells by stimulating target chemokine receptors; both chemokine and chemokine receptor proteins are encoded in the coral genome. Significantly, sensory chemokines/chemokine receptors are found in all vertebrates, some viruses and some groups of bacteria, but none have been described previously for invertebrates [126].

\section{Neural messengers, receptors and sensory proteins}

Corals and other cnidarians are the earliest extant group of organisms to have a primitive nervous system network [127] thought to be evolved from a eumetazoan ancestor prior to the divergence of Cnidaria and the Bilateria [128,129]. Unlike marine sponges (Porifera) that predate synaptic innovation [130], cnidarians possess a homogenous nerve net that, although lacking any form of cephalization, accommodates fundamental neurosensory transmission across the nerve net to end in a motoneural junction to coordinate tentacle movement required for feeding and predator avoidance [131]. The nervous systems of cnidarians consist of both ectodermal sensory cells and their effector cells and endodermal multipolar ganglions capable of neurotransmission [132]. At the functional level, synaptic transmission in cnidarians relies on fast neurotransmitters (glutamate, GABA, glycine) and slow neurotransmitters (catecholamine, serotonin, neuropeptides) for sensory-signal conduction [133]. At the ultrastructural level, many cnidarian neurons have multifunctional traits of sensory, neurosecretory and stimulatory attributes [134]. Significantly, the genome of $A$. digitifera encodes the expression of a ciliary neurotrophic factor, which is a polypeptide hormone and nerve growth factor that promotes neurotransmitter synthesis, neurite outgrowth and regeneration [135]. Additionally, the coral genome encodes nerve growth factor and neurotrophic kinase receptors, a survival motor neuron protein, a survival neuron splicing factor, the neural outgrowth protein neurotrimin, and a neurotrophin growth factor attributed to signalling neuron survival, differentiation and growth (Table 5). Encoded for neuron regulation and development are several neuron cation-gated channels, a neuronal guanine nucleotide exchange factor, a neurotransmitter $\mathrm{Na}^{+}$ symporter, several neurogenic differentiation proteins, a neuronal PAS domain transcription factor for activation of neurogenesis, the axon guidance protein neurophilin-2, a neural crest protein of embryonic neural development, neural ELAV-like transcription proteins of neurogenesis, a Notch protein (79 sequence domain matches) and a neutralized protein subset of the Notch signalling pathway that promotes neuron proliferation in early neurogenic development. Structural elements of the coral nerve net include neurofilament polypeptides and neuronal adhesion proteins.

Cnidarians differentiate highly specialised sensory and mechanoreceptor cells involved in the capture of prey and for defence against predators. Their stinging cells, termed nematocysts or cnidocytes, are stimulated by adjacent chemosensory cells. Nematocysts trigger the release of a stinging barb (cnidae tubule) via ultra-fast 
exocytosis on physical contact with ciliary mechanoreceptors of the cnidocyte to deliver the discharge of its venom [136]. Despite considerable advances in the sensory biology of cnidarians, knowledge of the specific receptor genes that regulate cnidocyte function remains incomplete. In Hydra, and perhaps other cnidarians, cnidocyte discharge is controlled by an ancient lightactivated, opsin-mediated phototransduction pathway [137] that precedes the evolution of cubozoan (box jellyfish) eyes [138]; cubozoans are the most basal of animals to have eyes containing a lens and ciliary-type visual cells similar to that of vertebrate eyes [139]. These G-coupled opsin photoreceptors of the retinylidene-forming protein family encoded in the genome of $A$. digitifera include rhodopsin, bacteriorhodopsin, c-opsin, r-opsin and $\mathrm{G}_{0^{-}}$ opsin (Table 5), but not the Gs-subfamily of opsin receptors reported to be present in sea anemones, hydra and jellyfish [140], that together with cyclic nucleotide-gated $(\mathrm{CNG})$ ion channel proteins, arrestin ( $\beta$-adrenergic receptor inhibitor) and other retino-protein receptors, are usual components of the bilaterian phototransduction cascade. Present also are genes to express rhodopsin kinase and $\beta$ adrenergic receptor kinase which are related members of the serine/threonine kinase family of proteins that specifically initiate deactivation of G-protein coupled receptors. Additional proteins of retinol metabolism of the phototransduction pathway encoded in the $A$. digitifera genome are retinol dehydrogenase, all-trans-retinol 13,14 reductase and phosphatidylcholine (lichthin)-retinol O-acyltransferase, a neural retina-specific leucine zipper protein that is an intrinsic regulator of photoreceptor development and function, and a retina and anterior neural fold homeobox-like protein that modulates the expression of photoreceptor genes within the rhodopsin promoter. The genome of $A$. digitifera encodes also a blue light-sensing, cryptochrome photoreceptor thought to signal synchronous coral spawning by detecting illumination from the lunar cycle $[98,99]$.

The $A$. digitifera genome reveals genes to express a broad array of neurotransmitter receptor proteins (Table 5), including glycine and glutamate neuroreceptors, adrenergic receptors that target non-dopamine catecholamines (i.e., epinephrine and norepinephrine), dopamine, muscarinic and nicotinic acetylcholine receptors, sensory G proteincoupled receptors and $\gamma$-aminobutyric acid (GABA) ligand-gated ion channel and $G$ protein-coupled receptors (and inhibitors), several of which are encoded in high copy numbers. Cellular trafficking of neurotransmitters to presynaptic terminals is essential for neurotransmission, and significantly the genome of A. digitifera encodes a wide range of solute carrier neurotransmitter transporters, including a high affinity choline transporter and an acetylcholine-specific protein belonging to the major facilitator superfamily (MFS) of secondary transporters. Encoded also is dopamine $\beta$-monooxygenase that catalyses the conversion of dopamine to norepinephrine in the catecholamine biosynthetic pathway, which is necessary for cross-activation of adrenergic neuroreceptors [141]. Notably, the A. digitifera genome encodes acetylcholinesterase that is expressed at neuromuscular junctions and cholinergic synapses where its protease activity serves to terminate synaptic transmission.

The primitive nervous networks of cnidarians are strongly peptidergic with at least 35 neuropeptides identified from different cnidarian classes [142]. Our annotation of the sequenced $A$. digitifera genome, however, revealed only the neuropeptide FF-amide neurotransmitter, a RF amide related peptide, and its neuropeptide FF and Y receptors (Table 5). Neuropeptides are usually expressed as large precursor proteins which comprise multiple copies of "immature" neuropeptides. Our annotation did not readily reveal these precursor neuropeptide proteins, but we did find enzymes required for their processing, for example, a variety of carboxypeptidase enzymes (not tabulated) that remove propeptide carboxyl residues at basic peptidase sites, and the mature peptide neurotransmitters that are finished by consecutive modification by peptidylglycine ( $\alpha$-hydroxylating) monooxidase (PHM) and peptidyl $\alpha$-hydroxyglycine $\alpha$-amidating lyase (PAL) enzymes, both of which are commonly expressed in mammals as a single bifunctional peptidylglycine monooxy genase (K00504/EC 1.14.17.3) [143]. Our extensive catalogue of animal-like neural and sensory proteins revealed by genome annotation is testament that essential neurobiological features were developed in the primitive neural networks of early eumetazoan evolution.

\section{Calcification and $\mathrm{Ca}^{2+}$-signalling proteins}

The massive structures of coral reefs evident today are a construction of aggregated calcium carbonate deposited over long geological time by scleractinian corals and other calcifying organisms, yet our understanding of the molecular processes that regulate the biological processes of coral calcification is limited [144]. $\mathrm{Ca}^{2+}$ transfer from seawater to the calicoblastic site of coral calcification occurs by passive diffusion through the gastrovascular cavity [145] and by active calcium transport [146]. Active entry of $\mathrm{Ca}^{2+}$ through the oral epithelial layer is regulated by voltage-dependent calcium channels, such as demonstrated by the L-type alpha protein cloned from the reef-building coral Stylophora pistillata [147]. $\mathrm{Ca}^{2+}$ transport across the calioblastic ectoderm to the extracellular calcifying site is facilitated by the plasmamembrane ATP-dependent calcium pump that in $S$. pistillata resemble the $\mathrm{Ca}^{2+}$-ATPase family of mammalian proteins [148]. By $2 \mathrm{H}^{+} / \mathrm{Ca}^{2+}$-exchange at the calioblastic membrane, $\mathrm{Ca}^{2+}$-ATPase removes $\mathrm{H}^{+}$(from the net reaction $\mathrm{Ca}^{2+}+\mathrm{CO}_{2}+\mathrm{H}_{2} \mathrm{O} \Rightarrow \mathrm{CaCO}_{3}+2 \mathrm{H}^{+}$) thereby 
Table 5 Neuronal and sensory proteins in the predicted proteome of A. digitifera

\begin{tabular}{|c|c|c|}
\hline Gene sequence & KEGG Orthology & Encoded protein description \\
\hline v1.01918 [+ 5 other sequence copies] & K01049 & Acetylcholinesterase \\
\hline v1.18087; v1.14516 & K04136 & Adrenergic receptor alpha-1B \\
\hline v1.06394 & K04137 & Adrenergic receptor alpha-1D \\
\hline v1.09628; v1.15688; v1.00966 & K04140 & Adrenergic receptor alpha- $2 \mathrm{C}$ \\
\hline v1.19831; v1.20450 & K04142 & Adrenergic receptor beta-2 \\
\hline v.17293 & K00910 & beta-Adrenergic-receptor kinase \\
\hline v1.13740 [+ 5 other sequence copies $]$ & K04828 & Amiloride-sensitive cation channel 1, neuronal (degenerin) \\
\hline v1.23541 [+ 6 other sequence copies] & K04829 & Amiloride-sensitive cation channel 2, neuronal \\
\hline v1.09323 [+ 4 other sequence copies] & K04439 & beta-Arrestin \\
\hline v1.07723; v1.22465 & K04641 & Bacteriorhodopsin \\
\hline v1.08062 & K05420 & Ciliary neurotrophic factor \\
\hline v1.03288 [+ 5 other sequence copies] & K02295 & Cryptochrome \\
\hline v1.20011; v1.20036; v1.20084; v1.18607 & K04948 & Cyclic nucleotide gated channel alpha 1 \\
\hline$\vee 1.21470$ & K04951 & Cyclic nucleotide gated channel alpha 4 \\
\hline v1.21783; v1.01466; v1.01466; v1.01466 & K05326 & Cyclic nucleotide gated channel, invertebrate \\
\hline v1.03645 & K05391 & Cyclic nucleotide gated channel, other eukaryote \\
\hline$\vee 1.21256$ & K08762 & Diazepam-binding inhibitor (GABA receptor, acyl-CoA-binding protein) \\
\hline v1.22156 [+ 6 other sequence copies] & K00503 & Dopamine beta-monooxygenase \\
\hline v1.21775: v1.15989 & K04148 & Dopamine D1-like receptor \\
\hline v1.14160; v1.01697 & K04144 & Dopamine receptor D1 \\
\hline v1.05089; v1.20018 & K04145 & Dopamine receptor D2 \\
\hline v1.14030; v1.23273 & K04146 & Dopamine receptor D3 \\
\hline v1.20536 & K13088 & ELAV-like protein 1 \\
\hline v1.18658 [+ 5 other sequence copies] & K13208 & ELAV-like protein 2/3/4 \\
\hline v1.05774 [+ 18 other sequence copies] & K04313 & G protein-coupled receptor 6 \\
\hline v1.00572; v1.18152 & K08404 & G protein-coupled receptor 17 \\
\hline v1.23842 & K04316 & G protein-coupled receptor 19 \\
\hline v1.03948 & K08411 & G protein-coupled receptor 26 \\
\hline v1.09271 & K08383 & G protein-coupled receptor 34 \\
\hline v1.05595 & K04243 & G protein-coupled receptor 37 (endothelin receptor type B-like) \\
\hline v1.04019 & K08409 & G protein-coupled receptor 45 \\
\hline v1.19913; v1.09821; v1.04291 & K08450 & G protein-coupled receptor 56 \\
\hline v1.05404 & K04321 & G protein-coupled receptor 63 \\
\hline v1.02179; v1.10397 & K08451 & G protein-coupled receptor 64 \\
\hline v1.23269 [+ 5 other sequence copies] & K08408 & G protein-coupled receptor 68 \\
\hline v1.21091 & K08421 & G protein-coupled receptor 84 \\
\hline v1.11008 & K04302 & G protein-coupled receptor 85 \\
\hline v1.21884; v1.01951 & K08452 & G protein-coupled receptor 97 \\
\hline v1.03243 [+ 13 other sequence copies] & K08378 & G protein-coupled receptor 103 \\
\hline v1.13790; v1.18939 & K08453 & G protein-coupled receptor 110 \\
\hline v1.09442; v1.14019 & K08455 & G protein-coupled receptor 112 \\
\hline v1.24009 & K08456 & G protein-coupled receptor 113 \\
\hline v1.04290 & K08459 & G protein-coupled receptor 114 \\
\hline v1.06608; v1.24223 & K08457 & G protein-coupled receptor 115 \\
\hline
\end{tabular}


Table $\mathbf{5}$ Neuronal and sensory proteins in the predicted proteome of $\boldsymbol{A}$. digitifera (Continued)

\begin{tabular}{|c|c|c|}
\hline v1.10800 [+ 6 other sequence copies] & K08458 & G protein-coupled receptor 116 \\
\hline v1.07662 [+ 6 other sequence copies] & K08462 & G protein-coupled receptor 125 \\
\hline v1.09663; v1.08981 & K08463 & G protein-coupled receptor 126 \\
\hline v1.24252 & K08464 & G protein-coupled receptor 128 \\
\hline v1.02750 [+ 26 other sequence copies] & K08465 & G protein-coupled receptor 133 \\
\hline v1.05774 [+ 11 other sequence copies] & K08466 & G protein-coupled receptor 144 \\
\hline v1.05497; v1.13272; v1.01323 & K08436 & G protein-coupled receptor 152 \\
\hline v1.08653 [+ 5 other sequence copies] & K08467 & G protein-coupled receptor 157 \\
\hline v1.11807; v1.10392; v1.10394 & K08469 & G protein-coupled receptor 158 \\
\hline v1.07294; v1.00247 & K08439 & G protein-coupled receptor 161 \\
\hline v1.05167 & K08442 & G protein-coupled receptor 176 \\
\hline v1.08677; v1.23465; v1.19865; v1.06986 & K12762 & G protein-coupled receptor GPR1 \\
\hline v1.13395 & K08291 & G protein-coupled receptor kinase \\
\hline v1.18529; v1.07599; v1.05558 & K12487 & G protein-coupled receptor kinase interactor 2 \\
\hline v1.02481 & K04619 & G protein-coupled receptor family C group 5 member B \\
\hline v1.22242 & K04622 & G protein-coupled receptor family $C$ group 6 member A \\
\hline v1.08625; v1.13650; v1.13048; v1.18694 & K04599 & G protein-coupled receptor Mth (Methuselah protein) \\
\hline v1.07465; v1.10540 & K08341 & GABA(A) receptor-associated protein (autophagy-related protein 8) \\
\hline v1.09831 [+ 30 other sequence copies] & K05270 & Gamma-aminobutyric acid (GABA) receptor, invertebrate \\
\hline v1.18702; v1.11701 & K05183 & Gamma-aminobutyric acid (GABA) A receptor beta-3 \\
\hline v1.04252 [+ 6 other sequence copies] & K05185 & Gamma-aminobutyric acid (GABA) A receptor epsilon \\
\hline v1.06325 & K05186 & Gamma-aminobutyric acid (GABA) A receptor gamma-1 \\
\hline v1.00048 & K05188 & Gamma-aminobutyric acid (GABA) A receptor gamma-3 \\
\hline v1.07506 [+ 6 other sequence copies] & K04615 & Gamma-aminobutyric acid (GABA) B receptor 1 \\
\hline v1.07506 [+ 24 other sequence copies] & K04616 & Gamma-aminobutyric acid (GABA) B receptor 2 \\
\hline v1.06426; v1.10563; v1.01138 & K05192 & Gamma-aminobutyric acid (GABA) receptor theta \\
\hline v1.15485 & K05198 & Glutamate receptor, ionotropic, AMPA 2 \\
\hline v1.09807 & K05200 & Glutamate receptor, ionotropic, AMPA 4 \\
\hline v1.04764 & K05207 & Glutamate receptor, ionotropic, delta 2 \\
\hline v1.15247 [+ 12 other sequence copies] & K05313 & Glutamate receptor, ionotropic, invertebrate \\
\hline v1.15247 [+ 7 other sequence copies] & K05202 & Glutamate receptor, ionotropic, kainate 2 \\
\hline v1.00617 & K05203 & Glutamate receptor, ionotropic, kainate 3 \\
\hline v1.09688 [+ 6 other sequence copies] & K05208 & Glutamate receptor, ionotropic, N-methyl D-aspartate 1 \\
\hline v1.21204 [+ 4 other sequence copies] & K05212 & Glutamate receptor, ionotropic, N-methyl-D-aspartate 2D \\
\hline v1.01622 & K05214 & Glutamate receptor, ionotropic, N-methyl-D-aspartate 3B \\
\hline v1.01418 [+ 5 other sequence copies] & K05387 & Glutamate receptor, ionotropic, other eukaryote \\
\hline v1.04275 & K05194 & Glycine receptor alpha-2 \\
\hline v1.10737; v1.06885 & K05195 & Glycine receptor alpha-3 \\
\hline v1.05488 & K05271 & Glycine receptor alpha-4 \\
\hline v1.08900; v1.06885 & K05196 & Glycine receptor beta \\
\hline v1.18634 & K05397 & Glycine receptor, invertebrate \\
\hline v1.14569; v1.14570 & K09071 & Heart-and neural crest derivatives-expressed protein \\
\hline v1.16783 [+ 4 other sequence copies] & K02168 & High-affinity choline transport protein \\
\hline V1.13837 & K07608 & Internexin neuronal intermediate filament protein, alpha \\
\hline v1.01671 & K04309 & Leucine-rich repeat-containing $\mathrm{G}$ protein-coupled receptor 4 \\
\hline
\end{tabular}


Table $\mathbf{5}$ Neuronal and sensory proteins in the predicted proteome of $\boldsymbol{A}$. digitifera (Continued)

\begin{tabular}{|c|c|c|}
\hline v1.09480; v1.05605 & K04308 & Leucine-rich repeat-containing G protein-coupled receptor 5 \\
\hline v1.15300 [+ 8 other sequence copies] & K08399 & Leucine-rich repeat-containing G protein-coupled receptor 6 \\
\hline v1.17524 [+ 14 other sequence copies] & K04306 & Leucine-rich repeat-containing G protein-coupled receptor 7 \\
\hline v1.21700; v1.03578; v1.17196 & K04307 & Leucine-rich repeat-containing G protein-coupled receptor 8 \\
\hline v1.16104 & K08396 & Mas-related $\mathrm{G}$ protein-coupled receptor member $X$ \\
\hline v1.08718; v1.02042; v1.02042 & K04604 & Metabotropic glutamate receptor $1 / 5$ \\
\hline v1.22794 [+ 7 other sequence copies] & K04605 & Metabotropic glutamate receptor $2 / 3$ \\
\hline v1.15331 & K04607 & Metabotropic glutamate receptor 4 \\
\hline v1.01418 & K04608 & Metabotropic glutamate receptor $6 / 7 / 8$ \\
\hline v1.21698; v1.04544; v1.21739 & K14636 & MFS transporter, solute carrier family 18 (acetylcholine transporter) 3 \\
\hline v1.05751; v1.19720; v1.22165; v1.02336 & K04134 & Muscarinic acetylcholine receptor \\
\hline v1.11550 & K04129 & Muscarinic acetylcholine receptor M1 \\
\hline v1.01913 [+ 4 other sequence copies] & K04131 & Muscarinic acetylcholine receptor M3 \\
\hline v1.18723 & K04132 & Muscarinic acetylcholine receptor M4 \\
\hline v1.08171 & K04133 & Muscarinic acetylcholine receptor M5 \\
\hline v1.07408 [+ 34 other sequence copies] & K02583 & Nerve growth factor receptor (TNFR superfamily member 16) \\
\hline v1.15265 [+ 91 other sequence copies] & K06491 & Neural cell adhesion molecule \\
\hline v1.13789; v1.24010; v1.03980 & K09038 & Neural retina-specific leucine zipper protein \\
\hline v1.24586; v1.16386; v1.16387 & K08052 & Neurofibromin 1 \\
\hline v1.05520; v1.15407; v1.07950 & K04572 & Neurofilament light polypeptide \\
\hline v1.19724 & K04573 & Neurofilament medium polypeptide (neurofilament 3) \\
\hline v1.15787 [+ 4 other sequence copies] & K09081 & Neurogenin 1 (neurogenic differentiation protein) \\
\hline v1.00345; v1.05338; v1.10997 & K08033 & Neurogenic differentiation factor 1 \\
\hline v1.07355; v1.14517 & K09078 & Neurogenic differentiation factor 2 \\
\hline v1.08832 & K09079 & Neurogenic differentiation factor 4 \\
\hline v1.06678; v1.06677 & K01393 & Neurolysin \\
\hline v1.16238 [+ 19 other sequence copies] & K06756 & Neuronal cell adhesion molecule \\
\hline v1.20460; v1.16967 & K06757 & Neurofascin NFASC (cell adhesion molecule CAMs) \\
\hline v1.22060; v1.03561 & K07525 & Neuronal guanine nucleotide exchange factor \\
\hline V1.03908 & K09098 & Neuronal PAS domain-containing protein $1 / 3$ \\
\hline v1.00089 & K05247 & Neuropeptide FF-amide peptide \\
\hline v1.21565 & K08375 & Neuropeptide FF receptor 2 \\
\hline v1.06392 [+ 11 other sequence copies] & K04209 & Neuropeptide $Y$ receptor, invertebrate \\
\hline v1.08609 [+ 31 other sequence copies] & K06819 & Neuropilin 2 \\
\hline v1.11492 [+ 5 other sequence copies] & K03308 & Neurotransmitter:Na+ symporter, NSS family \\
\hline v1.16744 [+ 8 other sequence copies] & K06774 & Neurotrimin \\
\hline v1.05353 & K03176 & Neurotrophic tyrosine kinase receptor type 1 \\
\hline v1.20055 & K04360 & Neurotrophic tyrosine kinase receptor type 2 \\
\hline v1.03803 & K04356 & Neurotrophin 3 \\
\hline v1.09523 & K04803 & Nicotinic acetylcholine receptor alpha-1 (muscle) \\
\hline v1.11940 & K04806 & Nicotinic acetylcholine receptor alpha-4 \\
\hline v1.01548 & K04808 & Nicotinic acetylcholine receptor alpha- 6 \\
\hline v1.05056; v1.12097 & K04809 & Nicotinic acetylcholine receptor alpha-7 \\
\hline v1.07222; v1.11069 & K04810 & Nicotinic acetylcholine receptor alpha-9 \\
\hline v1.18231 [+ 32 other sequence copies] & K05312 & Nicotinic acetylcholine receptor, invertebrate \\
\hline
\end{tabular}


Table $\mathbf{5}$ Neuronal and sensory proteins in the predicted proteome of A. digitifera (Continued)

\begin{tabular}{|c|c|c|}
\hline v1.24404 & K04813 & Nicotinic acetylcholine receptor beta-2 (neuronal) \\
\hline v1.06514; v1.23640 & K04815 & Nicotinic acetylcholine receptor beta-4 \\
\hline v1.18634 & K04816 & Nicotinic acetylcholine receptor delta \\
\hline v1.18231 [+ 32 other sequence copies] & K05312 & Nicotinic acetylcholine receptor, invertebrate \\
\hline v1.05293 [+ 78 other sequence copies] & K02599 & Notch protein \\
\hline v1.15348 [+ 4 other sequence copies] & K04256 & c-Opsin protein \\
\hline v1.01972 & K08385 & G0-Opsin protein \\
\hline v1.13345 [+ 5 other sequence copies] & K04255 & r-Opsin protein \\
\hline v1.00749; v1.03435 & K00504 & Peptidylglycine monooxygenase \\
\hline v1.12323 [+ 11 other sequence copies] & K00678 & Phosphatidylcholine-retinol O-acyltransferase \\
\hline v1.18340 [+ 6 other sequence copies] & K09624 & Protease, serine, 12 (neurotrypsin, motopsin) \\
\hline v1.08030 [+ 9 other sequence copies] & K01931 & Protein neuralized \\
\hline v1.04431 & K09333 & Retina and anterior neural fold homeobox-like protein \\
\hline v1.01789; v1.06542 & K00061 & Retinol dehydrogenase \\
\hline v1.05804 [+ 6 other sequence copies] & K11150 & Retinol dehydrogenase 8 \\
\hline v1.22340; v1.14029 & K11151 & Retinol dehydrogenase 10 \\
\hline v1.24399; v1.07017 & K11154 & Retinol dehydrogenase 16 \\
\hline v1.19667; v1.16885; v1.24371 & K00909 & Rhodopsin kinase \\
\hline v1.12432; v1.15302; v1.07505 & K09516 & all-trans-Retinol 13,14-reductase \\
\hline v1.09104 [+ 6 other sequence copies] & K05613 & Solute carrier family 1 (glial high affinity glutamate transporter), member 2 \\
\hline v1.19779; v1.08769; v1.22032 & K05617 & Solute carrier family 1 (high affinity Asp/glutamate transporter), member 6 \\
\hline v1.19293; v1.19292 & K14387 & Solute carrier family 5 (high affinity choline transporter), member 7 \\
\hline v1.10901; v1.19493 & K05336 & Solute carrier family 6 (neurotransmitter transporter), invertebrate \\
\hline v1.24615 [+ 10 other sequence copies] & K05034 & Solute carrier family 6 (neurotransmitter transporter, GABA) member 1 \\
\hline v1.07932 & K05046 & Solute carrier family 6 (neurotransmitter transporter, GABA) member 13 \\
\hline v1.01817 & K05036 & Solute carrier family 6 (neurotransmitter transporter, dopamine) member 3 \\
\hline v1.20691; v1.16333; v1.15484; v1.02123 & K05038 & Solute carrier family 6 (neurotransmitter transporter, glycine) member 5 \\
\hline v1.15484; v1.15484 & K05042 & Solute carrier family 6 (neurotransmitter transporter, glycine) member 9 \\
\hline v1.18461; v1.09068; v1.02237; v1.20880 & K05333 & Solute carrier family 6 (neurotransmitter transporter) member 18 \\
\hline v1.02239; v1.13836; v1.09067 & K05334 & Solute carrier family 6 (neurotransmitter transporter) member 19 \\
\hline v1.21997 [+ 5 other sequence copies] & K12839 & Survival of motor neuron-related-splicing factor member 30 \\
\hline v1.21997 [+ 6 other sequence copies] & K13129 & Survival motor neuron protein \\
\hline
\end{tabular}

increasing the saturation state of $\mathrm{CaCO}_{3}$ to sustain calcium precipitation [146]. Importantly, located also at the calicoblastic membrane is carbonic anhydrase [149] which is required to catalyse the intermediate step of calcification by the reversible hydration of carbon dioxide $\left(\mathrm{CO}_{2}+\mathrm{H}_{2} \mathrm{O} \Rightarrow \mathrm{HCO}_{3}^{-}+\mathrm{H}^{+}\right)$. In coral phototrophic symbiosis, despite numerous studies describing the well-known phenomenon of light-enhanced calcification, the relationship linking symbiont photosynthesis to coral calcification has been elusive $[150,151]$. Nonetheless, efforts to better understand the calcifying response of scleractinian corals to environmental change and ocean acidification are gaining traction $[149,152,153]$.
Voltage-gated calcium channels (VGCCs) have been examined extensively in mammalian physiology for converting membrane potential into intracellular $\mathrm{Ca}^{2+}$ transients for signalling transduction pathways (reviewed in [154]). VGCC signalling affects cellular processes to include muscle contraction, neuronal excitation, gene transcription, fertilisation, cell differentiation and development, proliferation, hormone release, activation of calcium-dependent protein kinases, cell death via necrosis and apoptosis pathways, phagocytosis and endo/ exocytosis. Remarkably, annotation of the genome of $A$. digitifera reveals sequences encoding homologues of all the VGCC $(\alpha, \alpha \delta, \beta$, and $\gamma)$ subunits of the molecular 
(L, N, P/Q and R) phenotypes expressed in mammalian physiology (Table 6). There are multiple sequences encoding three variants of $\mathrm{Ca}^{2+}$-transporting ATPase, of which at least one is necessary for coral calcification. There is only one sequence match for expressing carbonic anhydrase in the genome of $A$. digitifera, which may reflect the high catalytic efficiency of this calcifying enzyme [155], although a BLAST search of ZoophyteBase does reveal scaffolds with low e-values which on future experimental inspection might uncover multiple copies of this enzyme essential for calcification. There are multiple sequences that express solute carrier $\mathrm{Na}^{+} / \mathrm{Ca}^{2+}$ - and $\mathrm{Na}^{+} /$ $\mathrm{K}^{+} / \mathrm{Ca}^{2+}$-exchange families of transport proteins that with expression of the coral $\mathrm{Ca}^{2+} / \mathrm{H}^{+}$-antiporter may regulate cellular $\mathrm{pH}$ and $\mathrm{Ca}^{2+}$ homeostasis.

Implicit to coral calcification is $\mathrm{Ca}^{2+}$ regulation that affects signalling of other vital cellular functions. Cellular $\mathrm{Ca}^{2+}$ is mediated by the calcium-sensing receptor calmodulin (18 sequence matches) and other messenger calcium-binding effectors (Table 6), including the calcium-binding protein CML (40 protein domain sequence matches). Calcium/calmodulin-protein kinase proteins are arguably key to $\mathrm{Ca}^{2+}$-signalling in coral symbiosis but, with the exception of activation of sperm flagellar motility [156], their precise role has not been elaborated.

\section{Plant-derived proteins}

Endosymbiosis has contributed greatly to eukaryotic evolution, most notably to the genesis of plastids and mitochondria derived from prokaryotic antecedents. Genetic integration by endosymbiont-to-host transfer (EGT) or replacement (EGR) has been a significant force in early metazoan innovation, whereby nuclear transferred genes may even adopt novel functions in the host cell or replace existing versions of the protein that they encode [157]. Prokaryote-to-eukaryotic gene transfer has been widespread in evolution, but examples of genetic exchange between unrelated eukaryotes, such as between algal symbionts and their multicellular eukaryote host, are considered rare (reviewed by $[158,159])$. One such example is $\operatorname{aroB}$ (3-dehydroquinate synthase) transferred to the genome of the sea anemone $N$. vectensis, which sequence best fits that of the dinoflagellate Oxyrrhis marina [119]. Close inspection of the amino acid sequence of the $\operatorname{aro} B$ gene product, as reported by Shinzato et al. [45], clearly shows this protein to be 2-epi-5-epi-valiolone synthase (EVS), a sugar phosphate cyclase orthologue that catalyses the conversion of sedoheptulose 7-phosphate to 2epi-5-epi-valiolone found to be a precursor of the mycosporine-like amino acid (MAA) sunscreen shinorine in the cyanobacterium Anabaena variabilis [160]. Additionally, the EVS gene of $N$. vectensis has a distinctive $O$ methytransferase fusion that is identical in O. marina [161]. The shikimate pathway is essential to apicomplexan parasites of the genera Plasmodium, Toxoplasma and Cryptosporidium and of Tetrahymena ciliates to express a pentafunctional aroM gene similar to that of Ascomycetes, which is thought to have been conveyed by fungal gene transfer to a common ancestral progenitor [162]. In a separate example, $H$. viridis expresses a plant-like ascorbate peroxidase gene (HvAPX1) during oogenesis in both symbiotic and aposymbiotic individuals [163], whereby peroxidase activity is coincident with oogenesis and embryo genesis that in Hydra acts as a ROS scavenger to protect the oocyte from apoptotic degradation [164]. The sacoglossan (sea slug) molluscs Elysia chlorotica and E. viridis (Plakobranchidae) acquire plastids on ingestion of the siphonaceous alga Voucherea litorea (termed "kleptoplasty") and, by maintaining sequestered plastids in an active photosynthetic state, has emerged as a model organism for the transfer of nuclear-encoded plant genes from algal symbiont to its animal host [165]. In this symbiosis, the family of light-harvesting genes $p s b O$, prk (phosphoribokinase) and chlorophyll synthase $(\mathrm{chlG})$ are entrained in the genome of Elysia chlorotica (reviewed in [166,167]), although there is debate whether these genes are transcriptionally expressed (compare [168] and [169]). Also, phylogenomic analysis of the predicted proteins of the aposymbiotic unicellular choanoflagellate Monosiga brevicollis, considered to be a stem progenitor of the animal kingdom [170,171], reveals 103 genes having strong algal affiliations arising from multiple phototrophic donors [172]. Such notable examples illustrate the transfer of algal genes to animal recipients.

KEGG orthology-based annotation of the predicted proteome of $A$. digitifera reveals a plethora of sequences presumed to be of algal origin (Table 7). Like $E$. chlorotica, the coral genome has encoded the photosystem II (PSII) protein PsbO of the oxygen-evolving complex of photosynthesis, as well as the PSII lightharvesting complex protein PsbL that is important in protecting PSII from photo-inactivation [173]. Encoded also are the photosystem I subunit proteins PsaI and $\mathrm{PsaO}$. Additionally encoded are the photosystem P840 reaction center cytochrome c551 ( $\mathrm{PscC}$ ) protein and the photosynthetic reaction center $M$ subunit protein, the light-harvesting proteins complex 1 alpha (PufA), the complex II chlorophyll $a / b$ binding protein 6 (LHCB6), the cyanobacterial phycobilisome proteins AcpF and AcpG, the phycocyanin-associated antenna protein $\mathrm{CpcD}$, the phycocyanobilin lyase protein $\mathrm{CpcF}$ and the phycoerythrinassociated linker protein CpeS. Like E. chlorotica, the coral genome encodes chlorophyll synthase (ChlG), a chlorophyll transporter protein PucC, a light-independent nitrogenaselike protochlorophyllide reductase enzyme that is sensitive to oxygen [174] and a red chlorophyll reductase essential to the detoxification of photodynamic chlorophyll catabolites arising from plant/algal senescence [175]. Three 
Table 6 Calcification and $\mathrm{Ca}^{2+}$-signalling proteins in the predicted proteome of $A$. digitifera

\begin{tabular}{|c|c|c|}
\hline Gene sequence & KEGG Orthology & Encoded protein description \\
\hline v1.06452; v1.06451; v1.24424; v1.16923 & K07300 & $\mathrm{Ca} 2+\mathrm{H}+$ antiporter \\
\hline v1.01669 [+ 9 other sequence copies] & K01537 & Ca2+-transporting ATPase \\
\hline v1.22367; v1.22366; v1.22365 & K05850 & $\mathrm{Ca} 2+$ transporting ATPase, plasma membrane \\
\hline v1.19074 & K05853 & $\mathrm{Ca} 2+$ transporting ATPase, sarcoplasmic/endoplasmic reticulum \\
\hline v1.22416; v1.22417; v1.15682; v1.00750 & K14757 & Calbindin D28 \\
\hline v1.24568 [+ 9 other sequence copies] & K01672 & Carbonic anhydrase \\
\hline v1.09241 & K08272 & Calcium binding protein 39 \\
\hline v1.02323 [+ 39 other sequence copies] & K13448 & Calcium-binding protein CML \\
\hline v1.05162 [+ 21 other sequence copies] & K13412 & Calcium-dependent protein kinase \\
\hline v1.09352 & K07359 & Calcium/calmodulin-dependent protein kinase kinase \\
\hline v1.06475; v1.07555;v1.00945; v1.00159; v1.21122 & K08794 & Calcium/calmodulin-dependent protein kinase I \\
\hline v1.06475; v1.01061; v1.21150; v1.22443 & K04515 & Calcium/calmodulin-dependent protein kinase II \\
\hline v1.00159 & K05869 & Calcium/calmodulin-dependent protein kinase IV \\
\hline v1.21927; v1.01218; v1.22226; v1.06623; v1.13703 & K06103 & Calcium/calmodulin-dependent serine protein kinase \\
\hline v1.13460 & K08284 & Calcium channel MID1 \\
\hline v1.20738; v1.01401 & K12841 & Calcium homeostasis endoplasmic reticulum protein \\
\hline v1.22794 [+ 11 other sequence copies] & K04612 & Calcium-sensing receptor \\
\hline v1.10079 [+ 17 other sequence copies] & K02183 & Calmodulin \\
\hline v1.10994 & K14734 & S100 calcium binding protein $\mathrm{G}$ \\
\hline v1.02488 [+ 14 other sequence copies] & K05849 & Solute carrier family 8 (sodium/calcium exchanger) \\
\hline v1.23153 [+ 9 other sequence copies] & K13749 & Solute carrier family 24 (sodium/potassium/calcium exchanger) \\
\hline v1.14863 & K12304 & Soluble calcium-activated nucleotidase 1 \\
\hline v1.18656 [+ 13 other sequence copies] & K04858 & Voltage-dependent calcium channel alpha-2/delta-1 \\
\hline v1.13222 & K04860 & Voltage-dependent calcium channel alpha-2/delta-3 \\
\hline v1.08078 [+ 9 other sequence copies] & K05315 & Voltage-dependent calcium channel alpha 1, invertebrate \\
\hline v1.03896 [+ 6 other sequence copies] & K05316 & Voltage-dependent calcium channel alpha-2/delta, invertebrate \\
\hline v1.04798 & K05317 & Voltage-dependent calcium channel beta, invertebrate \\
\hline v1.22788 & K04863 & Voltage-dependent calcium channel beta-2 \\
\hline v1.09999 & K04872 & Voltage-dependent calcium channel gamma-7 \\
\hline v1.02505 & K04873 & Voltage-dependent calcium channel gamma-8 \\
\hline v1.03648[+ 6 other sequence copies] & K04850 & Voltage-dependent calcium channel L type alpha- $1 C$ \\
\hline v1.03648; v1.17267 & K04851 & Voltage-dependent calcium channel L type alpha-1D \\
\hline v1.03648; v1.13219; v1.21895 & K04857 & Voltage-dependent calcium channel L type alpha-1S \\
\hline v1.06313; v1.01656; v1.23096 & K04344 & Voltage-dependent calcium channel P/Q type alpha-1A \\
\hline v1.08078 [+ 10 other sequence copies] & K04849 & Voltage-dependent calcium channel N type alpha-1B \\
\hline v1.07968 & K04852 & Voltage-dependent calcium channel R type alpha-1E \\
\hline v1.01364; v1.13467; v1.08705 & K04854 & Voltage-dependent calcium channel T type alpha-1G \\
\hline v1.15414; v1.14241; v1.09595 & K04855 & Voltage-dependent calcium channel T type alpha-1H \\
\hline
\end{tabular}

chlorosome proteins of the photosynthetic antenna complex of green sulphur bacteria, a bacteriochlorophyll methyltransferase involved in BChl $c$ biosynthesis [176] and the retinylidene bacteriorhodopsin of phototrophic Archaea are also encoded in the coral genome. Present are genes encoding subunit 6 of the cytochrome $B_{6} f$ complex that links PSII and PSI via the plastoquinone pool, together with chloroplast ferredoxin-like NapH and NapG proteins and their $2 \mathrm{Fe}-2 \mathrm{~S}$ cluster protein. The coral genome, however, encodes sequences for $\mathrm{NAD}^{+}$-ferredoxin 
Table 7 Plant-derived proteins in the predicted proteome of $A$. digitifera

\begin{tabular}{|c|c|c|}
\hline Gene sequence & KEGG Orthology & Encoded protein description \\
\hline v1.14452 & K09843 & (+)-Abscisic acid 8'-hydroxylase \\
\hline v1.18868 & K14496 & Abscisic acid receptor PYR/PYL family (PYL) \\
\hline v1.21983; v1.05890 & K03342 & p-Aminobenzoate synthetase / 4-amino-4-deoxychorismate lyase (PabBC) \\
\hline v1.15436 & K02822 & Ascorbate-specific IIB component, PTS system (PTS-Ula-EiiB) \\
\hline v1.11187; v1.13966 & K00423 & L-Ascorbate oxidase \\
\hline v1.20081; v1.22465 & K13604 & Bacteriochlorophyll C20 methyltransferase (BchU) \\
\hline v1.07723 & K04641 & Bacteriorhodopsin (BoP) \\
\hline v1.21858 & K04040 & Chlorophyll synthase (ChIG) \\
\hline v1.01742 & K08945 & Chlorosome envelope protein A (CsmA) \\
\hline v1.04797; v1.14208 & K08946 & Chlorosome envelope protein B (CsmB) \\
\hline v1.18698 & K08948 & Chlorosome envelope protein D (CamD) \\
\hline v1.18637 & K02642 & Cytochrome $b_{6} f$ complex subunit 6 (PetL) \\
\hline v1.21101; v1.14192; v1.14548 & K01735 & 3-Dehydroquinate synthase (AroB) \\
\hline v1.05796 & K10210 & 4,4'-Diaponeurosporene oxidase (carotenoid biosynthesis; CrtP) \\
\hline v1.11730 & K04755 & Ferredoxin, 2Fe-2S (FdX) \\
\hline v1.19154; v1.00014 & K00532 & Ferredoxin hydrogenase \\
\hline v1.00014 & K00534 & Ferredoxin hydrogenase small subunit \\
\hline v1.17698; v1.06031; v1.16647 & K02574 & Ferredoxin-type protein $(\mathrm{NapH})$ \\
\hline v1.23058 & K02573 & Ferredoxin-type protein (NapG) \\
\hline v1.08414 & K08926 & Light-harvesting complex 1 alpha chain (PufA) \\
\hline v1.21458 & K08917 & Light-harvesting complex II chlorophyll a/b binding protein 6 (LHCB6) \\
\hline v1.03743 & K08226 & MFS transporter, BCD family, chlorophyll transporter (PucC) \\
\hline v1.13030; v1.08678 & K13413 & Mitogen-activated protein kinase kinase 4/5, plant ((MKK4_5P) \\
\hline v1.02429; v1.10744; v1.03340 & K08929 & Photosynthetic reaction center M subunit (PufM) \\
\hline v1.03631 & K02696 & Photosystem I subunit VIII (Psal) \\
\hline v1.11432 & K14332 & Photosystem I subunit (PsaO) \\
\hline v1.17422 & K02713 & Photosystem II protein (PsbL) \\
\hline v1.18303 & K02716 & Photosystem II oxygen-evolving enhancer protein 1 (PsbO) \\
\hline v1.12300; v1.21136 & K08942 & Photosystem P840 reaction center cytochrome c551 ((PscC) \\
\hline v1.00280 & K02097 & Phycobilisome core component 9 (AcpF) \\
\hline v1.10967 & K02290 & Phycobilisome rod-core linker protein (AcpG) \\
\hline v1.02166 & K02287 & Phycocyanin-associated, rod protein (CpcD) \\
\hline v1.19642; v1.07305; v1.19572; v1.01248 & K02289 & Phycocyanobilin lyase beta subunit (CpcF) \\
\hline v1.10441 & K05382 & Phycoerythrin-associated linker protein (CpeS) \\
\hline v1.13406 & K10027 & Phytoene dehydrogenase (desaturase; $\mathrm{Crtl}$ ) \\
\hline v1.18809; v1.06199 & K02291 & Phytoene synthase (CrtB) \\
\hline v1.20411; v1.02037; v1.14064; v1.21095 & K09060 & Plant G-box-binding factor (GBF) \\
\hline v1.10035 & K00218 & Protochlorophyllide reductase [NifEN-like; Por] \\
\hline v1.21846 & K05358 & Quinate dehydrogenase (QuiA) \\
\hline v1.03127 & K13545 & Red chlorophyll catabolite reductase (ACD2) \\
\hline v1.05899 & K00891 & Shikimate kinase (AroK, AroL) \\
\hline v1.21101; v1.14192; v1.05899 & K13829 & Shikimate kinase / 3-dehydroquinate synthase (AroKB) \\
\hline v1.12938 & K08500 & Syntaxin of plants (SYP6) \\
\hline v1.06575 & K08506 & Syntaxin of plants (SYP7) \\
\hline
\end{tabular}


Table 7 Plant-derived proteins in the predicted proteome of A. digitifera (Continued)

\begin{tabular}{lll}
\hline v1.04929 & K09834 & Tocopherol cyclase (VTE1, SXD1) \\
v1.01022 & K05928 & Tocopherol O-methyltransferase \\
v1.05457 & K09838 & Zeaxanthin epoxidase (ZEP, ABA1) \\
\hline
\end{tabular}

reductase (HcaD; not tablulated), rather than the required $\mathrm{NADP}^{+}$-ferredoxin reductase of photosynthesis. Annotation of the $A$. digitifera genome revealed genes unexpectedly encoding ferredoxin hydrogenase [EC:1.12.7.2] and that of its small subunit protein (Table 7) involved in light-dependent production of molecular hydrogen having its [Fe-Fe]-cluster coupled to the photosynthetic transport chain via a chargetransfer complex with ferredoxin (see [177]).

Like $N$. vectensis and the dinoflagellate Oxyrrhis marina, the genome of $A$. digitifera encodes an $O$-methyltransferase which is immediately downstream of EVS, but the two genes are not fused. Using a ZoophyteBase BlastP search, the $O$-methyltransferase showed little sequence homology with the corresponding protein of $A$. variabilis (e-value of $6.972 \mathrm{E}^{-2}$ and Bit score of 34.27), whereas the EVS protein shared $87 \%$ absolute sequence identity to the A. variabilis EVS protein. What role, if any, these two genes play in mycosporine-like amino acid (MAA) biosynthesis in $A$. digitifera has yet to be determined, although it has been suggested from the transcriptome of Acropora microphthalma that MAA biosynthesis proceeds from a branch point at 3-dehydroquinate of the shikimic acid pathway as a shared metabolic adaptation between the coral host and its symbiotic zooxanthellae [40]. The 3-dehydroquinate synthase enzyme of the shikimic acid pathway, thought to be a key intermediate in an alternative MAA biosynthetic pathway in A. variabilis [178], is instead encoded by the fused aroKB gene of $A$. digitifera (Table 7 ). Additional shikimate proteins of the predicted proteome, although not limited to phototrophs, are shikimate kinase (AroK), quinate dehydrogenase (QuiA) and the conjoined $p$-aminobenzoate synthase and 4-amino-4-deoxychlorismate lysate (PabBC) enzyme necessary for folate biosynthesis [179]. Other plant-related gene homologues include the phytohormone abscisic acid receptor protein (PabBC) and its cytochrome P450 monooxygenase abscisic acid 8'-hydroxylase, L-ascorbate oxidase and PTS system degrading enzymes, the unique SYP6 and SYP7 syntaxins of plant vesicular transport, tocopherol cyclase and a tocopherol $O$ methyltransferase enzyme that converts $\gamma$-tocopherol to $\alpha$-tocopherol. Essential for carotene biosynthesis are phytoene synthase (CrtB) and phytoene dehydrogenase (CrtI) enzymes. Significantly, encoded within the coral genome is zeaxanthin epoxidase that is essential for abscisic acid biosynthesis and is a key enzyme in the xanthophyll cycle of plants and algae to impart oxidative stress tolerance.
Given that viruses often mediate gene transfer processes, it is intriguing that certain bacteriophages of marine Synechococcus and Prochlorococcus cyanobacteria are reported to carry genes encoding the photosynthesis D1 $(p s b A)$, and D2 (psbD) proteins, a high-light inducible protein (HLIP) $[180,181]$ and the photosynthetic electron transport plastocyanin (petE) and ferredoxin (petF) proteins thought to enhance the photosynthetic fitness of their host [182-184]. Accordingly, it has been suggested that the transfer of $p s b A$ by viruses associated with Symbiodinium could lessen the severity of thermal impairment to PSII and the response of corals to thermal bleaching [185]. It is yet unknown if phages or dinoflagellate-infecting viruses [186], particularly those of Symbiodinium [187], may affect gene transfer leading to complementary (or "shared") metabolic adaptations of symbiosis $[119,188]$.

\section{Proteins of nitrogen metabolism}

It is well accepted that intracellular Symbiodinium spp. provide reduced carbon for coral heterotrophic metabolism by photosynthetic carbon fixation. Because of this metabolic relationship, light is a critical feature in the bioenergetics of coral symbiosis [189]. The algal photosynthate translocated to corals, however, is deficient in nitrogen at levels necessary to sustain autotrophic growth. While corals can assimilate fixed nitrogen from surrounding seawater [190], "recycled" nitrogen within the symbiosis may account for as much as $90 \%$ of the photosynthetic nitrogen demand [191]. It would not be surprising then that light would have a strong influence on the uptake and retention of ammonium by symbiotic corals. Consequently, corals excrete excess ammonium in darkness [192], and in light excretion is induced by treatment with the photosynthetic electron transport inhibitor 3-(3,4-diclorophenyl)-1,1-dimethylurea (DCMU) [193]. Since ammonia is the product of nitrogen fixation, these observations suggest that the coral holobiont may fix nitrogen in the dark, or when photosynthesis is repressed, during which coral tissues are hypoxic [194], and nitrogenase activity is not inactivated by molecular oxygen [195].

Tropical coral reefs are typically surrounded by lownutrient oceanic waters of low productivity but, paradoxically, the waters of coral reefs often have elevated levels of inorganic nitrogen $[196,197]$ attributed to high rates of nitrogen fixation. While nitrogen fixation from diazotrophic 
epiphytes of the coral reef substrata and sediments $[197,198]$ and diazotrophic bacterioplankton of the coral reef lagoon [199] provide substantial quantities of fixed nitrogen for assimilation by the coral reef, mass-balance estimates show this input to be less than the community's annual nitrogen demand [200]. Endolithic nitrogen-fixing bacteria are abundant in the skeleton of living corals where they benefit from organic carbon excreted by overlaying coral tissues to provide a ready source of energy for dinitrogen reduction [201]. Additionally, intracellular nitrogen-fixing cyanobacteria are reported to coexist with dinoflagellate symbionts in the tissues of Monastraea cavernosa and to functionally express nitrogenase activity [202]. Corals also harbour a diverse assemblage of heterotrophic microorganisms in their skeleton, tissues and lipid-rich mucus (reviewed in [203]), and these communities include large populations of diazotrophic bacteria [204,205], and archaea [206]. Apart from nitrogen fixation, the coral microbiota contributes to other nitrogen-cycling processes, such as nitrification, ammonification and denitrification $[207,208]$. We were surprised to find several nitrogen fixation and cycling proteins encoded in the genome of $A$. digitifera (Table 8 ), notably a nitrogen fixation NifU-like protein, the Nif-specific regulatory protein (NifA), the regulatory $\mathrm{NAD}(+)$-dinitrogen-reductase ADP-Dribosylastransferase protein, a nitrifying ammonia monooxygenase enzyme and nitrate reductase, which are usually expressed only by prokaryotic microorganisms.

The presence of genes encoding proteins involved in nitrogen fixation raises speculation that corals may contribute directly to, or perhaps co-regulate, certain processes that catalyse the reduction of dinitrogen $\left(\mathrm{N}_{2}\right)$ to ammonia $\left(\mathrm{NH}_{3}\right)$ by the enzyme nitrogenase reductase $(\mathrm{NifH})$. The functional NifH enzyme is a binary protein composed of a molybdenum-iron $(\mathrm{MoFe})$ protein $(\mathrm{NifB} /$ NifDK), or its NifEN homologue, fused with a FeMocofactor (FeMoco) protein [209]. While genes encoding NifB, NifDK (or NifEN) and their FeMo-cofactor do not appear in the genome of $A$. digitifera, a gene encoding the NifEN-like protein protochlorophyllide oxidoreductase (POR) is present (Table 8). POR has all three subunits with high similarity to the assembled MoFe nitrogenase [210], but this homologue is unlikely to be effective in nitrogen reduction [211,212] since its activity is light dependent [213] when tissues are highly oxic [193]. The NifU protein encoded in the coral genome preassembles the metallocatalytic Fe-S clusters for maturation of nitrogenase [214], but its assemblage without NifS, a cysteine desulfurase needed for [Fe-S] cluster assembly [215], would be incomplete, and its pre-nitrogenase receptor is also missing. Yet, the coral does have the niff gene that encodes pyruvate:flavodoxin oxidoreductase required for electron transport in nitrogenase reduction [216]. The regulatory NifA protein encoded in the coral genome might activate, on stimulation by the integration host factor (INF), transcription of nitrogen fixation (nif) operons of RNA polymerase [217], and both of these proteins are encoded in the coral genome. Additional to this transcriptional control, post-translational nitrogenase activity is controlled by reversible ADP-ribosylation of a specific arginine residue in the nitrogenase complex [218]. NAD (+)-dinitrogen-reductase ADP-D-ribosyltransferase (DraT) inactivates the nitrogenase complex while ADP-ribosylglycohydrolase (DraG) removes the ADP-ribose moiety to restore nitrogenase activity, and both of these enzymes are encoded in the coral genome. Given that genes encoding essential constituent proteins of nitrogenase assembly appear incomplete, corals are unlikely to fix nitrogen per se, but co-opted elements of the coral genome to regulate processes of nitrogen fixation by its diazotrophic consortia is a prospect worthy of exploration [219].

Nitrofying/nitrifying bacteria and archaea express the enzyme ammonia monooxygenase that converts fixed ammonia to nitrite (via hydroxylamine) and the enzyme nitrite (oxido)reductase completes the oxidation of nitrite to nitrate, and both of these enzymes are entrained in the genome of $A$. digitifera (Table 8). The ammonia monooxygenase subunit $\mathrm{A}(\mathrm{amo} A)$ of archaeal consorts has been described in nine species of coral from four reef locations [220], but the presence of $a m o A$ in the coral genome, together with encoded ammonium transport proteins, was not anticipated. Another protein of prokaryotic origin encoded in the coral genome is nitrate reductase (periplasmic, assimilatory and respiratory), the latter being required for anaerobic respiration by bacteria [221], and unlike the nitrate reductase family of sulphite oxidase enzymes in eukaryotes, the nitrate reductases of prokaryotes (K00363) belong to the DMSO reductase family of enzymes. Also encoded in the coral genome are a nitrite transporter ( $\mathrm{NirC}$ ) and a formatedependent nitrite reductase (NrfA) required for nitrite ammonification [222]. In addition to nitrite reduction, NrfA reduces nitric oxide, hydroxylamine, nitrous oxide and sulphite, the last providing a metabolic link between nitrogen and sulphur cycling in coral metabolism. Other enzymes of nitrogen metabolism encoded in the coral genome are the carbamoyl-phosphate synthase family of enzymes [223] that catalyses the ATP-dependent synthesis of carbamoyl phosphate used for the production of urea (ornithine cycle) to provide a ready store of fixed-N in the urea-nitrogen metabolism of corals [224]. Another nitrogen source comes from glutamate dehydrogenase $(\mathrm{GDH})$ that reversibly converts glutamate to $\alpha$-ketoglutarate with liberation of ammonia, and as expected [225], this enzyme is encoded in the coral genome, together with the prokaryotic nitrogen regulatory protein PII of glutamine synthase, which in bacteria is activated in response to nitrogen availability. Encoded also is 
Table 8 Proteins of nitrogen metabolism in the predicted proteome of $A$. digitifera

\begin{tabular}{|c|c|c|}
\hline Gene sequence & KEGG Orthology & Encoded protein description \\
\hline v1.23444; v1.09133; v1.23443 & K05521 & ADP-ribosylglycohydrolase (DraG) \\
\hline v1.09202 & K10944 & Ammonia monooxygenase subunit $A$ \\
\hline v1.03645 [+ 8 other sequence copies] & K03320 & Ammonium transporter, Amt family \\
\hline v1.12268; v1.12269 & K06580 & Ammonium transporter Rh \\
\hline v1.02406 & K01954 & Carbamoyl-phosphate synthase (CPS) \\
\hline v1.01524; v1.18283; v1.18284 & K01948 & Carbamoyl-phosphate synthase (CPS, ammonia) \\
\hline v1.01615 & K04016 & Formate-dependent nitrite reductase (NrfA) \\
\hline v1.16277; v1.23483; v1.13667; v1.22675 & K00261 & Glutamate dehydrogenase $(\mathrm{NAD}(\mathrm{P})+)$ \\
\hline v1.17166; v1.11089 & K01745 & Histidine ammonia-lyase \\
\hline v1.22825; v1.08034;v1.08520 & K05123 & Integration host cell factor (INF) subunit beta \\
\hline v1.11343 & K05951 & NAD+-dinitrogen-reductase ADP-D-ribosyltransferase (DraT) \\
\hline v1.00547 & K02584 & Nif-specific regulatory protein (NifA) \\
\hline v1.18869 & K00371 & Nitrate reductase 1 , beta subunit \\
\hline v1.06763 & K08346 & Nitrate reductase 2 , beta subunit \\
\hline v1.14858; v1.00685; v1.23148 & K05916 & Nitric oxide dioxygenase \\
\hline v1.16954 [+ 5 other sequence copies] & K02448 & Nitric oxide reductase NorD protein \\
\hline v1.06115 & K02164 & Nitric oxide reductase NorE protein \\
\hline v1.17629 [+ 12 other sequence copies] & K04748 & Nitric oxide reductase NorQ protein \\
\hline v1.24077 [+ 4 other sequence copies] & K13125 & Nitric oxide synthase-interacting protein \\
\hline v1.21801; v1.05719; v1.23577; v1.19464 & K13253 & Nitric-oxide synthase, invertebrate \\
\hline v1.05980 & K00363 & Nitrite reductase $(\mathrm{NAD}(\mathrm{P}) \mathrm{H})$ small subunit \\
\hline v1.00101 & K02598 & Nitrite transporter NirC \\
\hline v1.02355; v1.18772 & K04488 & Nitrogen fixation protein NifU \\
\hline v1.17812 & K02589 & Nitrogen regulatory protein PII 1 \\
\hline v1.09150 & K02570 & Periplasmic nitrate reductase NapD \\
\hline v1.01560 & K02571 & Periplasmic nitrate reductase NapE \\
\hline v1.10035 & K00218 & Protochlorophyllide reductase [NifEN-like] \\
\hline v1.08939 & K03737 & Pyruvate-flavodoxin reductase (NifJ) \\
\hline v1.17373 & K00365 & Urate oxidase \\
\hline v1.13217 & K01427 & Urease \\
\hline v1.16409 [+ 5 other sequence copies] & K03187 & Urease accessory protein \\
\hline v1.13217 & K01429 & Urease subunit beta \\
\hline v1.13217 & K14048 & Urease subunit gamma/beta \\
\hline v1.12211 [+ 4 other sequence copies] & K00106 & Xanthine dehydrogenase/oxidase \\
\hline v1.12212 & K13481 & Xanthine dehydrogenase small subunit \\
\hline
\end{tabular}

(Excluding amino acid and pyrimidine/purine nucleotide synthesis or metabolism).

histidine ammonia-lyase (histidase) that liberates ammonia (and urocanic acid) from cytosolic stores of histidine. It is now accepted that uric acid deposits accumulated by symbiotic algae provide a significant store of nitrogen for the coral holobiont [226], so it is noteworthy that the coral genome encodes urate oxidase (uricase) to catalyse uric acid oxidation to allanotoin from which urea and ureidoglycolate are produced in a reaction catalysed by allantoicase (allantoate amidinohydrolase), both of which known isoforms are present in the coral genome. Encoded in the coral genome is also urease to catalyse the hydrolysis of urea, presumably excreted by its algal symbionts, with the release of carbon dioxide and ammonia to meet the nitrogen demand of the coral holobiont during periods of low nitrogen availability. Similarly, xanthine dehydrogenase (xanthine: $\mathrm{NAD}^{+}$-oxidoreductase) acts by oxidation on a variety of purines, 
including hypoxanthine, to yield urate for the recycling of nitrogen in coral nutrition. Many of the aforementioned proteins of nitrogen metabolism, including Nif proteins, have been detected in the proteome of an endosymbiontenriched fraction of the coral S. pistillata [39].

Notwithstanding consideration of the rapid diffusion rate of nitric oxide $(\mathrm{NO})$ or its apparent short biological half-life [227], there is debate about the provenance of endogenously produced $\mathrm{NO}$ in signalling the bleaching of corals in response to environmental stress. Elevated nitric oxide synthase (NOS) activity and NO production in algal symbionts has been attributed to the thermal stress response of corals $[228,229]$, whereas the host is ascribed to be the major source of NO during exposure to elevated temperature $[230,231]$. While our annotation may not resolve this dispute, we show (Table 8) that nitric oxide synthase enzymes (Nor D, Nor E, Nor Q and an invertebrate NOS protein) are encoded in the genome of $A$. digitifera, together with a nitric oxideinteracting protein (NOIP) that in higher animals regulates neuronal NOS activity [232]. Nitric oxide is an intermediate of nitrite reduction catalysed by nitrite reductase (NIR), which by further reduction produces ammonia. The coral genome also encodes nitric oxide dioxygenase (NOD) that converts nitric oxide to nitrate. Accordingly, enhanced expression of NIR (NO reduction) or NOD (NO oxidation) could ameliorate the NOsignalling response of coral bleaching presumed activated by environmental stress.

\section{DNA repair}

Cellular DNA is prone to damage caused by the products of normal metabolism and by exogenous agents. Damage to DNA from metabolic processes include the oxidation of nucleobases and strand interruptions by the production of reactive oxygen species (ROS), from alkylation of nucleotide bases, from the hydrolysis of bases causing deamination, depurination and depyrimidination, and from the mismatch of base pairs from errors in DNA replication. Damage affected by external agents include exposure to UV light causing pyrimidine dimerization and free radicalinduced damage, exposure to ionising radiation causing DNA strand breaks, thermal disruption causing hydrolytic depurination and single-strand breaks, and by xenobiotic contamination to cause DNA adduct formation, nucleobase oxidation and DNA crosslinking. Most of these lesions affect structural changes to DNA that alter or prevent replication and gene transcription at the site of DNA damage. Thus, recognition and repair of DNA abnormalities are vital processes essential to maintain the genetic integrity of the coral genome. Since there are multiple pathways causing DNA damage at diverse molecular sites, there are likewise diverse and overlapping processes available to repair cellular DNA damage. Of the many nuclear repair processes, photoreactivation (photolyase), base excision repair and nucleotide excision repair are the main elements for the repair of cellular DNA damage.

Exposure to sunlight is an absolute requirement for phototrophic symbiosis, but excessive exposure of corals to solar ultraviolet radiation can inflict direct damage to DNA by pyrimidine dimerization and 6-4 photoadduct formation and cause indirect damage by the production of ROS to initiate free-radical damage. While there have been abundant studies on the sensitivity of corals to solar ultraviolet radiation, only a few have examined the effects of solar UV to cause DNA damage. Photoreactivation has been shown to be an important repair pathway for reversing UV-activated DNA damage in adult coral [233] and coral planulae [234]. UV damage to DNA was first demonstrated by the detection of unrepaired cyclobutane pyrimidine dimers (CPDs) in the host tissues and algal symbionts of the coral Porities porites, in which CPDs had increased in a UV dose-dependent manner [235], whereas CPDs and 6-4 pyrimidine-pyrimidone photoadducts in the coral Montipora verrucosa holobiont were correlated inversely with levels of coral "sunscreen" protection [236]. The effects of solar UV radiation causing DNA lesions in coral have been determined by use of the comet assay [237], and UV-induced DNA damage and repair has been examined in the symbiotic anemone Aiptasia pallida [238]. The comet assay showed also that DNA lesions in coral planulae had increased on acquiring algal symbionts, presumably from greater ROS production resulting as a by-product of photosynthesis [239]. Iron-induced oxidative stress was found likewise to enhance DNA damage in the coral Pocillopora damicornis as determined by the occurrence of DNA apurinic/apyrimidinic sites caused by hydrolytic lesions [240]. Significantly, DNA damage in the host and algal symbionts of the coral Montastraea faveolata was found to occur simultaneously during thermal "bleaching" stress, and DNA damage is further enhanced on exposure to greater irradiances of solar radiation [241]. Nevertheless, despite the serious risk of unrepaired DNA damage to coral survival, the DNA repair processes of corals to mitigate the detrimental effects of environmental stress have not been adequately characterised at the transcriptome level of expression [29,242].

Our annotation of the sequenced genome of $A$. digitifera has revealed genes encoding a large repertoire of DNA repairing enzymes and their adaptor proteins (Table 9). Given strong evidence for DNA photoreactivation in corals having been reported [233,234], it was surprising to find only one gene in single copy that encodes a sole photolyase enzyme for reversing pyrimidine dimer and 64 photoadduct formation. Notably, we found genes encoding 6 members of the ERCC family of nucleotide excision repair enzymes, together with the UV excision repair protein RAD23, for the repair of UV-induced DNA damage. 
Table 9 DNA repair proteins in the predicted proteome of $A$. digitifera

\begin{tabular}{|c|c|c|}
\hline Gene sequence & KEGG Orthology & Encoded protein description \\
\hline v1.02961; v1.13402 & K03575 & A/G-specific adenine glycosylase (MutY) \\
\hline v1.11766 & K03919 & Alkylated DNA repair protein \\
\hline v1.04821 & K10765 & Alkylated DNA repair protein alkB homologue 1 \\
\hline v1.02479 & K10766 & Alkylated DNA repair protein alkB homologue 4 \\
\hline v1.20302 & K10767 & Alkylated DNA repair protein alkB homologue 5 \\
\hline v1.24450 & K10768 & Alkylated DNA repair protein alkB homologue 6 \\
\hline v1.02766; v1.09413 & K10770 & Alkylated DNA repair protein alkB homologue 8 \\
\hline v1.01590 [+ 4 other sequence copies] & K10884 & ATP-dependent DNA helicase 2 subunit 1 \\
\hline v1.18810; v1.03166; v1.08449 & K10885 & ATP-dependent DNA helicase 2 subunit 2 \\
\hline v1.08013 & K03722 & ATP-dependent DNA helicase DinG \\
\hline v1.03542 & K14635 & ATP-dependent DNA helicase MPH1 \\
\hline v1.06737 [+ 5 other sequence copies] & K15255 & ATP-dependent DNA helicase PIF1 \\
\hline v1.17360; v1.21235 & K10899 & ATP-dependent DNA helicase Q1 \\
\hline v1.01081 [+ 8 other sequence copies] & K10730 & ATP-dependent DNA helicase Q4 \\
\hline V1.16859 & K10902 & ATP-dependent DNA helicase Q5 \\
\hline v1.11661 [+ 19 other sequence copies] & K03654 & ATP-dependent DNA helicase RecQ \\
\hline v1.20397 & K03656 & ATP-dependent DNA helicase Rep \\
\hline v1.18049; v1.07731; v1.05830 & K10905 & ATR interacting protein \\
\hline v1.01679 & K01669 & Deoxyribodipyrimidine photo-lyase \\
\hline v1.03410; v1.12968; v1.00865; v1.16876 & K10887 & DNA cross-link repair $1 C$ protein \\
\hline v1.07474; v1.07473; v1.01809 & K10610 & DNA damage-binding protein 1 \\
\hline v1.13116; v1.03378; v1.16328 & K10140 & DNA damage-binding protein 2 \\
\hline v1.17099 [+ 5 other sequence copies] & K11885 & DNA damage-inducible protein 1 \\
\hline v1.05469 & K06663 & DNA damage checkpoint protein \\
\hline v1.02859; v1.14719; v1.21030; v1.10920 & K04452 & DNA damage-inducible transcript 3 \\
\hline v1.02191 & K10844 & DNA excision repair protein ERCC-2 \\
\hline v1.19108 [+ 5 other sequence copies] & K10843 & DNA excision repair protein ERCC-3 \\
\hline v1.22267 [+ 4 other sequence copies] & K10848 & DNA excision repair protein ERCC-4 \\
\hline v1.15137 [+ 5 other sequence copies] & K10846 & DNA excision repair protein ERCC-5 \\
\hline v1.18550; v1.02606; v1.14935; v1.08831 & K10841 & DNA excision repair protein ERCC-6 \\
\hline v1.20045; v1.01844; v1.11724; v1.03203 & K10570 & DNA excision repair protein ERCC-8 \\
\hline v1.15430; v1.03058 & K03658 & DNA helicase IV \\
\hline v1.00228 [+ 4 other sequence copies] & K11665 & DNA helicase INO80 \\
\hline v1.00136; v1.0678; v1.21529 & K10776 & DNA ligase 3 \\
\hline v1.23293; v1.19418; v1.23430; v1.15721 & K10777 & DNA ligase 4 \\
\hline v1.19248 & K07458 & DNA mismatch endonuclease, patch repair protein \\
\hline v1.19011 & K08739 & DNA mismatch repair protein MLH3 \\
\hline v1.11513; v1.11449 & K08735 & DNA mismatch repair protein $\mathrm{MSH} 2$ \\
\hline v1.14781 & K08736 & DNA mismatch repair protein MSH3 \\
\hline v1.05696; v1.22444; v1.19162 & K08740 & DNA mismatch repair protein $\mathrm{MSH} 4$ \\
\hline v1.04904 & K08741 & DNA mismatch repair protein MSH5 \\
\hline v1.15360; v1.19426; v1.08585 & K08737 & DNA mismatch repair protein MSH6 \\
\hline v1.02429 [+ 8 other sequence copies] & K03572 & DNA mismatch repair protein MutL \\
\hline v1.03990 & K03555 & DNA mismatch repair protein MutS \\
\hline
\end{tabular}


Table 9 DNA repair proteins in the predicted proteome of A. digitifera (Continued)

\begin{tabular}{|c|c|c|}
\hline v1.14015 & K07456 & DNA mismatch repair protein MutS2 \\
\hline v1.08443 & K10864 & DNA mismatch repair protein PMS1 \\
\hline v1.15229 & K10858 & DNA mismatch repair protein PMS2 \\
\hline v1.08658; v1.14152; v1.01681 & K15082 & DNA repair protein RAD7 \\
\hline v1.16407 [+ 27 other sequence copies] & K10866 & DNA repair protein RAD50 \\
\hline v1.22193 & K04482 & DNA repair protein RAD51 \\
\hline v1.02646; v1.22076 & K10958 & DNA repair protein RAD57 \\
\hline v1.15671 [+ 4 other sequence copies] & K04483 & DNA repair protein RadA \\
\hline v1.16193; v1.19033 & K04485 & DNA repair protein RadA/Sms \\
\hline v1.16079; v1.07685 & K04484 & DNA repair protein $\operatorname{RadB}$ \\
\hline v1.21363; v1.22360; v1.02900 & K03584 & DNA repair protein $\mathrm{RecO}$ (recombination protein $\mathrm{O}$ ) \\
\hline v1.18390 & K03515 & DNA repair protein REV1 \\
\hline v1.04705 & K10991 & DNA repair protein Swi5/Sae3 \\
\hline v1.13920; v1.03800; v1.16133 & K10803 & DNA repair protein XRCC1 \\
\hline v1.15052 & K10879 & DNA repair protein XRCC2 \\
\hline v1.09315 [+ 4 other sequence copies] & K10886 & DNA repair protein $X R C C 4$ \\
\hline v1.02733; v1.24592 & K10868 & DNA repair protein XRS2 \\
\hline v1.14551; v1.23176 & K10873 & DNA repair and recombination protein RAD52 \\
\hline v1.20503 [+ 4 other sequence copies] & K10875 & DNA repair and recombination protein RAD54 \\
\hline v1.23173; v1.16050 & K10877 & DNA repair and recombination protein RAD54B \\
\hline v1.07227; v1.08907; v1.09439; v1.02644 & K10847 & DNA repair protein complementing XP-A cells \\
\hline v1.11534 [+ 5 other sequence copies] & K10865 & Double-strand break repair protein MRE11 \\
\hline v1.07939 & K03660 & N-glycosylase/DNA lyase \\
\hline v1.16163 & K03652 & 3-Methyladenine DNA glycosylase \\
\hline v1.07231 & K10726 & Replicative DNA helicase Mcm \\
\hline v1.05482 & K04499 & RuvB-like protein 1 (pontin 52) \\
\hline v1.19813 & K11338 & RuvB-like protein 2 \\
\hline v1.06890 & K15080 & Single-strand annealing weakened protein 1 \\
\hline v1.17193; v1.14087 & K03111 & Single-strand DNA-binding protein \\
\hline v1.15575 & K10800 & Single-strand monofunctional uracil DNA glycosylase \\
\hline v1.07134 & K10992 & Swi5-dependent recombination DNA repair protein 1 \\
\hline v1.13860 & K03649 & TDG/mug DNA glycosylase family protein \\
\hline v1.14423; v1.14399; v1.05070 & K03648 & Uracil-DNA glycosylase \\
\hline v1.23838 & K10791 & Three prime repair exonuclease 2 \\
\hline v1.19522 & K10839 & UV excision repair protein RAD23 \\
\hline
\end{tabular}

More abundant are the DNA mismatch repair enzymes from the MLH, MSH, Mut and PMS protein families and related glycosylase/lyase proteins for repairing erroneous insertion, deletion and mis-incorporation of bases to arise during DNA replication and recombination. There is additionally a specific gene that encodes a 3 '-endonuclease protein that has a preference to correct mispaired nucleotide sequences. Abundant also are other members of the RAD-family of DNA repair proteins, including 28 sequence copies of a gene encoding the RAD50 protein for DNA double-strand break repair that, together with members of the MRE, Rec, REV, Swi5/Sae3, XRCC and XRS families of recombination and polymerase proteins, have complementary roles in DNA repair. Apparent also in the genome are the DNA helicase proteins, including RuvB-like proteins, which are primarily involved in DNA replication and transcription, but assist also in the repair of DNA damage by separating double strands at affected sites of DNA damage 
to facilitate repair. Of the multiple families of ATPdependent DNA helicase proteins encoded in the coral genome, RecQ and helicase Q predominate. Encoded in the coral genome are 5 homologues of the DNA repair alkB proteins that reverse damage to DNA from alkylation caused by chemical agents by removing methyl groups from 1-methyl adenine and 3-methyl cytosine products in single-stand DNA. Annotated also are genes encoding DNA ligase 3 for repairing single-strand breaks, DNA ligase 4 to repair double-strand breaks, and a DNA cross-link repair $1 \mathrm{C}$ protein with single-strand specific endonuclease activity that may serve in a proofreading function for DNA polymerase. Taken together, expressing this arsenal of DNA protection may provide corals with limited ability to transcribe gene-encoded adaptation to a changing global environment.

\section{Stress response proteins}

Annotation of the A. digitifera genome reveals a wide assortment of thermal shock proteins, molecular chaperones and other stress response elements that are given in (Table 10), excluding antioxidant and redox-protective proteins which are described in the next section. Heat shock proteins $70 \mathrm{kDa}, 90 \mathrm{kDa}, 110 \mathrm{kDA}, \mathrm{HspQ}$ and HspX (the last two proteins being homologues of the bacterial heat shock factor sigma32 and $\alpha$-crystallin, respectively) are encoded in the coral genome, together with several HSP gene transcription factors. HSPs play a role in various cellular functioning such as protein folding, intracellular protein trafficking and resistance to protein denaturation. HSP expression is usually increased on exposure to elevated temperatures and other conditions of biotic and abiotic stress that include infection, inflammation, metabolic hyperactivity, exposure to environmental toxicants, ultraviolet light exposure, starvation, hypoxia and desiccation [243]. HSPs and chaperones are transcriptionally regulated and are induced by heat shock transcription factors [244], of which there are several encoded in the coral genome. Since HSPs are found in virtually all living organisms, it is not surprising that cnidarian $h s p$ transcription and protein expression (HSP60, HSP70 and HSP90) have been profiled as a stress determinant [245-250] and early warning indicator of coral bleaching [251-254]. The coral genome reveals also a cold shock protein encoded by the $\operatorname{csp} A$ gene family, but profiling its expression with other stress response proteins activated by sub-optimum cold temperatures [255] has not been reported. Additionally, the coral genome encodes transcription of a homologue of the universal stress protein A (UspA), a member of an ancient and conserved group of stress-response proteins [256,257], which have been studied mostly in bacteria [258] but have been described also in several plants [259] and animals, including members of the Cnidaria [260]. Usp transcripts have been quantified in the thermal stress response of the coral Montastraea faveolata [261] and its aposymbiotic embryos [262]. Another gene product of potential interest is a homologue of the oxidative-stress responsive protein 1 (OXSR1) that belongs to the Ser/Thr kinase family of proteins, as do other mitogen-stress activated protein kinases (MAPKs), that regulate downstream kinases in response to environmental stress [263] by interacting with the Hsp70 subfamily of proteins [264]. Another significant response protein encoded in the coral genome (Table 10) is a homologue of the stress-induced phosphoprotein 1 (30 domain sequence alignments), known also as the Hsp70Hsp90 organising protein (HOP) belonging to the stress inducible (STI1) family of proteins, which is a principle adaptor protein that mediates the functional cooperation of molecular chaperones Hsp70 and Hsp90 [265,266]. It is yet to be determined if Hop1 transcription may serve as a primary indicator of environmental stress in corals.

Molecular chaperones are a diverse family of proteins expressed by both prokaryotic and eukaryotic organisms that serve to maintain correct protein folding in a 3dimensional functional state, assist in multiprotein complex assembly and protect proteins from irreversible aggregation at synthesis and during conditions of cellular stress [267]. Additionally, heat shock proteins and their co-chaperones may regulate cell death pathways by inhibition of apoptosis [268]. The coral genome encodes a large number of DnaJ subfamily (J-domain) chaperones (Hsp40) that with co-chaperone GrpE (Table 10) regulates the ATPase activity of Hsp70 (DnaK in bacteria) to enable correct protein folding [269]. The coral genome encodes homologues of the molecular chaperones HscA (specialised Hsp70), the redox-regulated chaperone Hsp33, HtpG (high temperature protein G), members of the calnexin/calreticulin chaperone system of the endoplasmic reticulum, a mitochondrial chaperone BCS1 protein necessary for the assembly of the respiratory chain complex III and a specific chaperone of trimethyl $\mathrm{N}$-oxide reductase (TorA). The coral genome also encodes hypoxia-inducible factors (HIFs) that moderate the deleterious effects of hypoxia on cellular metabolism (reviewed in [270]). In the HIF signalling cascade, the alpha subunits of HIF are hydroxylated at conserved proline residues by HIF prolyl-hydroxylases allowing their recognition for proteasomal degradation, which occurs during normoxic conditions but is repressed by oxygen depletion. Hypoxiastabilised HIF1 upregulates the expression of enzymes principally of the oxygen-independent glycolysis pathway, and in higher animals promotes vascularisation, whereas the mammalian HIF2 paralogue regulates erythropoietin control of hepatic erythrocyte production in response to hypoxic stress [271]. The roles of HIF1 and HIF2 homologues in corals have been established, with HIF1 regulation of glycolysis critical to metabolic function during the dark diurnal anoxic state of coral respiration [193,272]. 
Table 10 Stress response proteins in the predicted proteome of $A$. digitifera

\begin{tabular}{|c|c|c|}
\hline Gene sequence & KEGG Orthology & Encoded protein description \\
\hline v1.04616; v1.06277 & K03694 & ATP-dependent Clp protease subunit ClpA \\
\hline v1.04617; v1.23486; v1.23484; v1.10207 & K03695 & ATP-dependent Clp protease subunit ClpB \\
\hline v1.13464 & K03697 & ATP-dependent Clp protease subunit ClpE \\
\hline v1.06903; v1.11461 & K06891 & ATP-dependent Clp protease adaptor protein ClpS \\
\hline v1.12577; v1.09531; v1.17184 & K03544 & ATP-dependent Clp protease subunit ClpX \\
\hline v1.09407 & K08054 & Calnexin (protein-folding chaperone) \\
\hline v1.16781 & K08057 & Calreticulin $\left(\mathrm{Ca}^{2+}\right.$-binding chaperone) \\
\hline v1.04005 & K10098 & Calreticulin $3\left(\mathrm{Ca}^{2+}\right.$-binding chaperone) \\
\hline v1.02702[+ 5 other sequence copies] & K03704 & Cold shock protein (beta-ribbon, CspA family) \\
\hline v1.01907; v1.18998 & K07213 & Copper chaperone \\
\hline v1.23457; v1.01713; v1.19228 & K04569 & Copper chaperone for superoxide dismutase \\
\hline v1.08719; v1.19128 & K09502 & DnaJ homologue subfamily A member 1 \\
\hline v1.08719; v1.18432 & K09503 & DnaJ homologue subfamily A member 2 \\
\hline v1.16210; v1.22054 & K09504 & DnaJ homologue subfamily A member 3 \\
\hline v1.19128 & K09505 & DnaJ homologue subfamily A member 4 \\
\hline v1.04818 [+ 6 other sequence copies] & K09506 & DnaJ homologue subfamily A member 5 \\
\hline v1.02841; v1.02842 & K09507 & DnaJ homologue subfamily B member 1 \\
\hline v1.00368; v1.13308; v1.16977; v1.03340 & K09508 & DnaJ homologue subfamily B member 2 \\
\hline v1.11537; v1.09205; v1.08628; v1.02840 & K09511 & DnaJ homologue subfamily B member 5 \\
\hline v1.24549 [+ 9 other sequence copies] & K09512 & DnaJ homologue subfamily B member 6 \\
\hline v1.01573 & K09513 & DnaJ homologue subfamily B member 7 \\
\hline v1.00352; v1.09196; v1.06645 & K09514 & DnaJ homologue subfamily B member 8 \\
\hline v1.18536 [+ 4 other sequence copies] & K09515 & DnaJ homologue subfamily B member 9 \\
\hline v1.14710 & K09517 & DnaJ homologue subfamily B member 11 \\
\hline v1.14959 & K09518 & DnaJ homologue subfamily B member 12 \\
\hline v1.09205 & K09519 & DnaJ homologue subfamily B member 13 \\
\hline v1.16242 & K09520 & DnaJ homologue subfamily B member 14 \\
\hline v1.20109; v1.03468 & K09521 & DnaJ homologue subfamily $\mathrm{C}$ member 1 \\
\hline v1.07111 [+ 5 other sequence copies] & K09522 & DnaJ homologue subfamily $\mathrm{C}$ member 2 \\
\hline v1.21077 [+ 13 other sequence copies] & K09523 & DnaJ homologue subfamily $\mathrm{C}$ member 3 \\
\hline v1.07739; v1.22910 & K09524 & DnaJ homologue subfamily $\mathrm{C}$ member 4 \\
\hline v1.01239 [+ 13 other sequence copies] & K09525 & DnaJ homologue subfamily $\mathrm{C}$ member 5 \\
\hline v1.17629 [+ 29 other sequence copies] & K09527 & DnaJ homologue subfamily $C$ member 7 \\
\hline v1.18619; v1.08300; v1.23789 & K09528 & DnaJ homologue subfamily $\mathrm{C}$ member 8 \\
\hline v1.13575; v1.04213 & K09529 & DnaJ homologue subfamily $\mathrm{C}$ member 9 \\
\hline v1.05956; v1.05955; v1.21265; v1.21205 & K09530 & DnaJ homologue subfamily $\mathrm{C}$ member 10 \\
\hline v1.13525; v1.04120 & K09531 & DnaJ homologue subfamily $\mathrm{C}$ member 11 \\
\hline v1.09496 [+ 4 other sequence copies] & K09533 & DnaJ homologue subfamily $\mathrm{C}$ member 13 \\
\hline v1.24546 & K09534 & DnaJ homologue subfamily $\mathrm{C}$ member 14 \\
\hline v1.05866 & K09536 & DnaJ homologue subfamily $\mathrm{C}$ member 16 \\
\hline v1.16151; v1.08307; v1.14980 & K09537 & DnaJ homologue subfamily $\mathrm{C}$ member 17 \\
\hline v1.16309 & K09539 & DnaJ homologue subfamily $\mathrm{C}$ member 19 \\
\hline v1.05241; v1.22999; v1.17372 & K14258 & Facilitated trehalose transporter (anhydrobiosis) \\
\hline v1.12967; v1.19789 & K14590 & FtsJ methyltransferase [heat shock protein] \\
\hline
\end{tabular}


Table 10 Stress response proteins in the predicted proteome of $\boldsymbol{A}$. digitifera (Continued)

\begin{tabular}{|c|c|c|}
\hline v1.02247 & K09414 & Heat shock transcription factor 1 \\
\hline v1.24112 & K09416 & Heat shock transcription factor 3 \\
\hline v1.05839 & K09419 & Heat shock transcription factor, other eukaryote \\
\hline v1.12890 [+ 10 other sequence copies] & K03283 & Heat shock $70 \mathrm{kDa}$ protein 1/8 \\
\hline v1.07996 & K09489 & Heat shock 70 kDa protein 4 \\
\hline v1.02854; v1.07452; v1.01623 & K09490 & Heat shock 70 kDa protein 5 \\
\hline v1.14149; v1.14150 & K09487 & Heat shock protein $90 \mathrm{kDa}$ beta \\
\hline v1.07995; v1.07996; v1.16399; v1.11283 & K09485 & Heat shock protein 110 kDa \\
\hline v1.08943; v1.05577 & K11940 & Heat shock protein HspQ \\
\hline v1.00537; v1.00043 & K03799 & Heat shock protein $\mathrm{HtpX}$ \\
\hline v1.01623 & K04046 & Hypothetical chaperone protein \\
\hline v1.16216 & K08268 & Hypoxia-inducible factor 1 alpha \\
\hline v1.08869; v1.15120 & K09097 & Hypoxia-inducible factor 1 beta \\
\hline v1.22724 & K09095 & Hypoxia-inducible factor 2 alpha \\
\hline v1.23698 [+ 16 other sequence copies] & K06711 & Hypoxia-inducible factor prolyl 4-hydroxylase \\
\hline v1.16737; v1.22345 & K09486 & Hypoxia up-regulated 1 (heat shock protein 70 family) \\
\hline v1.10188 & K08900 & Mitochondrial chaperone BCS1 \\
\hline v1.17197; v1.04394 & K04445 & Mitogen-stress activated protein kinases \\
\hline v1.16301; v1.21224; v1.19344 & K04043 & Molecular chaperone DnaK \\
\hline v1.09682; v1.16748; v1.07471; v1.13624 & K03687 & Molecular chaperone GrpE \\
\hline v1.01621; v1.04945; v1.15919 & K04044 & Molecular chaperone HscA \\
\hline v1.18210 & K04083 & Molecular chaperone Hsp33 \\
\hline v1.17478; v1.16977; v1.10289; v1.19907 & K04079 & Molecular chaperone HtpG \\
\hline v1.08895; v1.18099 & K11416 & Mono-ADP-ribosyltransferase sirtuin 6 \\
\hline v1.02024 & K11411 & NAD-dependent deacetylase sirtuin 1 \\
\hline v1.04813 & K11412 & NAD-dependent deacetylase sirtuin 2 \\
\hline v1.22049; v1.22211; v1.02221 & K11413 & NAD-dependent deacetylase sirtuin 3 \\
\hline v1.11849; v1.02221 & K11414 & NAD-dependent deacetylase sirtuin 4 \\
\hline v1.05495 & K11415 & NAD-dependent deacetylase sirtuin 5 \\
\hline v1.04868 & K11417 & NAD-dependent deacetylase sirtuin 7 \\
\hline v1.15070 [+ 4 other sequence copies] & K08835 & Oxidative-stress responsive protein 1 (OXSR1) \\
\hline v1.04503 & K11875 & Proteasome assembly chaperone 1 \\
\hline v1.01531 & K11878 & Proteasome assembly chaperone 4 \\
\hline v1.01210 & K11879 & Proteasome chaperone 1 \\
\hline v1.18611 & K11880 & Proteasome chaperone 2 \\
\hline v1.00599 [+ 29 other sequence copies] & K09553 & Stress-induced-phosphoprotein 1 (HOP1) \\
\hline v1.08830 & K13057 & Trehalose synthase (anhydrobiosis) \\
\hline v1.22042 & K03533 & TorA specific chaperone \\
\hline v1.16986 [+ 7 other sequence copies] & K06149 & Universal stress protein A \\
\hline
\end{tabular}

Heat shock proteins that repair unfolded or misfolded protein have a complementary function to the ubiquitinproteasome system (ubiquitins not tabulated) that selects damaged protein for degradation [273], such that HSP chaperones and the proteasome act jointly to preserve cellular proteostasis [274,275]. Thus, several proteasome chaperones and assembly chaperones are encoded in the A. digitifera genome (Table 10). While proteasome chaperones serve to target aberrant proteins for ubiquination, the proteasome chaperones facilitates $20 \mathrm{~S}$ assembly for 
biogenesis of the multiunit $26 \mathrm{~S}$ proteasome that is activated in response to stress [276,277], possibly by FtsJ (aka RrmJ), a well-conserved heat shock protein having novel ribosomal methyltransferase activity that targets methylation of 26S rRNA under heat shock control [278,279]. The HspQ protein encoded in the coral genome, although studied almost exclusively in bacteria, is known to stimulate degradation of denatured proteins caused by hyperthermal stress, particularly DnaA that initiates DNA replication in prokaryotes [280]. Specifically, HspQ (heat shock factor sigma32) regulates the expression of Clp ATPase-dependent protease family enzymes [281,282], of which ClpA, ClpB, ClipE, the protease adaptor protein ClpS [283] and the unfoldase ClpX protein [284] are encoded in the coral genome (Table 10). HspX is a small $16 \mathrm{kDa} \alpha$-crystallin chaperone (Acr) protein belonging to the Hsp20 family of proteins [285] that suppresses thermal denaturation and aggregation of proteins [285]. Significantly, Acr proteins are known to bind with carbonic anhydrase [286] and may have importance in moderating stress-induced loss of calcium deposition. Thus, HspX/ Acr expression may account for differences in the thermal sensitivity of corals to calcification that varies among genera [287]. In a different context, HspX is attracting considerable attention for its potential to elicit long-term protective immunity against human Mycobacterium tuberculosis infection by chaperoning a host-protective antigen [288] that by extension, but yet untested, may likewise repress virulence in the initiation and progression of microbial coral disease $[289,290]$.

The coral genome encodes complete membership of the human sirtuin (SIRT1-7) family of $\mathrm{NAD}(+)$-dependent protein deacetylases and ADP-ribosyltransferases. Mammalian SIRT1 (a homologue of yeast Sir2) is an important regulator of metabolism, cell differentiation, stress response transcription and pathways of cellular senescence (reviewed in [291]). SIRT proteins regulate chromatin function through deacetylation of histones that promote subsequent alterations in the methylation of histones and DNA to affect, via deactivation of nuclear transcription factors and co-regulators, epigenetic control of nuclear transcription. As $\mathrm{NAD}^{+}$-dependent enzymes, SIRT1 can regulate gene expression in response to cellular $\mathrm{NAD}^{+}$/ NADH redox status providing a metabolic template for epigenetic transcriptome reprogramming [292,293]. In the human genome repertoire, SIRT1 modulates cellular responses to hypoxia by deacetylation of HIF1 $\alpha$ [294] and inhibits nitric oxide synthesis by suppression of the nuclear factor-kappaB (NF-kB) signalling pathway [295], SIRT2 promotes oxidative stress resistance by deacetylation of forkhead box O (FOXO) proteins [296], SIRT3 decreases ROS production in adipocytes [297], SIRT4 regulates fatty acid metabolism and stressresponse elements of mitochondrial gene expression
[298], SIRT5 is a protein lysine desuccinylase and demalonylase of unknown function [299], SIRT6 activates base-excision repair [300] and SIRT7 inhibits apoptosis induced by oxidative stress by deacylation of p53 [301,302]. The significance of coral SIRT proteins, by analogy, to exert stress tolerance is yet to be examined.

Metallochaperones are an important class of enzymes that transport co-factor metal ions to specific proteins [303]. The copper chaperone protein ATX1 (human ATOX1) delivers cytosolic copper to $\mathrm{Cu}$-ATPase proteins and serves as a metal homeostasis factor to prevent Fenton-type production of highly reactive hydroxyl radicals. ATX1, which is strongly induced by molecular oxygen, functions additionally as an antioxidant to protect cells against the toxicity of both the superoxide anion and hydrogen peroxide [304]. Encoded also is a specific copper chaperone essential to the activation of $\mathrm{Cu} / \mathrm{Zn}$ superoxide dismutase $[305,306]$ that is enhanced by photooxidative stress in scleractinian corals [307], although reported to be less pronounced in the host than in symbiotic algae [308]. In addition to high light exposure, reef-building corals of shallow reef flats are occasionally exposed to the atmosphere for periods that can last several hours during extreme low tides. Hence, species that are adapted to withstand acute desiccation (anhydrobiosis) have a better chance of surviving such conditions. The disaccharide trehalose is an osmolyte that in some plants and animals allows them to survive prolonged periods of desiccation [309]. The hydrated sugar has high water retention that forms a gel phase when cells dehydrate, which on rehydration allows normal cellular activity to resume without damage that would otherwise follow a dehydration/rehydration cycle. Furthermore, trehalose is highly effective in protecting enzymes in their native state from inactivation from thermal denaturation [310]. Given that A digitifera is endemic on shallow reef flats prone to exposure at low tides [311], it is not surprising that the coral genome encodes trehalose synthase and a facilitated trehalose transporter for protection against dehydration.

\section{Antioxidant and redox-protective proteins}

Oxygen is vital for life, but it can also cause damage to cells, particularly at elevated levels. In coral symbiosis, the photosynthetic endosymbionts of corals typically produce more oxygen than the holobiont is able to consume by respiration, so that coral tissues are hyperoxic with tissue $\mathrm{pO}_{2}$ levels often exceeding $250 \%$ of air saturation during daylight illumination [193]. Furthermore, because algal symbionts reside within the endodermal cells of their host, coral tissues must be transparent to facilitate the penetration of downwelling light required for photosynthesis by their algal consorts. In clear shallow waters this entails concurrent exposure to vulnerable 
molecular sites of both partners to damaging wavelengths of ultraviolet radiation. The synergistic effects of tissue hyperoxia and UV exposure can cause oxidative damage to the symbiosis via the photochemical production of cytotoxic oxygen species [312] that are produced also during normal mitochondrial function [313]. Consequently, protective proteins (antioxidant enzymes) are expressed to maintain the fine balance between oxygen metabolism and the production of potentially toxic reactive oxygen species (ROS). If this balance is not maintained by regulation of oxidative and reductive processes (redox regulation), oxidative stress occurs by the generation of excess ROS, causing damage to DNA, proteins, and lipids. Corals elaborate a variety of molecular defences that including the production of UV-protective sunscreens (MAAS), antioxidants, antioxidant enzymes, chaperones and heat shock proteins, which are often inducible under conditions of enhanced oxidative stress [307], including conditions that elicit coral bleaching $[314,315]$. An excellent review on the formation of ROS and the role of antioxidants and antioxidant enzymes in the field of redox biology is given by Halliwell [316].

Annotation of the $A$. digitifera genome reveals sequences encoding two isoforms of the antioxidant enzyme superoxide dismutase (SOD) from both the $\mathrm{Cu} / \mathrm{Zn}$ and $\mathrm{Fe} / \mathrm{Mn}$ families of SOD (Table 11). These metalloprotein enzymes catalyse the dismutation of superoxide to yield molecular oxygen and hydrogen peroxide, the latter being less harmful than superoxide. Superoxide can oxidize proteins, denature enzymes, oxidize lipids and fragment DNA. By removing superoxide, SOD protects also against the production of reactive peroxynitrite formed by the combination of superoxide and nitric oxide, which is a precursor reactant for production of the supra-reactive hydroxyl radical. Hydrogen peroxide per se is a mild oxidant, but it readily oxidises free cellular ferrous iron to ferric iron with production of hydroxyl radicals via the Fenton reaction. Accordingly, both the removal of hydrogen peroxide and the expression of proteins, such as transferrin, (bacterio)ferritins and metallothioneins, that bind reactive (transition) metal ions is important to protect cellular components from acute oxidative damage. Oddly, only a metallothionein expression activator was found encoded in the coral genome without finding a sequence to activate transcription of the actual metallothionein protein gene.

As expected from the foregoing, the genome of $A$. digitifera encodes the antioxidant enzyme catalase (CAT) that is highly efficient in decomposing hydrogen peroxide to yield molecular oxygen and water. Two isoforms of CAT are encoded at multiple sites. One is a peroxisomal eukaryotic CAT enzyme that targets the removal of hydrogen peroxide formed as a by-product of oxidase enzymes, and the other is a related catalase domain-containing protein presumed also to decompose hydrogen peroxide.
Glutathione peroxidise (GPx) reduces both hydrogen peroxide and lipid hydroperoxides, the latter of which are formed by radical-induced lipid autoxidation. Phototrophic organisms, including higher plants, utilise ascorbate peroxidase (APx) as a primary catalyst for the reduction of hydrogen peroxide and lipid hydroperoxides. However, unlike the freshwater cnidarian $H$. viridis [164], there is no evidence for transfer of APx-encoding genes to A. digitifera. The antioxidant enzymes SOD, CAT, GPx and APx are well characterised in the algal and animal partners of coral symbiosis (reviewed in [317]). Additionally, the coral genome has sequences encoding alkyl hydroperoxide reductase, hydroperoxide lyase, phospholipidhydroperoxide glutathione peroxidase, thiol peroxidase and multiple isoforms of peroxiredoxin, all of which function in the detoxification of organo-hydroperoxides that are produced as a by-product of aerobic metabolism. Additionally, sulfiredoxin (Table 11) repairs peroxiredoxins when these enzymes are inhibited by over-oxidation [318].

Thioredoxins and glutaredoxins have important secondary roles in regulating multiple pathways in many biological processes, including redox signalling of apoptotic pathways, which have been attributed to processes involved in coral bleaching [56]. Other enzymes that regulate cellular thiol-disulfide homeostasis in this coral are monothiol glutaredoxin and protein-disulfide reductase. The coral genome encodes the ubiquitous thioredoxin system of antioxidant proteins (Table 11) that act as electron donors to peroxidases and ribonucleotide reductase (the latter not tabulated). By cysteine thiol-disulfide exchange, thioredoxins function as a protein thiol-disulfide oxidoreductase [319]. In the thioredoxin system, thioredoxins are maintained in their reduced state by NADPH-dependent, flavoenzyme thioredoxin reductase [320]. Peptide-methionine (R)-S-oxide reductase can additionally rescue thioredoxin from oxidative inactivation by disulfide reduction. Related glutaredoxins share many of the functions of thioredoxins but are reduced directly by glutathione, rather than by a specific reducing enzyme, while in turn glutathione is kept in its native state by NADPH: glutathione reductase.

In recent years there has been a particular focus on the role of ROS in coral bleaching, fuelled by dire prediction of future catastrophic episodes caused by environmental change affected by global warming [321]. Early predictions of coral bleaching were based principally on physical environmental parameters, rather than on the determination of the physiological state of coral populations to such conditions. While gene expression markers are being developed to monitor sub-bleaching levels of stress in situ (e.g., [261]), Kenkel et al. [322] opined that the current challenge for implementing expression-based methods lies in identifying coral genes demonstrating the most pronounced and consistent 
Table 11 Antioxidant and redox-protective proteins in the predicted proteome of $A$. digitifera

\begin{tabular}{|c|c|c|}
\hline Gene sequence & KEGG Orthology & Encoded protein description \\
\hline v1.10918 & K04756 & Alkyl hydroperoxide reductase subunit D \\
\hline v1.11551 & K03387 & Alkyl hydroperoxide reductase subunit F \\
\hline v1.07812 & K03594 & Bacterioferritin \\
\hline v1.21362 [+ 4 other sequence copies] & K00429 & Catalase (bacterial) \\
\hline v1.17525 [+ 4 other sequence copies] & K03781 & Catalase (peroxisonal) \\
\hline v1.23457; v1.01713; v1.19228 & K04569 & Copper chaperone for superoxide dismutase \\
\hline v1.20153; v1.20154 & K10528 & Hydroperoxide lyase \\
\hline v1.19687; v1.19688; v1.18796; v1.18795 & K00522 & Ferritin heavy chain \\
\hline v1.06441 & K03674 & Glutaredoxin 1 \\
\hline v1.19449 & K03675 & Glutaredoxin 2 \\
\hline v1.14929 [+ 5 other sequence copies] & K03676 & Glutaredoxin 3 \\
\hline v1.13285; v1.03722; v1.03688; v1.10496 & K00432 & Glutathione peroxidase \\
\hline v1.13174; v1.13775; v1.05473 & K00383 & Glutathione reductase (NADPH) \\
\hline v1.14344; v1.19399; v1.01421 & K01920 & Glutathione synthase \\
\hline v1.02173 & K09238 & Metallothionein expression activator \\
\hline v1.09719; v1.16134; v1.18608 & K07390 & Monothiol glutaredoxin \\
\hline v1.14890; v1.17685 & K07305 & Peptide-methionine (R)-S-oxide reductase \\
\hline v1.14909 & K00435 & Peroxiredoxin \\
\hline v1.14106 & K13279 & Peroxiredoxin 1 \\
\hline v1.08691 & K11187 & Peroxiredoxin 5, atypical 2-Cys peroxiredoxin \\
\hline v1.01410 & K11188 & Peroxiredoxin 6, 1-Cys peroxiredoxin \\
\hline v1.03688 & K05361 & Phospholipid-hydroperoxide glutathione peroxidase \\
\hline v1.05148 & K05905 & Protein-disulfide reductase \\
\hline v1.02922; v1.22772; v1.24164 & K05360 & Protein-disulfide reductase (glutathione) \\
\hline v1.06810 & K12260 & Sulfiredoxin \\
\hline v1.01713 [+ 4 other sequence copies] & K04565 & Superoxide dismutase, Cu/Zn family \\
\hline v1.09974; v1.20324 & K04564 & Superoxide dismutase, Fe/Mn family \\
\hline v1.02378 & K11065 & Thiol peroxidase, atypical 2-Cys peroxiredoxin \\
\hline v1.22324 [+ 7 other sequence copies] & K03671 & Thioredoxin 1 \\
\hline v1.05148; v1.03230; v1.20699 & K03672 & Thioredoxin 2 \\
\hline v1.17881 [+ 5 other sequence copies] & K13984 & Thioredoxin domain-containing protein 5 \\
\hline v1.04532; v1.24501 & K09585 & Thioredoxin domain-containing protein 10 \\
\hline v1.11551; v1.19049 & K00384 & Thioredoxin reductase (NADPH) \\
\hline v1.10930 & K14736 & Transferrin \\
\hline
\end{tabular}

stress response, preferably with a large dynamic range to enable reliable quantification. To this end, we offer in Table 11 the annotation of novel redox-related genes for examination as potential candidate biomarkers to monitor the physiological response of $A$. digitifera to environmental stress.

\section{Proteins of cellular apoptosis}

Apoptosis is the signalling of programmed cell death (PCD) that occurs in multicellular organisms in response to cellular injury. A key feature of apoptosis is the activation of endogenous endonucleases causing nuclear fragmentation, chromatin condensation and chromosomal DNA fragmentation, which typically presents in affected cells by the morphological appearance of plasma membrane blebbing and cell shrinkage. Caspases and related family member proteases are described as "executioners" of apoptosis that on post-translational activation degrade the regulatory proteins that prevent DNA degradation. Fragmentation of nuclear DNA is one of the 
hallmarks of apoptotic cell death that occurs by PCD stimuli in a wide variety of proliferating cells. NF- $\mathrm{KB}$ is a protein complex that controls the transcription of DNA that can induce the expression of nitric oxide synthesis (NOS) to produce NO that is a well-known promoter of the of the pro-apoptotic transcription factor p53 cellcycle gatekeeper of the caspase cascade. In contrast to necrosis, which is the outcome of PCD, apoptosis mediates the fragmentation of damaged cells, which by phagocytosis are removed or degraded in phagolysosomes to spare surviving cells from the uncontrolled release of cytotoxic agents. Proteins of the caspasemediated apoptotic cascade are regarded as products of constituent housekeeping genes that are necessary to maintain healthy multicellular function [323]. In the progression of cnidarian bleaching, apoptotic pathways are activated [322-325], but not all corals that suffer bleaching are destined to die [326,327]. Coral survival has been attributed to having a high level of apoptotic protection at the onset of coral bleaching [328] and during post-bleaching recovery [329] by specific activation of anti-apoptotic $\mathrm{Bcl}-2$ proteins in surviving cells [330].

Cnidarians have a complex apoptotic protein network that has exceptional ancestral complexity and is comparable to that of higher vertebrates [331,332]. Cnidarian metamorphosis is tightly coupled with caspase-dependent apoptosis [333] and subsequent host-symbiont selection by post-phagocytic winnowing of Symbiodinium genotypes during the establishment of coral-dinoflagellate mutualism [334]. As expected, the coral genome of $A$. digitifera encodes multiple isoforms of genes that transcribe the caspase family of apoptotic effectors (Table 12). Included in this signalling pathway are the pro- and antiapoptotic Bax/Bcl regulators and Bcl-2 athanogene (DNAbinding) activators of apoptosis. Notable in our annotation dataset are multiple genes that encode the protein domains of the apoptotic protease-activating factor (Apaf) that triggers assembly of the apoptosome leading to caspase activation [335]. Additional to this arsenal of cell cycle regulators are the death associated protein- 6 (DAXX), a Fas-binding adaptor of c-Jun $\mathrm{N}$-terminal kinase (JNK) activation [336], death-associated protein kinase (DAPK), a mediator of calcium/calmodulin-regulated Ser/ Thr kinase [337], and the programmed cell death 6-inter acting protein (PDCD6IP), which binds to PDCD-6 for execution of apoptosis via the caspase-3 pathway [338]. PDCD6IP activation of apoptosis is an enigma since PDCD-6 is not encoded in the coral genome, nor is caspase-3. Other cell cycle regulators are the p53 binding and p53-associated parkin-like proteins, and the activating TP53 regulating kinase protein and TP53 apoptosis effector of TP53 gene expression.

Our genome annotation reveals 73 sequence matches for expressing the Apaf protein domain that, in conjunction with a high copy number for expressing caspase-8 (28 protein sequence matches), may enhance coral survival during embryogenesis by suppressing receptor-induced protein kinase (45 sequence matches) during early development [339]. The most conserved function of the CAPS2/RIPK adaptor (45 sequence matches) encoded in the coral genome is its essential regulation of apoptosis [340]. We find a wide repertoire of genes that additionally encode proteins that mediate apoptosis (Table 12). Amongst these are the calpain $\mathrm{Ca}^{+2}$-sensing family of proteins that initiate the signalling of apoptotic pathways [341]. There are 79 matches to sequences that encode the tumor necrosis Fas superfamily member 6 (TNFRSF6) receptor, which coupled with the death domain (FADD) protein is a cell signalling mediator for recruitment of caspase- 8 that activates the apoptotic cysteine protease cascade. Coincident in the genome are 67 sequences encoding the leucine-rich repeat and death domain-containing (LRDD) adaptor that, by interacting with other p53-inducible death domain-containing (PIDD) proteins such as FADD, induces the caspase-2 pathway of apoptosis in response to DNA damage [342]. Elements of the NF- $\mathrm{kB}$ signalling pathway of cnidarians are highly conserved traits [343], which includes the caspase cascade and the pro-apoptotic and anti-apoptotic Bcl-2 family of proteins [344]. The coral genome of $A$. digitifera encodes the pleiotropic nuclear factor NF-kB p105 subunit, and astonishingly there are 212 sequence matches to the NF- $\mathrm{kB}$ inhibitor-like protein 2 domain with fewer matches to the NF- $\mathrm{kB}$ inhibitor-like protein 1 and NF- $\mathrm{kB}$ family inhibitors alpha, delta and epsilon. Evident in our genome annotation is the tumor necrosis factor-alpha induced protein 3 (TNFAIP3), a cytokine produced by activated (inflammatory) macrophages. Although TNF cytokines are a major extrinsic mediator of cellular apoptotic pathways, the precise function of the superfamily members of TNF ligands and receptors (Table 12) remains elusive in coral symbiology.

\section{Microbial symbiosis and pathogenicity}

It is well established that corals associate with a vast consortia of microbes, including phototrophic symbionts (Symbiodinium spp.) and other eukaryotic microbionts, cyanophytes, heterotrophic bacteria, archaea and viruses [345]. Corals harbour diverse and abundant prokaryotic communities with distinct populations residing in separate habitats of the host skeleton, tissues and surface mucus layer (reviewed in [203]). Microbial populations are dominated by a few coral-specific taxonomic traits [346], but the majority of the population comprises a high number of taxonomically diverse, low-abundance ribotypes [347] with much of the diversity within the coral microbiome belonging to the "rare" biosphere [348,349]. The coral microbiome is vital to the nutrition and health of the holobiont [350] and contributes 
Table 12 Proteins of cellular apoptosis in the predicted proteome of $A$. digitifera

\begin{tabular}{|c|c|c|}
\hline Gene sequence & KEGG Orthology & Encoded protein description \\
\hline v1.17521; v1.02505; v1.20702; v1.05077 & K02159 & Apoptosis regulator BAX (BCL2-associated) \\
\hline v1.05086; v1.20659 & K02161 & Apoptosis regulator $\mathrm{BCL}-2$ \\
\hline v1.17522; v1.00181; v1.10817; v1.20703 & K02163 & Apoptosis regulator $\mathrm{BCL}-\mathrm{W}$ \\
\hline v1.05147 [+ 6 other sequence copies] & K12875 & Apoptotic chromatin condensation inducer \\
\hline v1.22264 [+ 72 other sequence copies] & K02084 & Apoptotic protease-activating factor (Apaf) \\
\hline v1.17326; v1.20305; v1.11586 & K09555 & BCL2-associated athanogene 1 \\
\hline v1.08601 & K09558 & BCL2-associated athanogene 4 \\
\hline v1.02839 & K09559 & BCL2-associated athanogene 5 \\
\hline v1.01518 & K13087 & BCL2-associated transcription factor 1 \\
\hline v1.20278; v1.00172; v1.07858 & K14021 & BCL-2 homologueous antagonist/killer \\
\hline v1.09624 & K02561 & BCL2-related (ovarian) killer protein \\
\hline v1.17749 & K08573 & Calpain-3 \\
\hline v1.00595; v1.14671; v1.00040 & K08574 & Calpain-5 \\
\hline v1.00040 & K08575 & Calpain-6 \\
\hline v1.19153; v1.17749 & K08576 & Calpain-7 \\
\hline v1.15226 & K04740 & Calpain-12 \\
\hline v1.02951 & K08582 & Calpain-15 \\
\hline v1.11167; v1.06681; v1.20230; v1.01376 & K08585 & Calpain, invertebrate \\
\hline v1.0312 7 [+ 6 other sequence copies] & K08583 & Calpain, small subunit 1 \\
\hline v1.17229; v1.00023; v1.09976 & K02186 & Caspase 2 \\
\hline v1.11989 [+ 5 other sequence copies] & K04397 & Caspase 7 \\
\hline v1.02756 [+ 27 other sequence copies] & K04398 & Caspase 8 \\
\hline v1.01818 & K04399 & Caspase 9 \\
\hline v1.00817 [+ 4 other sequence copies] & K04400 & Caspase 10 \\
\hline v1.02005 & K04741 & Caspase 12 \\
\hline v1.00818 [+ 11 other sequence copies] & K04489 & Caspase apoptosis-related cysteine protease \\
\hline v1.13260 & K07367 & Caspase recruitment domain-containing protein 11 \\
\hline v1.06297 [+ 44 other sequence copies] & K02832 & CASP2 and RIPK1 adaptor with death domain \\
\hline v1.21531 & K02308 & Death-associated protein 6 (DAXX) \\
\hline v1.09448; v1.15529; v1.20164 & K08803 & Death-associated protein kinase (DAPK) \\
\hline v1.23110; v1.14222; v1.03658 & K12366 & Engulfment and motility protein 1 (phagocytosis/apoptosis) \\
\hline v1.18448 [+ 78 other sequence copies] & K02373 & Fas (TNFRSF6)-associated via death domain (FADD) \\
\hline v1.24288 [+ 66 other sequence copies] & K10130 & Leucine-rich repeats and death domain-containing protein \\
\hline v1.20620 & K04734 & NF-kappa-B inhibitor alpha \\
\hline v1.01706 & K14214 & NF-kappa-B inhibitor delta \\
\hline v1.10378; v1.10729; 1.05609; v1.05609 & K05872 & NF-kappa-B inhibitor epsilon \\
\hline v1.17893; v1.22419; v1.00700; v1.08415 & K09256 & NF-kappa-B inhibitor-like protein 1 \\
\hline v1.04158 [+ 211 other sequence copies] & K09257 & NF-kappa-B inhibitor-like protein 2 \\
\hline v1.05320; v1.06979; v1.04467; v1.21371 & K02580 & Nuclear factor NF-kappa-B p105 subunit \\
\hline v1.20334; v1.22743 & K11970 & p53-Associated parkin-like cytoplasmic protein \\
\hline v1.14920; v1.11864; v1.15271; v1.11865 & K06643 & p53-Binding protein \\
\hline v1.04289 & K06708 & Programmed cell death 1 ligand 2 \\
\hline v1.05882 [+ 7 other sequence copies] & K12200 & Programmed cell death 6-interacting protein (PDCD6!P) \\
\hline v1.10959; v1.04994 & K04727 & Programmed cell death 8 apoptosis-inducing factor \\
\hline
\end{tabular}




\section{Table 12 Proteins of cellular apoptosis in the predicted proteome of $\boldsymbol{A}$. digitifera (Continued)}

\begin{tabular}{|c|c|c|}
\hline v1.16714 & K06875 & Programmed cell death protein 5 (PDCD-5) \\
\hline V1.13112 & K03171 & Tnfrsf1a-associated via death domain \\
\hline v1.24655; v1.12385 & K10136 & TP53 apoptosis effector \\
\hline v1.09087 & K08851 & TP53 regulating kinase \\
\hline v1.05030; v1.07044 & K11859 & Tumor necrosis factor, alpha-induced protein 3 \\
\hline v1.22799 & K04389 & Tumor necrosis factor ligand superfamily member 6 \\
\hline v1.05776 & K05470 & Tumor necrosis factor ligand superfamily member 7 \\
\hline v1.13754 & K05472 & Tumor necrosis factor ligand superfamily member 9 \\
\hline v1.21776 [+ 6 other sequence copies] & K04721 & Tumor necrosis factor ligand superfamily member 10 \\
\hline v1.04001 & K05473 & Tumor necrosis factor ligand superfamily member 11 \\
\hline v1.19776 & K05474 & Tumor necrosis factor ligand superfamily member 12 \\
\hline v1.09015; v1.14041 & K03158 & Tumor necrosis factor receptor superfamily member $1 \mathrm{~A}$ \\
\hline v1.07010 & K05141 & Tumor necrosis factor receptor superfamily member $1 \mathrm{~B}$ \\
\hline v1.19735 & K05142 & Tumor necrosis factor receptor superfamily member 4 \\
\hline V1.13754 & K03160 & Tumor necrosis factor receptor superfamily member 5 \\
\hline v1.22577 & K05143 & Tumor necrosis factor receptor superfamily member $6 \mathrm{~B}$ \\
\hline v1.20003 & K05144 & Tumor necrosis factor receptor superfamily member 7 \\
\hline v1.23750; v1.17970; v1.19022 & K05146 & Tumor necrosis factor receptor superfamily member 9 \\
\hline v1.07527 & K05148 & Tumor necrosis factor receptor superfamily member $11 \mathrm{~B}$ \\
\hline v1.10221 & K05151 & Tumor necrosis factor receptor superfamily member $13 \mathrm{C}$ \\
\hline v1.14826; v1.01054 & K05152 & Tumor necrosis factor receptor superfamily member 14 \\
\hline v1.09514 & K05156 & Tumor necrosis factor receptor superfamily member 19 \\
\hline v1.01640 & K05161 & Tumor necrosis factor receptor superfamily member 26 \\
\hline v1.08207; v1.16237; v1.14824 & K10133 & Tumor protein p53-inducible protein 3 \\
\hline
\end{tabular}

significantly to the protection of coral reef ecosystems against the detrimental effects of organic enrichment $[351,352]$. One emerging threat to coral reefs is the outbreak of infectious diseases (reviewed in [353]). Although highly subjective and with little experimental evidence to date, the coral probiotic hypothesis [354] suggests that the coral prokaryotic microbiome can adapt to changing environmental conditions by selective microbial reorganisation to impart greater resistance to disease and pathogenmediated bleaching [355]. Whether the coral microbiome can respond to changing environmental conditions more rapidly than by host genetic mutation and selection based on contemporary phenotypic evolution on ecological time-scales [356], is a topic of current debate [357].

Corals, like other invertebrates, have an innate immune system based on self-histocompatibility recognition (reviewed in [358]), but to date few adaptive components have been identified [359]. Corals do not produce antibodies and thus lack a true adaptive immune system. Nonetheless, corals once susceptible to infection and bleaching caused by a specific bacterial agent can become immune to the invading pathogen by a phenomenon termed "experience-mediated tolerance", a precept of the hologenome theory of evolution [360], although how this process occurs is largely unknown. In our annotation of the genome sequence of $A$. digitifera we uncovered genes encoding the expression of disease resistance proteins (Table 13), two of which match the plant RPM1 and RPS2 pathogen resistance proteins that guard against disease by binding with pathogen avirulence receptors [360,361]. Significant also is a gene to express the pathogenesis-related protein PR-1 (29 sequence domain matches) that is inducible in plants for systemic acquired resistance to pathogenic invasion [362]. We uncovered also multiple genes encoding the expression of myeloperoxidase (MPO) enzymes. MPOs produce hypochlorous acid from hydrogen peroxide and chloride ion (requiring heme as a cofactor), and it oxidizes tyrosine to the tyrosyl radical using hydrogen peroxide as an oxidizing agent. Hypochlorous acid and tyrosyl radicals are strong cytotoxic agents that in higher organisms are used as a primary defence by neutrophils to protect against invading pathogens. Phenoloxidase (tyrosinase) activity is reported to contribute to the innate defence system of $A$. millepora and Porites sp. 
Table 13 Microbial symbiosis and pathogenicity proteins in the predicted proteome of $A$. digitifera

\begin{tabular}{|c|c|c|}
\hline Gene sequence & KEGG Orthology & Encoded protein description \\
\hline v1.06126 & K13061 & Acyl homoserine lactone synthase \\
\hline v1.19990 & K01372 & Bleomycin hydrolase \\
\hline v1.00209; v1.06178 & K03587 & Cell division protein Ftsl (penicillin-binding protein 3) \\
\hline v1.18860 & K13458 & Disease resistance protein \\
\hline v1.16231; v1.00374; v1.08191 & K13457 & Disease resistance protein RPM1 \\
\hline v1.13482 [+ 4 other sequence copies] & K13459 & Disease resistance protein RPS2 \\
\hline v1.07889 & K12090 & Cag pathogenicity island protein 5 \\
\hline v1.24345 & K12091 & Cag pathogenicity island protein 6 \\
\hline v1.18924; v1.17622 & K12093 & Cag pathogenicity island protein 8 \\
\hline v1.05278 & K12096 & Cag pathogenicity island protein 11 \\
\hline v1.02083 & K12104 & Cag pathogenicity island protein 19 \\
\hline v1.12907 & K12109 & Cag pathogenicity island protein 24 \\
\hline v1.00209; v1.06178 & K03587 & Cell division protein Ftsl (penicillin-binding protein 3) \\
\hline v1.13874 & K07259 & Carboxy/endopeptidase (penicillin-binding protein 4) \\
\hline v1.12514; v1.09758 & K04127 & Isopenicillin-N epimerase \\
\hline v1.21332 & K04126 & Isopenicillin- $N$ synthase \\
\hline v1.07742 & K02547 & Methicillin resistance protein \\
\hline v1.17478; v1.16977; v1.10289; v1.19907 & K04079 & Molecular chaperone HtpG (anti-bacterial) \\
\hline v1.08255 & K13651 & Motility quorum-sensing regulator, GCU-specific toxin \\
\hline v1.14792 [+ 7 other sequence copies] & K10789 & Myeloperoxidase \\
\hline v1.02333 [+ 26 other sequence copies] & K13449 & Pathogenesis-related protein 1 \\
\hline v1.05017 & K03693 & Penicillin-binding protein \\
\hline v1.17507 & K12556 & Penicillin-binding protein $2 X$ \\
\hline v1.13874 & K07259 & Penicillin-binding protein 4 \\
\hline v1.16655 & K02171 & Penicillinase repressor \\
\hline v1.14688 & K15126 & Type III secretion system cytotoxic effector protein \\
\hline v1.20647 & K03980 & Virulence factor, integral membrane protein \\
\hline V1.18964 & K03810 & Virulence factor, oxidoreductase domain \\
\hline
\end{tabular}

[363] via activation of the melanin-signalling pathway that is induced in response to coral bleaching and localised disease $[364,365]$. Three genes of $A$. digitifera encode tyrosinase enzymes (data not tabulated) to account for the phenoloxidase activity reported in corals.

The genome of $A$. digitifera also reveals homologues of genes that promote bacterial pathogenicity (Table 13), including virulence factors that are expressed and excreted by invading pathogens (bacteria, viruses, fungi and protozoa) to inhibit certain protective functions of the host. Such are the bacterial Type III cytotoxic effector protein and multiple Type IV Cag pathogenicity island proteins encoded in the coral genome. Many Gram-negative bacteria utilize Type III secretion proteins, which are regulated by quorum sensing, to deliver cytotoxic effector proteins into eukaryote host cells during infection. Cag (cytotoxin-associated) pathogenicity island (PAI) proteins are encoded by mobile genetic elements of the Type IV system secreting both proteins and large nucleoprotein complexes [366] that may be transferred between prokaryotes to enhance selected traits of virulence [367]. Our annotation reveals genes encoding six pathogenicity island proteins (Table 13) with similarity to the Cag PAI proteins of the human Heliobacter pylori, an infectious bacterium causing peptic ulcers that may lead to the development of stomach cancer. While many properties of Type III and IV secretion system proteins have been well characterized in bacteria, the functional purpose of homologous genes in $A$. digitifera, if expressed, are unknown.

The genome of $A$. digitifera contains genes of bacterial origin that encode the motility quorum-sensing regulator of the GCU-specific mRNA interferase toxin and acyl homoserine lactone synthesis used for the communication 
of quorum sensing between bacteria to enable the coordination of group behaviour based on collective population density. Apparent in our annotation (Table 13) is a wide array of microbial penicillin-binding proteins (PBPs) that have an affinity for $\beta$-lactam antibiotics that by binding to PBPs prevent bacteria from constructing a cell wall. There are genes also to enhance antibiotic resistance, including potential expression of a penicillinase repressor, a methicillin resistance protein and bleomycin hydrolase (cysteine peptidase). Additionally, isopenicillin- $\mathrm{N}$ synthase and an isopenicillin- $\mathrm{N}$ epimerase, both of which catalyse key steps in the biosynthesis of penicillin and cephalosporin antibiotics, are encoded in the coral genome. Taken as a whole, we demonstrate an extensive presence of ancient nonmetazoan genes that are maintained in the genome of $A$. digitifera, as is reported in the genomes of $A$. millepora and the anemone $N$. vectensis [368]. Recent thought on genome evolution places these ancestral conserved domains as 'orphan' or 'taxonomically restricted' genes [352,369,370], rather than acquired later by horizontal gene transfer. There is, of course, little knowledge of how or when, if at all, these non-metazoan genes are expressed or even their function to mediate pathogenicity in the coral holobiont.

\section{Proteins of viral pathogenicity}

Marine viruses were of minor interest until 1989, when it was realised that virus-like particles (VLPs) are the most abundant biological entities to occupy aquatic environments with variable numbers reaching $\sim 10^{8}$ VLPs $\mathrm{ml}^{-1}$ [371]. Typically, VLPs surpass the number of marine bacteria by an order of magnitude in coastal waters [372]; their diversity is extremely high and many are specific to the marine environment [373,374]. Significant VLP numbers are reported from the surrounding waters of oceanic coral reef atolls [375], in waters flowing across the reef substratum [376] and in samples taken within the close vicinity of coral colonies [377,378]. The viral load within the surface microlayer of scleractinian corals is enumerated as being $10^{7}-10^{8}$ VLPs $\mathrm{mL}^{-1}$ [379] and, based on VLP morphological diversity, is attributed to infecting various microbial hosts (bacteria, archaea, cyanobacteria, fungi and algae) residing within the coral mucus [380]. VLPs have been observed in the epidermal and gastrodermal tissues of corals and occasionally occur in the mesogloea [381]. Latent viruses were found to infect Symbiodinium isolated from several scleractinian corals [382-384] with a preponderance of eukaryotic algaeinfecting phycodnaviruses suggested [385]. A wide range of bacteriophage and eukaryotic virus families have been identified within scleractinians using metagenomic analyses [207,386-388], with bacteriophages being by far the most abundant entities (Wood-Charlson EM, Weynberg KD, Suttle CA, Roux S, van Oppen MJH: Methodological biases in coral viromics, submitted).
The importance of the coral-virus interactome in bleaching and disease (reviewed in [185,389]) is founded on reports showing that VLP abundances are higher in the seawater immediately surrounding diseased compared to that of healthy corals [378], that latent viruses are induced by heat stress in symbiotic dinoflagellates of the sea anemone Anemonia virdis [382] and the coral Pavona danai [383], and that UV exposure induces a latent virus-like infection in cultured Symbiodinium [187]. Quantitative 454 pyrosequence analysis of the coral Porites compressa on exposure to reduced $\mathrm{pH}$, elevated nutrients or thermal stress showed that the abundance of its viral consortia varied across treatments, but notably a novel herpes-like virus increased by up to 6 orders of magnitude on exposure to abiotic stress [387], although some caution may be warranted in assessing the reliability of such determinations [Wood-Charlson et al., submitted]. Unexpectedly, the proteome of an endosymbiont-enriched fraction of the coral Stylophora pistillata showed a significant 114-fold increase in a viral replication protein on thermal bleaching [39], which is consistent with the finding of VLP induction in $P$. compressa by similar treatment [387].

General aspects of histocompatibility [390-393] and the genetic structure of innate immune receptors of the Cnidaria [363,394-401], including the immune response effected by coral disease and bleaching [364,402], have been examined extensively, hence further elaboration here is unnecessary. Instead, we focus on proteins that directly regulate the pathogenicity of coral-associated microbes and viruses. The $A$. digitifera genome encodes protein homologues having either putative antiviral and viruspromoting activities (Table 14). These homologues include the antiviral "superkiller" helicase SKI2 protein that acts by blocking viral mRNA translation [403] and, together with the superkiller proteins SKI3 (69 sequence alignments) and SKI8 of the exosome complex, function in a $3{ }^{\prime}$-mRNA degradation pathway [404]. The coral genome encodes also three exoribonuclease (RNase) enzymes (XRN, XRN2 and RNB) with antiviral RNAdegrading properties $[405,406]$. Annotation of the coral genome reveals homologues to four interferon proteins (IFNB, IFNG, IFNW1 and IFNT1). Interferons are potent and selective antiviral cytokines [407], which are induced by viral infection or by sensing dsRNA, a byproduct of viral replication, leading to the transcription of interferon-stimulated genes whose products have antiviral activities and others having antimicrobial, antiproliferative/antitumor or immumomodulatory effects [408,409]. Included in the coral antivirus defence system are three members of the interferon regulatory transcription factor (IRF1, IRF2 and IRF8) family proteins. IRF1 and IRF2 are transcriptional activators of cytokines and other target genes [410]; IRF1 is known to trans-activate the tumor suppressor protein p53 [411] while IRF2 regulates post- 
Table 14 Regulatory and related proteins of viral pathogenicity in the predicted proteome of $A$. digitifera

\begin{tabular}{|c|c|c|}
\hline Gene sequence & KEGG Orthology & Encoded protein description \\
\hline v1.20647; v1.06188; v1.21287 & K12599 & Antiviral helicase SKI2 \\
\hline v1.18443 [+ 40 other sequence copies] & K12807 & Baculoviral IAP repeat-containing protein 1 (BIRC1) \\
\hline v1.06263 [+ 6 other sequence copies] & K04725 & Baculoviral IAP repeat-containing protein 2/3/4 (BIRC2/3/4) \\
\hline v1.14355 & K08731 & Baculoviral IAP repeat-containing protein 5 (BIRC5) \\
\hline v1.04171 [+ 7 other sequence copies] & K10586 & Baculoviral IAP repeat-containing protein 6 (BIRC6) \\
\hline v1.12348; v1.01945; v1.16612 & K06731 & Bone marrow stromal cell antigen 2 (antiviral BST2) \\
\hline v1.01539 [+ 7 other sequence copies] & K04012 & Complement component receptor 2 (CR2) \\
\hline v1.17305 & K04462 & Ecotropic virus integration site 1 protein (EVI1) \\
\hline v1.1496 [+ 4 other sequence copies] & K12618 & 5'-3' Exoribonuclease 1 (antiviral XRN1) \\
\hline v1.22746; v1.19002; v1.12850; v1.21216 & K12619 & 5'-3' Exoribonuclease 2 (antiviral XRN2) \\
\hline v1.09005 & K01147 & Exoribonuclease II (antiviral RNB) \\
\hline v1.22793; v1.12978; v1.19008; v1.20838 & K09239 & HIV virus type I enhancer-binding protein (HIVEP) \\
\hline v1.02776 [+ 7 other sequence copies] & K15046 & Influenza virus NS1A-binding protein (NS1A-BP) \\
\hline v1.09829; v1.13077 & K05415 & Interferon beta (IFNB) \\
\hline v1.11946; v1.21512; v1.11221; v1.11927 & K04687 & Interferon gamma (IFNG) \\
\hline v1.21512 & K14140 & Interferon gamma induced GTPase (ITGP) \\
\hline V1.11946 & K05133 & Interferon gamma receptor 2 (IFNGR2) \\
\hline v1.01539 [+ 4 other sequence copies] & K04012 & Interferon-induced GTP-binding protein Mx1 \\
\hline v1.10782; v1.23797; v1.17119; v1.03221 & K12647 & Interferon-induced helicase $\mathrm{C}$ domain-containing protein 1 \\
\hline v1.06274; v1.15849; v1.05943 & K06566 & Interferon induced transmembrane protein (IFITM1) \\
\hline v1.21327; v1.24081 & K05440 & Interferon, omega 1 (IFNW1) \\
\hline v1.11817 & K09444 & Interferon regulatory factor 1 (IRF1) \\
\hline v1.11816; v1.07639 & K10153 & Interferon regulatory factor 2 (IRF2) \\
\hline v1.11421 & K10155 & Interferon regulatory factor 8 (IRF8) \\
\hline v1.02158 & K12579 & Interferon-stimulated gene 20 kDa protein (ISG20) \\
\hline v1.15947 & K05442 & Interferon tau-1 (IFNT1) \\
\hline v1.22825; v1.08034; v1.08520 & K05788 & Integration host factor subunit beta (IHFB) \\
\hline v1.14899 & K08220 & MFS transporter, FLVCR family virus subgroup C receptor \\
\hline v1.04514; v1.04513; v1.16929 & K12648 & Mitochondrial antiviral-signalling protein (MAVS) \\
\hline v1.17718; v1.08002; v1.08001; v1.22382 & K06081 & Poliovirus receptor-related protein 1 (PVRL1) \\
\hline v1.21413; v1.06637 & K06531 & Poliovirus receptor-related protein 2 (PVRL2) \\
\hline v1.11740; v1.21467; v1.11410; v1.17135 & K06592 & Poliovirus receptor-related protein 3 (PVRL3) \\
\hline v1.15077 & K06593 & Poliovirus receptor-related protein 4 (PVRL4) \\
\hline v1.04158 [+ 68 other sequence copies] & K12600 & Superkiller protein 3 (antiviral SHI3) \\
\hline v1.18238 [+ 4 other sequence copies] & K12601 & Superkiller protein 8 (antiviral SHI8) \\
\hline
\end{tabular}

transcriptional induction of NO synthase [412]. Conversely, IRF8 is an interferon consensus sequencebinding protein that is a negative (interference) regulator of enhancer elements common to interferon-inducible genes [413]. The coral genome additionally includes an interferon-stimulated $20 \mathrm{kDa}$ protein (ISG20) RNase specific to deactivation of singled-stranded RNA viruses [414]. The coral genome encodes several interferoninducible proteins, notably interferon gamma induced
GTPase (IGTP) that accumulates in response to IFNB [415], the interferon-induced GTP-binding protein Mx1 that is a key element of host antiviral defence [416], the interferon-induced helicase $\mathrm{C}$ domain-containing protein1 (aka MDA-5), which is an immune receptor that senses viral dsRNA to activate the interferon antiviralresponse cascade [417] and the interferon-induced transmembrane protein (IFITM1) that suppresses cell growth [418]. The coral genome encodes the interferon-gamma 
receptor 2 (IFNGR2) transmembrane protein that activates downstream signal transduction cascades that control cell proliferation and apoptosis [419]. Encoded also is a homologue of the human bone marrow stromal cell antigen 2 (BST2) that inhibits retrovirus infection by preventing VLP release from infected cells [420]. Additionally encoded is a mitochondrial antiviral-signalling protein (MAVS) that triggers the host immune response by activation of the nuclear transcription factor NF-kB and the interferon regulatory transcription factor IRF3 which coordinates the expression of type-1 interferons such as IFNB [421].

The coral genome encodes a full set of baculoviral IAP repeat-containing proteins BIRC 1-6 (Table 14). The IAP (inhibitor of apoptosis) family proteins were first identified secreted by baculovirus to protect infected cells from death in the progression of viral replication [422]. Expressed by most eukaryotic organisms (reviewed in [423]), their IAP function is presumably conserved in corals. The coral genome encodes a full set of poliovirus receptor-related proteins (PVRL1-4) of the immunoglobulin superfamily, which bind and transport herpesviruses at the cellular membrane in the establishment of latent infections (reviewed in [424]). Encoded also is a complement component (3d/Epstein Barr virus) receptor 2 (CR2) protein that binds to the Epstein-Barr virus Herpes viridae with antigenic activity for disease prevention [425]. Another encoded protein is a homologue of the human immunodeficiency virus type 1 (HIV-1) enhancerbinding protein (HIVEP; aka EBP1) that attaches to the HIV long terminal repeat (LTR) region to activate transcription via the HIV LTR [426]. Present in the coral genome is also a homologue of the influenza virus nonstructural binding protein NS1A-BP that interacts with the NS1 virulence factor of the influenza A virus Orthomyxoviridae to interfere with NS1-inhibition of pre-mRNA splicing within the host nucleosome [427]. NS1A-BP inhibits NS1A-mediated disruption of the host immune response caused by restricting interferon production and the antiviral effects of IFN-induced proteins [428]. The genome of $A$. digitifera encodes an integration host factor subunit beta (IHFB), first discovered as a host factor for bacteriophage $\lambda$ integration of mobile genetic elements, that in $E$. coli is involved in multiple processes of DNA replication, site-specific recombination and gene expression [429]. A homologue of the MFS transporter feline leukemia virus subgroup C receptor (FLVCR) cell surface protein is encoded in the coral genome, which in cats confers susceptibility to FeLV-C infection [430]. Encoded also is a viral integration site 1 (EVI1) that in humans is an oncogenic transcription factor, often activated by viral infection, to cause proliferation of invasive tumours [431]. Arguably, these homologue proteins typically expressed in such distantly related species may have similar relevance in viral interactions of the coral holobiome.

How these regulatory proteins and viral receptors interact and respond to viral infection in corals is yet to be realised. The absence of virion-specific sequences (e.g. for nucleic acid replication or capsid structure) suggests that proviral DNA is absent from the coral genome, or it may be an artefact of the limited number of marine viral sequences deposited in public databases. Discovery of viral activity through proteomics [39] may, therefore, suggest that viral proteins are synthesised from a lytic infection, but this requires confirmation.

\section{Toxins and venom}

A review of protein sequences deposited in the UniProt database in October 2012 shows that there are 150 known cnidarian toxins. These toxins have diverse biological activities (neurotoxins, pore-forming cytolysins and venom phospholipases) used to capture prey and for protection against predators [432] that are best characterised in sea anemones (Actiniaria) with 141 sequences deposited [433,434]. The cytotoxin MCTx-1 isolated from the Net Fire Coral Millepora dichotoma is the only toxin from a coral deposited in Uniprot (accession number A8QZJ5). However, our initial examination of the predicted proteome of $A$. digitifera shows 18 proteins with similarity to bacterial toxins and associated regulatory proteins (Table 15). Unlike reports from proteomic examination of the coral S. pistillata [39] and nematocysts (stinging organelles) of the jellyfish Olindias sambaquiensis [435], Tamoya haplonema, Chiropsalmus quadrumanus, Chrysaora lactea (PF Long et al., pers comm), by sea anemones [434] and by the highly dangerous box jellyfish Chironex fleckeri [436,437], no venoms typical of higher animals were found in the A. digitifera genome. This was because our annotation was carried out using the KEGG database (release v58 [53]) to relate $A$. digitifera protein sequences to KEGG orthologues. The KEGG database is a collection of proteins from well characterised and ubiquitous biochemical pathways. Animal venoms, however, are highly specialised proteins for which this release of the KEGG database does not contain any described orthologues.

KEGG orthology-based annotation of the A. digitifera genome reveals genes encoding protein homologues of 10 bacterial toxins, 7 regulatory toxin proteins and a botulinum protein substrate (Table 15). Of the 9 toxin homologues, one with similarity to anthrax edema factor (EF) adenylate cyclase (CyaA) is one of three proteins that comprise the anthrax toxin of Bacillus anthracis, the other two being a protective antigen (PA) and lethal factor (LF). Without the LF protein, anthrax CyaA has no known toxic effects in animals [438], although the EF protein does play an important role in disabling 
cellular functions vital for microbial host defences [439]. The $A$. digitifera genome encodes a secretion virulence factor exotoxin A-like protein produced by Pseudomonas aeruginosa, which for this bacterium affects local tissue damage, bacterial invasion and immunosuppression within their eukaryote host [440] with pathogenicity similar to that of the diphtheria toxin [441]. Another encoded protein is a murine-like toxin (Ymt) produced by the enterobacterium Yersinia pestis, which is the causative agent responsible for transmission of the notorious bubonic plague [442]. Additionally, two hemolytic enterotoxins similar to NheA and NheBC produced by Bacillus cereus [443], an enterotoxin (EntA) similar to that of Staphylococcus aureus [444], a Shiga-like enterotoxin (StxB) produced by Shigella dysenteria, the diarrhoea-causing toxin A/B (TcdAB) such as that secreted by Clostridium difficile [445], and a protein similar to the zonula occludens (tight junction) enterotoxin (Zot) secreted by Vibrio cholera [446] are encoded in the A. digitifera genome. Within the predicted proteome is also a homologue of the vacuolating cytotoxin (VacA) produced by Helicobacter pylori that colonises the gastric mucosa of the human stomach epithelium [447].

Although a direct homologue of the cholera toxin (CT) was not found encoded in the $A$. digitifera genome (Table 15), a protein similar to its transcriptional activator ToxR was. ToxR not only controls the expression of CT in Vibrio cholera [448], but also a co-regulated pilin (TcpA) protein that is under control of the ToxR regulon cascade [449]. Bacterial TcpA protein is assembled into toxincoregulated pili that induce the transfer of DNA by horizontal exchange of genetic material during conjugation [450]. TcpA and two toxin co-regulated biosynthetic proteins (TcpI and Tcps) of the bacterial virulence-associated pilus appendage [451] are encoded in the coral genome. Entrained also are the motility quorum-sensing interference regulator MsqR and its transcriptional regulator MsqA that in Eschericia coli controls biofilm formation by inhibiting quorum-sensing motility, and together the MqsR/MqsA complex represses the lethal cold shock-like protein cspD gene [452] that on expression impairs DNA replication [453]. The A. digitifera genome likewise encodes a Type III secretion system T3SS cytotoxic effector (BteA) protein [454] that in Gram-negative invasive bacteria is translocated into host cells to suppress innate immunity to enhance virulence $[455,456]$. However, the ecophysiological significance of these toxigenic proteins and allied regulators, if indeed expressed by the coral genome, is unknown.

In addition to using the KEGG database, we undertook a BLAST search of the predicted proteome of $A$. digitifera against peptide sequences for all animal venoms using the annotated UniProtKB/Swiss-Prot Tox-Prot program [457]. This search revealed a large number of accession hits from the predicted proteome, although these are unlikely to be
Table 15 Proteins homologous to bacterial toxins in the predicted proteome of $A$. digitifera

\begin{tabular}{|c|c|c|}
\hline Gene sequence & KEGG Orthology & Encoded protein description \\
\hline v1.20214 & K11029 & $\begin{array}{l}\text { Anthrax edema toxin adenylate } \\
\text { cyclase (CyaA) }\end{array}$ \\
\hline v1.17686 & K10921 & $\begin{array}{l}\text { Cholera toxin transcriptional } \\
\text { activator (ToxR) }\end{array}$ \\
\hline v1.13017 & K11020 & Exotoxin A (ToxA) \\
\hline v1.23507 & K13655 & $\begin{array}{l}\text { HTH-type transcriptional } \\
\text { regulator (MsqA) antitoxin for } \\
\text { MqsR }\end{array}$ \\
\hline$\vee 1.21184$ & K11009 & Murine toxin (Ymt) \\
\hline v1.04313 & K11033 & $\begin{array}{l}\text { Non-hemolytic enterotoxin A } \\
\text { (NheA) }\end{array}$ \\
\hline v1.08011 & K11034 & $\begin{array}{l}\text { Non-hemolytic enterotoxin } B / C \\
\text { (NheBC) }\end{array}$ \\
\hline v1.08255 & K13651 & $\begin{array}{l}\text { Motility quorum-sensing } \\
\text { regulator (MqsR) interferase } \\
\text { toxin }\end{array}$ \\
\hline V1.15986 & K11059 & Probable enterotoxin A (EntA) \\
\hline v1.13046 & K04392 & $\begin{array}{l}\text { Ras-related C3 botulinum toxin } \\
\text { substrate } 1 \text { ( } \operatorname{Rac} 1 \text { ) }\end{array}$ \\
\hline V1.13966 & K11007 & Shiga toxin subunit B (StxB) \\
\hline v1.23958 & K11063 & Toxin A/B (TcdAB) \\
\hline v1.21174 & K10930 & Toxin co-regulated pilin (TCP) \\
\hline v1.05802 & K10961 & $\begin{array}{l}\text { Toxin co-regulated pilus } \\
\text { biosynthesis protein I (Tcpl) }\end{array}$ \\
\hline v1.21783 & K10964 & $\begin{array}{l}\text { Toxin co-regulated pilus } \\
\text { biosynthesis protein } \mathrm{S}(\mathrm{TcpS})\end{array}$ \\
\hline v1.14688 & K15126 & $\begin{array}{l}\text { Type III secretion system } \\
\text { cytotoxic effector protein } \\
\text { (BteA) }\end{array}$ \\
\hline v1.05520 & K11028 & Vacuolating cytotoxin (VacA) \\
\hline v1.06590 & K10954 & Zona occludens toxin (Zot) \\
\hline
\end{tabular}

true multiple copies given that the genome sequence has yet to be completely assembled. However, just taking a single accession number from each annotation reveals a complex array of 83 toxins that represents the predicted venom of $A$. digitifera (Table 16); UniProt BLAST E-values are given in Additional file 1: Table S16b. These venoms are highly diverse and are significantly homologous to toxins from a wide variety of venomous marine and terrestrial creatures such as fish, reptiles, other cnidarians, conesnails, stinging insects and even a venomous mammal (Shrew), covering the complete range of pharmacological properties known in venoms, including cytolytic, neurotoxic, haemotoxic, phospholipase, proteinase and proteinase inhibitor activities. Both the number of toxins predicted in the venom of $A$. digitifera and the degree of homology to such widely divergent phyla is remarkable. Accordingly, cnidarian venoms may possess unique biological properties that might generate new 
Table 16 UniProt homologues of animal venom proteins in the predicted proteome of $A$. digitifera

\begin{tabular}{|c|c|c|}
\hline Gene sequence & UniProt toxin accession & Animal with closest homology \\
\hline $\begin{array}{r}\text { v1.01916 [+ } 5 \text { other sequence } \\
\text { copies }]\end{array}$ & Q92035; Acetylcholinesterase & Bungarus fasciatus (Banded Krait) \\
\hline $\begin{array}{r}\text { v1.06761; v1.08075; v1.09840; } \\
\text { v1.20323 }\end{array}$ & Q91AM1; Agkisacutacin (subunit anticoagulant protease) & Deinagkistrodon acutus (Sharp-nosed Viper) \\
\hline v1.04809 & A8QL52; L-Amino acid oxidase & Bungarus fasciatus (Banded Krait) \\
\hline v1.06380 & Q4JHE1; L-Amino acid oxidase & Pseudechis australis (Mulga Snake) \\
\hline v1.10291 & P81383; L-Amino acid oxidase & Ophiophagus hannah (King Cobra) \\
\hline v1.14412 & A6MFL0; L-Amino acid oxidase & Demansia vestigiata (Lesser Black Whipsnake) \\
\hline v1.16469 & P81383; L-Amino acid oxidase & Ophiophagus hannah (King Cobra) \\
\hline v1.23477 & P81382; L-Amino acid oxidase & Calloselasma rhodostoma (Malayan Pit Viper) \\
\hline v1.16440 & C5NSL2; Bandaporin (haemolysin) & Anthopleura asiatica (Sea Anemone) \\
\hline $\begin{array}{r}\text { v1.16571 [+ } 10 \text { other sequence } \\
\text { copies }]\end{array}$ & Q76B45 ; Blarina toxin (vasoactive protease) & Blarina brevicauda (Northern Short-Tailed Shrew) \\
\hline $\begin{array}{r}\text { v1.06055 [+ } 20 \text { other sequence } \\
\text { copies }]\end{array}$ & Q593B6; Coagulation factor V & Pseudonaja textilis (Eastern Brown Snake) \\
\hline v1.07831; v1.10094; v1.20732 & P14530; Coagulation factor IX & Protobothrops flavoviridis (Okinawa Habu Snake) \\
\hline $\begin{array}{r}\text { v1.01708 [+ } 5 \text { other sequence } \\
\text { copies }]\end{array}$ & Q4QXT9; Coagulation factor X & Tropidechis carinatus (Rough-Scaled Snake) \\
\hline v1.09601; v1.10410 & Q93109; Equinatoxin-5 (cytolysin) & Actinia equina (Beadlet Anemone) \\
\hline v1.06821 & Q08169; Hyaluronidase & Apis mellifera (European Honey Bee) \\
\hline v1.08924 & IOCME7; Hyaluronidase, Conohyal-Cn1 & Conus consors (Singed Cone) \\
\hline $\begin{array}{r}\text { v1.06189 [+ } 112 \text { other sequence } \\
\text { copies] }\end{array}$ & Q9XZC0; a-Latrocrustotoxin Lt1a (neurotoxin) & $\begin{array}{l}\text { Latrodectus tredecimguttatus (Mediterranean Black } \\
\text { Widow Spider) }\end{array}$ \\
\hline $\begin{array}{r}\text { v1.02942 [+ } 8 \text { other sequence } \\
\text { copies] }\end{array}$ & GOLXV8; a-Latrocrustotoxin Lh1a (neurotoxin) & Latrodectus hasseltii (Australian Redback Spider) \\
\hline $\begin{array}{r}\text { v1.00644 [+ } 32 \text { other sequence } \\
\text { copies] }\end{array}$ & Q25338; A- Latroinsectotoxin Lt1a (neurotoxin) & $\begin{array}{l}\text { Latrodectus tredecimguttatus (Mediterranean Black } \\
\text { Widow Spider) }\end{array}$ \\
\hline v1.07446 & A7X3X3; Lectin, Lectoxin Enh4 (platelet binding) & Enhydris polylepis (Macleay's Water Snake) \\
\hline v1.20653 & A7X3Y6; Lectin, Lectoxin Enh7 (platelet binding) & Enhydris polylepis (Macleay's Water Snake) \\
\hline v1.02561,v1.11493; v1.16681 & A7X3Z4; Lectin, Lectoxin Lio1 (platelet binding) & Liophis poecilogyrus (Water Snake) \\
\hline $\begin{array}{r}\text { v1.13597; v1.08696; v1.10757; } \\
\text { v1.20654 }\end{array}$ & A7X3Z7; Lectin, Lectoxin Lio2 (platelet binding) & Liophis poecilogyrus (Water Snake) \\
\hline v1.18386, v1.15479 & A7X413; Lectin, Lectoxin Lio3 (platelet binding) & Liophis poecilogyrus (Water Snake) \\
\hline v1.06094 & A7X406; Lectin, Lectoxin Phi1 (platelet binding) & Philodryas olfersii (Green Cobra) \\
\hline v1.06416; v1.16248; v1.23712 & A7X3Z0; Lectin, Lectoxin Thr1 (platelet binding) & Thrasops jacksonii (Black Tree Snake) \\
\hline v1.17681 & Q6TPG9; Lectin, Mucrocetin (platelet binding) & $\begin{array}{l}\text { Protobothrops mucrosquamatus (Brown Spotted Pit } \\
\text { Viper) }\end{array}$ \\
\hline $\begin{array}{r}\text { v1.00077 [+ } 14 \text { other sequence } \\
\text { copies }]\end{array}$ & Q66503; Lectin, Nattectin (platelet binding) & Thalassophryne nattereri (Toad Fish) \\
\hline v1.12241; v1.02332; v1.12298 & Q71RQ1; Lectin, Stejaggregin-A (platelet binding) & Trimeresurus stejnegeri (Bamboo Viper) \\
\hline $\begin{array}{r}\text { v1.02245 [+ } 19 \text { other sequence } \\
\text { copies }]\end{array}$ & A0FKN6; Metalloprotease, Astacin-like toxin & Loxosceles intermedia (Recluse Spider) \\
\hline v1.03638; v1.14772 & Q90391; Metalloprotease, Atrolysin & Crotalus atrox (Western Diamondback Rattlesnake) \\
\hline v1.13106 & D3TTC2; Metalloproteinase, Atragin & Naja atra (Chinese Cobra) \\
\hline v1.11132 & Q7T1T4; Metalloproteinase, BjussuMP-2 & Bothrops jararacussu (Jararacussu Pit Viper) \\
\hline v1.02168 & O73795; Metalloproteinase, Disintegrin & Gloydius brevicaudus (Chinese Mamushi Snake) \\
\hline v1.06910 & Q7SZE0; Metalloproteinase, Disintegrin & Gloydius saxatilis (Rock Mamushi Snake) \\
\hline v1.22282 & P14530; Metalloproteinase, Disintegrin & Protobothrops flavoviridis (Okinawa Habu Snake) \\
\hline
\end{tabular}




\section{Table 16 UniProt homologues of animal venom proteins in the predicted proteome of $\boldsymbol{A}$. digitifera (Continued)}

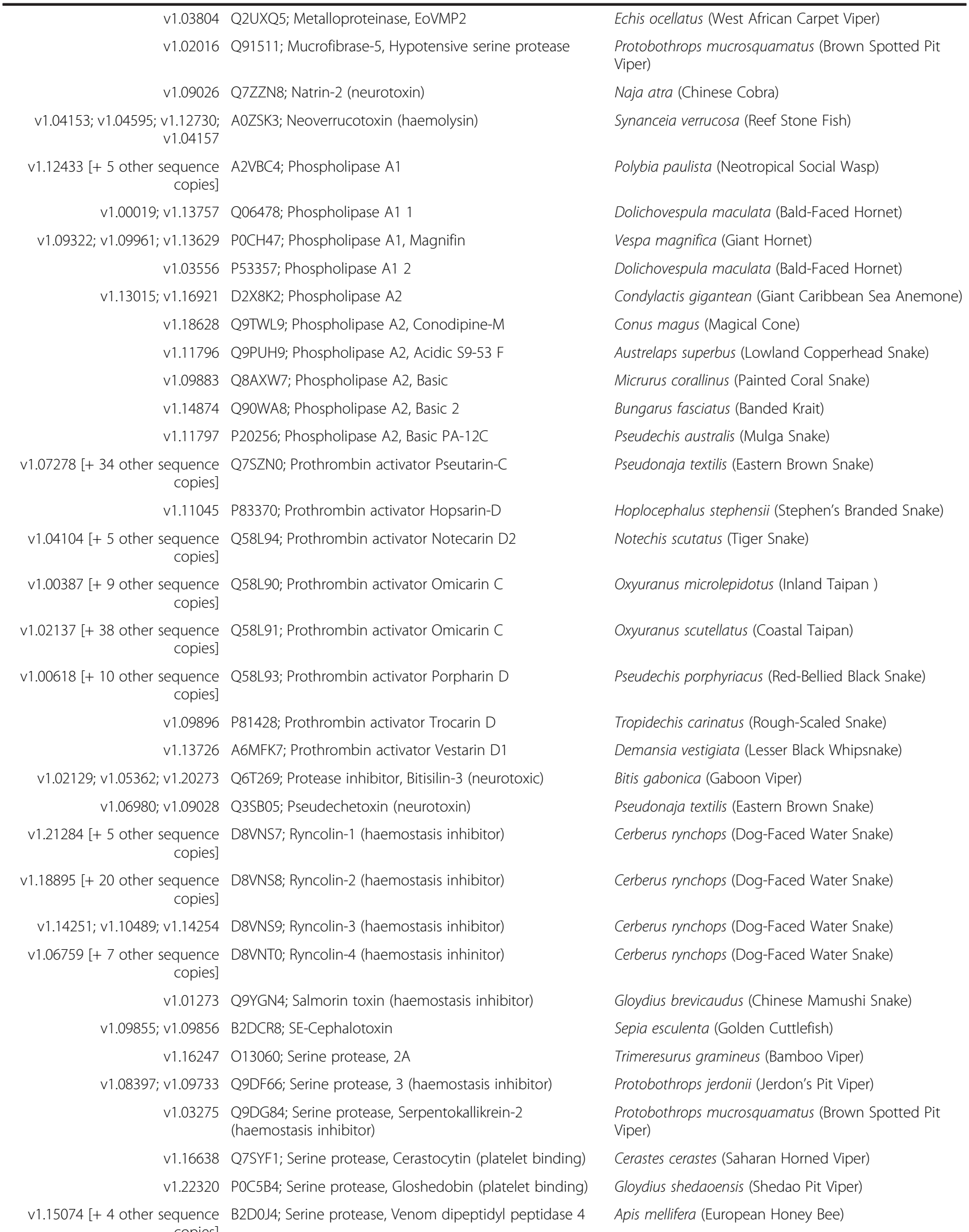




\section{Table 16 UniProt homologues of animal venom proteins in the predicted proteome of $\boldsymbol{A}$. digitifera (Continued)}

\begin{tabular}{|c|c|c|}
\hline v1.05361 & B6RLX2; Serine protease inhibitor, $\mathrm{TCl}$ (neurotoxin) & Ophiophagus hannah (King Cobra) \\
\hline v1.10994 & B7S4N9; Serine protease inhibitor, Taicatoxin (neurotoxin) & Oxyuranus scutellatus (Coastal Taipan) \\
\hline v1.11218; v1.23374 & $\begin{array}{l}\text { Q90WA0; Serine protease inhibitor, Textilinin-2 (thrombin } \\
\text { inhibitor) }\end{array}$ & Pseudonaja textilis (Eastern Brown Snake) \\
\hline v1.17856; v1.22256 & $\begin{array}{l}\text { Q8T3S7; Serine protease inhibitor, U1-aranetoxin-Av1a } \\
\text { (neurotoxin) }\end{array}$ & Araneus ventricosus (Devil Spider) \\
\hline v1.09427; v1.16619; v1.19446 & Q76DT2; Toxin AvTX-60A (cytolysin) & Actineria villosa (Okinawan Sea Anemone) \\
\hline v1.12311 & Q9GV72; Toxin CrTX-A (haemolysin) & Carybdea rastonii (Jimble Jellyfish) \\
\hline $\begin{array}{r}\text { v1.07546 [+ } 5 \text { other sequence } \\
\text { copies }]\end{array}$ & P58911; Toxin PsTX-60 (haemolysin) & Phyllodiscus semoni (Night Anemone) \\
\hline v1.11270; v1.14265 & E2IYB3; Veficolin-1 (complement activator) & Varanus komodoensis (Komodo Dragon) \\
\hline v1.02115 & Q98993; Verrucotoxin (cytolysin) & Synanceia verrucosa (Reef Stonefish) \\
\hline
\end{tabular}

leads in the discovery of novel pharmacologically active drugs. Gene duplication followed by mutation and natural selection is widely held as the key mechanism whereby the large diversity of toxins found within a single venom could have evolved $[458,459]$. Conversely, primary mRNA splicing patterns have been shown to account for the diversity of metalloproteinases in the pit viper Bothrops neuwiedi [460]. Variations in peptide processing have also been shown by proteomics and transcriptomics to explain how a limited set of gene transcripts could generate thousands of toxins in a single species of cone snail [461]. Despite these various processes that could account for the evolution of toxin diversity, it has never been demonstrated how gene duplications or variations in transcript or peptide processing could have radiated across the very different poisonous creatures found on Earth. Our data (Table 16) reveal that the predicted toxins of $A$. digitifera venom are orthologues to all of the most important superfamilies of peptide/protein venoms found in diverse taxa. We posit that the origins of toxins in the venoms of higher organisms may have arisen from deep eumetazoan innovations and that the molecular evolution of these venom super gene families can now be addressed taking an integrated venomics approach using Cnidaria such as the jellyfish as model systems [462].

\section{Detoxification proteins of the chemical defensome}

There have been considerable advancements made to better understand the effects of pollution on coral reef habitats. The three main categories of environmental pollutants from anthropogenic sources are nutrient enrichment (eutrophication), hydrocarbon pollution and heavy metal contamination. Eutrophication from terrestrial inputs are a significant threat to coral reefs stemming from the discharge of treated sewage, the runoff of agricultural fertilizers (plus herbicides and pesticides), and by sedimentation caused by the erosion of organic-rich soils [463]. Notwithstanding that eutrophication can shift coral reef communities towards macroalgae domination [19], nitrogen and phosphorus enrichment can diminish coral growth and affect the photosynthetic performance of their algal symbionts [464]. Nutrient enhancement alters multiple pathways of primary metabolism that in coral is complicated by the photosynthetic demands of its symbiotic partners. While corals respond to hypertrophic levels of nutrients by activating general stress-response proteins [465], there are no specific proteins known to mitigate the cellular effects of nutrient enrichment on corals per se, and we have not attempted to identify such in this study.

Gene families and their regulators that defend against chemical stressors comprise the chemical defensome encoding a network of detoxifying proteins that allows an organism to sense, transform and eliminate potentially toxic endogenous metabolites and xenobiotic contaminants [466]. Expressed proteins of the chemical defensome include the biotransformation cytochrome P450 (CYP) family of enzymes, conjugating enzymes, efflux transporters, heavy metal membrane pump exporters and their transcriptional activators. Annotation of the genome of $A$. digitifera reveals multiple genes encoding 20 hemoproteins belonging to the Phase II cytochrome P450 superfamily of monooxidase enzymes that catalyse the oxidation of diverse organic substances (Table 17). The substrates of CYP enzymes include intermediates of lipid metabolism and sterol/steroid biosynthesis, and include the detoxification of exogenous xenobiotics. Of significance are the CYP1A-type (aryl hydrocarbon hydroxylase) enzymes that have been studied widely in the hepatic response of fishes to polycyclic aromatic hydrocarbon (PAH) contamination (from crude or fuel oil) and exposure to polychlorinated biphenyl and 
Table 17 Proteins of the chemical defensome in the predicted proteome of $A$. digitifera

\begin{tabular}{|c|c|c|}
\hline Gene sequence & KEGG Orthology & Encoded protein description \\
\hline v1.06127; v1.06128 & K01015 & Alcohol sulfotransferase \\
\hline v1.09267 & K00537 & Arsenate reductase \\
\hline v1.24496; v1.24495; v1.03953 & K03893 & Arsenical pump membrane protein \\
\hline v1.10691 & K07755 & Arsenite methyltransferase \\
\hline v1.20443 & K11811 & Arsenical resistance protein ArsH \\
\hline v1.14972 & K01551 & Arsenite-transporting ATPase \\
\hline v1.17644; v1.00480; v1.08150; v1.22865 & K01014 & Aryl sulfotransferase \\
\hline v1.21535; v1.11835; v1.02456 & K01534 & $\mathrm{Cd}^{2+/} \mathrm{Zn}^{2+}$-exporting ATPase \\
\hline v1.03485; v1.21926; v1.05686 & K01533 & $\mathrm{Cu}^{2+}$-exporting ATPase \\
\hline v1.22646 [+ 8 other sequence copies] & K07408 & Cytochrome P450, family 1, subfamily A, polypeptide 1 \\
\hline V1.01284 & K07421 & Cytochrome P450, family 2, subfamily $T$ \\
\hline v1.10544; v1.02314, v1.17490 & K07422 & Cytochrome P450, family 2, subfamily $U$ \\
\hline v1.23039 [+ 13 other sequence copies] & K07422 & Cytochrome P450, family 3 , subfamily A \\
\hline v1.07750 & K07425 & Cytochrome P450, family 4, subfamily A \\
\hline v1.22798; v1.23000 & K07426 & Cytochrome P450, family 4, subfamily B \\
\hline v1.02020 [+ 4 other sequence copies] & K07427 & Cytochrome P450, family 4, subfamily V \\
\hline v1.19495 & K07428 & Cytochrome P450, family 4, subfamily $X$ \\
\hline v1.15382 & K15002 & Cytochrome P450, family 6 \\
\hline v1.16427 & K07430 & Cytochrome P450, family 7, subfamily B \\
\hline v1.17631 & K00498 & Cytochrome P450, family 11 , subfamily A \\
\hline v1.08074 [+ 4 other sequence copies] & K15004 & Cytochrome P450, family 12 \\
\hline v1.02478 [+ 5 other sequence copies] & K00512 & Cytochrome P450, family 17 , subfamily A \\
\hline v1.06713 & K07435 & Cytochrome P450, family 20 , subfamily A \\
\hline v1.22414 [+ 5 other sequence copies] & K07436 & Cytochrome P450, family 24 , subfamily A \\
\hline v1.20153 & K12665 & Cytochrome P450, family 26 , subfamily C \\
\hline v1.08074 [+ 6 other sequence copies] & K00488 & Cytochrome P450, family 27 , subfamily A \\
\hline v1.06537 & K07439 & Cytochrome P450, family 39 , subfamily A \\
\hline v1.22302 [+ 5 other sequence copies] & K07440 & Cytochrome P450, family 46 , subfamily A \\
\hline V1.16335 & K09832 & Cytochrome P450, family 710 , subfamily A \\
\hline v1.18439; v1.02594; v1.02593 & K01016 & Estrone sulfotransferase \\
\hline v1.07758 [+ 5 other sequence copies] & K00699 & Glucuronosyltransferase \\
\hline v1.00764 & K13299 & Glutathione S-transferase kappa 1 \\
\hline v1.17188 & K00799 & Glutathione S-transferase \\
\hline v1.04140 & K07239 & Heavy-metal exporter, HME family \\
\hline v1.10181 & K00481 & p-Hydroxybenzoate 3-monooxygenase \\
\hline v1.16748; v1.07471 & K08365 & MerR family transcriptional regulator, mercuric resistance \\
\hline v1.04382; v1.24424 & K13638 & MerR family transcriptional regulator, Zn(II)-responsive \\
\hline v1.12760 & K08363 & Mercuric ion transport protein \\
\hline v1.04179; v1.01891; v1.00145 & K03284 & Metal ion transporter, MIT family \\
\hline v1.21500 [+ 5 other sequence copies] & K01253 & Microsomal epoxide hydrolase \\
\hline v1.08005 & K08970 & Nickel/cobalt exporter \\
\hline v1.03484 & K08364 & Periplasmic mercuric ion binding protein \\
\hline v1.05406 & K07245 & Putative copper resistance protein $\mathrm{D}$ \\
\hline v1.14635 & K08726 & Soluble epoxide hydrolase \\
\hline v1.01929; v1.19296 & K05794 & Tellurite resistance protein TerC \\
\hline v1.10880; v1.15709; v1.12348 & K07803 & Zinc resistance-associated protein \\
\hline
\end{tabular}


dibenzodioxin toxicants (reviewed in [467]). CYP450 activity has been detected in the corals Favia fragum [468], Siderastrea siderea [469], Montastraea faveolata [470] and Pocillopora damicornis, [471]. Furthermore, CYP encoding sequences have been extracted from the genome of $N$. vectensis [472] and the transcriptome of A. millepora [29]. As well as providing chemical defence, mixed-function CYPs perform multiple endogenous tasks that are often taxon-specific. Hence, the orthology and substrate specificity of coral CYP enzymes cannot be predicted solely on homology to CYPs of known function assigned to higher metazoans. Similar to the function of CPY enzymes, there are genes encoding p-hydroxybenzoate 3-monooxygenase, an oxidoreductase catalyzing aryl oxidation and the soluble and microsomal forms of epoxide hydrolase that converts epoxides, formed by the degradation of aromatic compounds, to trans-diols that by conjugation are readily excreted. Conjugating enzymes to eliminate hydroxylated substrates are the detoxifying UDP-glucuronosyltransferase and sulfotransferase families of enzymes. Estrone sulfotransferase is significant for inactivation of exogenous (contraceptive) estrogens [473] and similar endocrinedisruptive contaminants released from treated wastewater [474]; their occurrence in marine waters are known to disrupt the reproduction and development of fish [475] and corals [476]. Glutathione S-transferase (GST) enzymes catalyse the addition of reduced glutathione to the reactive sites of electrophilic toxins [477]. Surprisingly, only two isoforms of GST were detected in the A. digitifera genome (Table 17), whereas 18 distinct GST-encoding genes (6 classes +1 fungal-type) were classified from genome sequences of $N$. vectensis [472]. This unexpected genome reduction of GST elaboration in A. digitifera begs further examination.

Many toxicological studies on the effects of pollution on cnidarian fitness have focused on their response to heavy metal contamination, including copper, cadmium, mercury and zinc $[478,479]$. In scleractinian corals the uptake and toxic effects of copper [480-483], cadmium [482] and mercury $[484,485]$ have been studied at the metabolic level with specific studies to examine the effects of heavy metal toxicity on coral fertilisation [486-488], settlement [487], metamorphosis [486] and in coral bleaching [489]. Yet, the identification of molecular markers to monitor the response of Cnidaria to sub-lethal levels of heavy metal exposure has been elusive [490]. We were delighted to uncover in our annotation a wide range of genes to express metal-specific (arsenic, copper, mercury, nickel/cobalt and tellurium) resistance, transportation and membrane pump exporting proteins that, together with non-specific heavy metal ion export proteins (Table 17), might prove useful for monitoring the environmental response of $A$. digitifera to heavy metal contamination. Included in the heavy metal defensome are the Mer-family of transcriptional regulators of $\mathrm{Hg}$ - and Zn-resistance proteins and a periplasmic ion- binding protein attributed to the $\mathrm{Hg}$ detoxification system of bacteria [491]. Enzymes specific for arsenic detoxification are an arsenate oxidoreductase for conversion of arsenate to arsenite [492] and arsenite methyltransferase for conversion of arsenite to the less toxic dimethylarsenite that is amenable to excretion [493]. Such processes may enhance the resilience of corals exposed to natural [494] and site-affected [495] levels of arsenic contamination. In contrast, there were no (organo)cyanide detoxification genes apparent in the $A$. digitifera genome, but one sequence (v1.01601; K10814) encodes for hydrogen cyanide synthase of unknown metabolic purpose (data not tabulated). Ancillary evidence suggests that the expression of $\mathrm{HCN}$ synthase could be linked to quorum sensing [496] for regulating microbial densities of the coral holobiont community.

\section{Epigenetic and DNA-remodelling proteins}

In all Kingdoms of life, DNA methylation and chromatin remodelling is pivotal to the regulation of gene transcription independent of underlying allelic variation. One such process mediated by epigenetic changes in eukaryotic biology is the all-important cellular differentiation during morphogenetic development. Epigenetic modifications cause the activation, regulation or silencing of certain genes without changing the basic DNA code. Changes in epigenetic regulation can persist during cell division and across multiple generations [497]. In addition, cytosine methylation may be associated with a higher mutation rate, because deamination of the methylated base produces thymine resulting in $\mathrm{C} / \mathrm{T}$ mutations, which on reproduction may be transmitted by the germline to subsequent generations in selective processes of evolution [498]. On the other hand, environmentally induced destabilisation of the epigenome can produce gene variants (epialleles) that activate transcription and mobilization of DNA transposable elements, which may subsequently lead to stable heritable traits of environmental adaptation, as does occur by genetic imprinting in plants [499]. Transposition has thus the potential to direct increased frequencies of permanent genetic mutations for selective adaptation.

One way by which genes are regulated at the epigenome is through the remodelling of the chromatin histone-DNA complex (the nucleosome), which by post-translational modification changes the template structure of DNA associated histone proteins. These modifications are affected by histone-lysine (and histone-arginine) $\mathrm{N}$-methyltransferase enzymes (Table 18) by which these proteins may be further modified by acetylation, ADP-ribosylation, ubiquination, and phosphorylation (annotation not tabulated). The methylation pattern of histone lysine residues is highly predictive of the gene expression states of transcriptional activation and repression [500]. Necessary epigenomic 
reprogramming of histone modification at different stages of cell development is affected by the activation of histone and lysine-specific demethylase enzymes (Table 18). Determinants for recognition of the histone code are being revealed by a growing body of experimental data providing valuable information on the molecular tractability of binding sites involved in epigenetic signalling [501], which will enhance further insight to epigenetic function.
Direct epigenetic modification of DNA (or mRNA) occurs by methylation of cytosine, and to a lesser extent adenosine and guanine, by nucleobase-specific DNA methyltranferases (Table 18) to give 5-methylcytosine (5$\mathrm{meC}), 3$-methyladenosine (3-meA) and 3-methylguanine (3-meG) nucleotides, respectively. The principal modification product, 5-methylcytosine behaves much like regular cytosine by pairing with guanine, but in areas of high

Table 18 Epigenetic and DNA-remodelling proteins in the predicted proteome of $A$. digitifera

\begin{tabular}{|c|c|c|}
\hline Gene sequence & KEGG Orthology & Encoded protein description \\
\hline v1.04426; v1.02042 & K02528 & 16S rRNA (adenine1518-N6/1519-N6)-dimethyltransferase \\
\hline v1.22358; v1.00249 & K14191 & $18 \mathrm{~S}$ rRNA (adenine1779-N6/1780-N6)-dimethyltransferase \\
\hline v1.19400; v1.04238 & K00561 & 23 rRNA (adenine2085-N6)-dimethyltransferase \\
\hline v1.05107; v1.05242 & K01488 & Adenosine deaminase \\
\hline v1.04152; v1.09790 & K14857 & AdoMet-dependent rRNA methyltransferase SPB1 \\
\hline v1.00197 & K13530 & AraC family transcriptional regulator DNA methyltransferase \\
\hline v1.12967; v1.19789; v1.07763 & K14589 & Cap-specific mRNA (nucleoside-2'-O-)-methyltransferase 1 \\
\hline v1.24281 & K01489 & Cytidine deaminase \\
\hline v1.16211; v1.14952; v1.01094; v1.06983 & K00558 & DNA (cytosine-5-)-methyltransferase \\
\hline v1.19683; v1.05688; v1.04223 & K11324 & DNA methyltransferase 1-associated protein 1 \\
\hline v1.14033; v1.19860; v1.19081; v1.04188 & K11420 & Euchromatic histone-lysine N-methyltransferase \\
\hline v1.02068 & K01487 & Guanine deaminase \\
\hline v1.02920 & K05931 & Histone-arginine methyltransferase CARM1 \\
\hline v1.17589 [+ 7 other sequence copies] & K11446 & Histone demethylase JARID1 \\
\hline v1.07640 & K06101 & Histone-lysine N-methyltransferase ASH1L \\
\hline v1.13515; v1.18577; v1.20187; v1.19182 & K09186 & Histone-lysine N-methyltransferase MLL1 \\
\hline v1.08381 & K09187 & Histone-lysine N-methyltransferase MLL2 \\
\hline v1.24258; v1.19182 & K09188 & Histone-lysine N-methyltransferase MLL3 \\
\hline v1.07992; v1.10302; v1.13829 & K09189 & Histone-lysine N-methyltransferase MLL5 \\
\hline v1.06939; v1.15255; v1.15254 & K11424 & Histone-lysine N-methyltransferase NSD1/2 \\
\hline v1.05552 & K11422 & Histone-lysine N-methyltransferase SETD1 \\
\hline v1.07744 & K11423 & Histone-lysine N-methyltransferase SETD2 \\
\hline v1.03190 & K11431 & Histone-lysine N-methyltransferase SETD7 \\
\hline v1.21867 & K11428 & Histone-lysine N-methyltransferase SETD8 \\
\hline v1.18700 [+ 8 other sequence copies] & K11421 & Histone-lysine N-methyltransferase SETDB \\
\hline v1.07557; v1.11409 & K11419 & Histone-lysine N-methyltransferase SUV39H \\
\hline v1.24733; v1.13497 & K11429 & Histone-lysine N-methyltransferase SUV42OH \\
\hline v1.15405; v1.10291; v1.17601; v1.02845; v1.08629 & K11450 & Lysine-specific histone demethylase 1 \\
\hline v1.23155; v1.09394; v1.17624; v1.05370 & K14835 & Ribosomal RNA methyltransferase Nop2 \\
\hline v1.18460 [+ 6 other sequence copies] & K03500 & Ribosomal RNA small subunit methyltransferase B \\
\hline v1.07407; v1.03110 & K08316 & Ribosomal RNA small subunit methyltransferase D \\
\hline v1.12193 & K02427 & Ribosomal RNA large subunit methyltransferase E \\
\hline v1.11499 & K11392 & Ribosomal RNA small subunit methyltransferase F \\
\hline v1.16053; v1.12676 & K03437 & RNA methyltransferase, TrmH family \\
\hline v1.12453; v1.05459 & K13097 & Methylcytosine dioxygenase \\
\hline
\end{tabular}




\section{Table 18 Epigenetic and DNA-remodelling proteins in the predicted proteome of $A$. digitifera (Continued)}

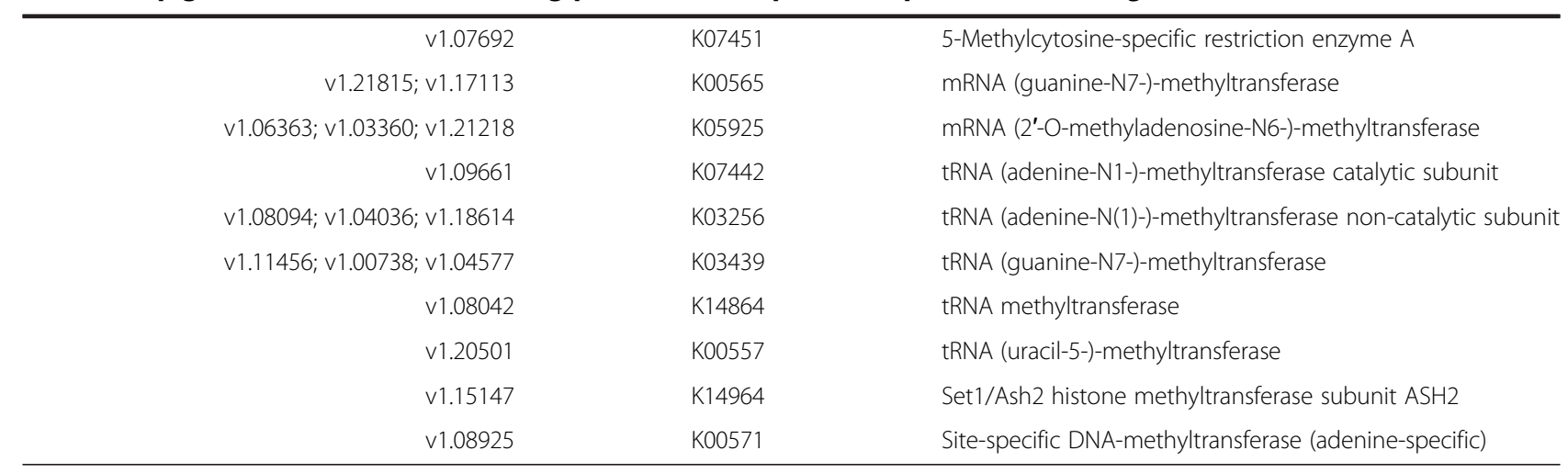

cytosine methylation, genome transcription is strongly repressed (reviewed in [502]), together with the repression of other chromatin-dependent processes, including the incorporation of transposable elements [503]. Alteration in the methylation status of the entire genome, individual chromosomes or at specific gene sites is essential for normal cellular function, but processes for reprogramming methylated DNA at different stages of cell development, unlike the reversal of histone modifications, is poorly defined [504]. While there are abundant enzymes to repair DNA damage caused by spurious $\mathrm{N}$-alkylation, direct nucleotide C-demethylation (via the hypothetical "DNA demethylase" [505]) is thermodynamically infeasible. Instead, removal of epigenetic C-methylated nucleobases occurs by several base-repair pathways involving DNA excision or mismatch repair enzymes. The genome of $A$. digitifera encodes expression of a specific DNA glycosylase enzyme [506] for excision of 3-meA, but there are no such enzymes encoded for the excision of 5-meC and 3-meG, although there is encoded a 5-methylcytosine-specific restriction enzyme. Another pathway for DNA demethylation requires base-specific deamination by the AID/ Apobec family of deaminase enzymes that, for example, converts 5 - $\mathrm{meC}$ to thymine that is replaced subsequently by cytosine by $\mathrm{C} / \mathrm{T}$ mismatch repair enzymes. These methylated nucleobases are recognized for deamination by the cytosine, adenosine and guanine deaminase enzymes [507] that are encoded in the $A$. digitifera genome, and their deaminated bases are subsequently removed by DNA mismatch repair enzymes. Additionally, the genome of $A$. digitifera encodes a methlycytosine dioxygenase enzyme that converts 5-methylcytosine to 5-hydroxymethycytosine (5-hmC), which is recognized for removal by the base excision repair pathway [508] or via its 5-hmC deaminated intermediate [507]. Combined, these DNA demethylation pathways are able to remodel epigenetic modifications at different stages of cell development.

Most current knowledge of DNA and protein methylation comes from studies of mammals and plants, while our understanding of the extent and roles of DNA methylation in invertebrates, marine invertebrates in particular, is still limited [509]. Little is known about the epigenetic potential of corals to acclimatize and adapt to the thermal and synergistic stressors that cause wide-spread coral "bleaching" [510]. Yet, given that acclimatization occurs via the generation of epiallele variants that can in some instances lead to stable heritable traits of environmental adaptation, there is growing interest in the prospect that epigenetic modifications in corals or their algal symbionts [511] may drive adaptation to defend against the damaging threat imposed by rising temperatures from global climate change. It is anticipated that this field of study will rapidly accelerate with the need to better understand epigenetic processes that may contribute to the persistence of coral reefs.

\section{Conclusions}

We offer ZoophyteBase as an unprecedented foundation to interrogate the molecular structure of the predicted $A$. digitifera proteome. Some key findings include proteins with relevance to host-symbiont function, dysfunction and recovery including those that direct vacuolar trafficking and proteins linking symbiont photosynthesis to coral calcification. An extensive catalogue of mammalian-like proteins essential to neural function and venoms related to distant animal phyla suggests their origins lie deep in early eumetazoan evolution. Homologues of prokaryotic genes that have not been described previously in any eukaryote genome such as flagella proteins, proteins essential for nitrogen fixation and photosynthesis point towards lateral gene transfer, perhaps mediated by viruses, that may lead to "shared" metabolic adaptations of symbiosis, and provide corals with limited ability for gene-encoded adaptation to a changing global environment. It is anticipated that understanding how the genome of a coral host interacts with that of its vast array of symbionts, and how it may regulate its metabolic quotient, for example through biochemical or 
epigenetic modification, will rapidly accelerate our ability to predict the fate of coral reefs.

\section{Availability and requirements}

ZoophyteBase was constructed using the Metagenome/ Genome Annotated Sequence Natural Language Search Engine (MEGGASENSE). This is a general system for the annotation of sequence collections and presentation of the results in a database that can be searched using biologically intuitive search terms. In this implementation, the predicted proteome of $A$. digitifera (genome assembly v1.0 [48]) was used as the source of protein sequences. The annotation was carried out using the KEGG database (release v58 [51]) to relate $A$. digitifera protein sequences to KEGG orthologues. The homologous protein sequences were used to construct hidden Markov model (HMM) profiles using the HMMER3 package [49]. The predicted proteome sequences of $A$. digitifera were searched with HMM profiles to link proteins to appropriate KEGG orthologues [50,512]. A web interface was developed with various tools. The search platform Lucene/Solr [52] was used to implement natural language searches. Protein sequences provided by the user can be used for BLAST [50] searches against the coral proteome. Selected sequences of the coral proteome can be analysed with third party software (e.g. [53]) to interrogate conserved domains. ZoophyteBase is deployed using Apache-Tomcat (version 7.0.28 for Linux $\times 64$ [513]) on the Ubuntu Linux server of the Section of Bioinformatics at the Faculty of Food Technology and Biotechnology, University of Zagreb, Croatia and is accessible at our published web address [47].

\section{Additional file}

Additional file 1: Table S16b. Predicted (UniProt) homologues of animal toxins encoded in the genome of A. digitifera.

\section{Competing interests}

The authors declare no competing interests exist.

\section{Authors' contributions}

WCD and PFL conceived the study, and participated in its design, coordination and drafted the manuscript. AS carried out the annotation. DB, JD, JZ and RG participated in the database design and testing. WCD, MJHVO, $\mathrm{AS}$ and PFL performed data analysis. $\mathrm{DH}$ and JC participated in and coordinated the annotation, database design and testing. All authors have read and approved the final manuscript.

\section{Acknowledgments}

The authors are indebted to Professor Noriyuki Satoh and his research team at the Marine Genomics Unit, Okinawa Institute of Science and Technology, Japan for making the protein sequence of A. digitifera publically available without restriction of use. We thank the following scientists from the Australian Institute of Marine Science for helpful discussions, Dr David Bourne, Dr Andrew Negri, Dr Elisha Wood-Charlson and Dr Karen Weynberg. We are also grateful to Dr Bill Leggat of the ARC Centre of Excellence for Coral Reef Studies and School of Pharmacy and Molecular Sciences, James Cook University, Townsville, Australia for critically reviewing this manuscript. Financial support for this work has come from the Biotechnology and
Biological Sciences Research Council of the United Kingdom (BBSRC grant BB/H010009/2 to WCD and PFL); from the Australian Institute of Marine Science (WCD and MvO); from a cooperation grant of the German Academic Exchange Service (DAAD) and Ministry of Science, Education and Sports, Republic of Croatia (to JC and DH) and from the Croatian Science Foundation (grant 09/5 to DH). The funders had no role in study design, data collection and analysis, decision to publish, or preparation of the manuscript. Initial work (in progress) was presented at the $12^{\text {th }}$ International Coral Reef Symposium, Cairns, Queensland, Australia, 9-13 July 2012.

\section{Author details}

${ }^{1}$ Centre for Marine Microbiology and Genetics, Australian Institute of Marine Science, PMB No. 3 Townsville MC, Townsville 4810, Queensland, Australia. ${ }^{2}$ Institute of Pharmaceutical Science, King's College London, Franklin-Wilkins Building, 150 Stamford Street, London SE1 9NH, United Kingdom.

${ }^{3}$ Department of Chemistry King's College London, Franklin-Wilkins Building, 150 Stamford Street, London SE1 9NH, United Kingdom. ${ }^{4}$ Section for Bioinformatics, Department of Biochemical Engineering, Faculty of Food Technology and Biotechnology, University of Zagreb, Pierottijeva 6, 10000 Zagreb, Croatia. ${ }^{5}$ Department of Genetics, University of Kaiserslautern, Postfach 3049, 67653 Kaiserslautern, Germany.

Received: 6 March 2013 Accepted: 15 July 2013

Published: 26 July 2013

\section{References}

1. Freudenthal HD: Symbiodinium gen. nov. and Symbiodinium microadriaticum sp. nov., a zooxanthella: taxonomy, life cycle, and morphology. J Eukaryot Microbiol 1962, 9:45-52.

2. Muscatine $L$ : The role of symbiotic algae in carbon and energy flux in reef corals. In Coral Reefs: Ecosystems of the World. Edited by Dubinsky Z. Amsterdam: Elsevier; 1990:75-84.

3. Yellowlees D, Rees TAV, Leggat W: Metabolic interactions between algal symbionts and invertebrate hosts. Plant Cell Environ 2008, 31:679-694.

4. Johannes RE, Wiebe WJ, Crossland CJ, Rimmer DW, Smith SV: Latitudinal limits of coral reef growth. Mar Ecol Prog Ser 1983, 11:105-111.

5. Spalding MD, Grenfell AM: New estimates of global and regional coral reef areas. Coral Reefs 1997, 16:225-230.

6. Hatcher BG: Coral reef primary productivity. A hierarchy of pattern and process. Trends Ecol Evol 1990, 5:149-155.

7. Reaka-Kudla ML: The global biodiversity of coral reefs: A comparison with rainforests. In Biodiversity II. Understanding and Protecting Our Natural Resources. Edited by Reaka-Kudla ML, Wilson DE, Wilson EO. Washington DC: Joseph Henry/National Academy Press; 1997:83-108.

8. Davy SK, Allemand D, Weis VM: Cell biology of cnidarians-dinoflagellate symbiosis. Microbiol Mol Biol Rev 2012, 76:229-261.

9. Bellwood DR, Hughes TP, Folke C, Nyström M: Confronting the coral reef crisis. Nature 2004, 429:827-833.

10. Hoegh-Guldberg O: Climate change, coral bleaching and the future of the world's coral reefs. Mar Freshwat Res 1999, 50:839-866.

11. Hoegh-Guldberg O, Mumby PJ, Hooten AJ, Steneck RS, Greenfield P, Gomez E, Harvell CD, Sale PF, Edwards AJ, Caldeira K, Knowlton N, Eakin CM, Iglesias-Prieto R, Muthiga N, Bradbury RH, Dubi A, Hatziolos ME: Coral reefs under rapid climate change and ocean acidification. Science 2007, 318:1737-1742.

12. Porter JW, Dustan $P$, Jaap WC, Patterson $K L$, Kosmynin V, Meier OW, Patterson ME, Parsons M: Patterns of spread of coral disease in the Florida Keys. Hydrobiologia 2001, 460:1-24.

13. Bruno JF, Selig ER, Casey KS, Page CA, Willis BL, Harvell CD, Sweatman H, Melendy AM: Thermal stress and coral cover as drivers of coral disease outbreaks. PLOS Biol 2007, 5:e124

14. Brandt ME, McManus JW: Disease incidence is related to bleaching extent in reef-building corals. Ecology 2009, 90:2859-2867.

15. Devlin MJ, Brodie J: Terrestrial discharge into the Great Barrier Reef Lagoon: nutrient behaviour in coastal waters. Mar Pollut Bull 2005, 51:9-22.

16. Fabricius KE: Effects of terrestrial runoff on the ecology of corals and coral reefs: review and synthesis. Mar Pollut Bull 2005, 50:125-146.

17. Orr JC, Fabry VJ, Aumont O, Bopp L, Doney SC, Feely RA, Gnanadesikan A, Gruber N, Ishida A, Joos F, Key RM, Lindsay K, Maier-Reimer E, Matear R, 
Monfray P, Mouchet A, Najjar RG, Plattner GK, Rodgers KB, Sabine CL, Sarmiento JL, Schlitzer R, Slater RD, Totterdell IJ, Weirig MF, Yamanaka Y, Yool A: Anthropogenic ocean acidification over the twenty-first century and its impact on calcifying organisms. Nature 2005, 437:681-686.

18. Anthony KRN, Kline DI, Diaz-Pulido G, Dove S, Hoegh-Guldberg O: Ocean acidification causes bleaching and productivity loss in coral reef builders. Proc Natl Acad Sci U S A 2008, 105:17442-17446.

19. De'ath $G$, Fabricius K: Water quality as a regional driver of coral biodiversity and macroalgae on the Great Barrier Reef. Ecol App/ 2010, 20:840-850

20. Carpenter KE, Abrar M, Aeby G, Aronson RB, Banks S, Bruckner A, Chiriboga A, Cortés J, Delbeek JC, Devantier L, Edgar GJ, Edwards AJ, Fenner D, Guzmán HM, Hoeksema BW, Hodgson G, Johan O, Licuanan WY, Livingstone SR, Lovell ER, Moore JA, Obura DO, Ochavillo D, Polidoro BA, Precht WF, Quibilan MC, Reboton C, Richards ZT, Rogers AD, Sanciangco J, et al: One third of reef-building corals face elevated extinction risk from climate change and local impacts. Science 2008, 321:560-563.

21. Pendleton LH: Valuing coral reef protection. Ocean Coast Manage 1995 26:119-131

22. Halpern BS, Walbridge S, Selkoe KA, Kappel CV, Micheli F, D'Agrosa C, Bruno JF, Casey KS, Ebert C, Fox HE, Fujita R, Heinemann D, Lenihan HS, Madin EM, Perry MT, Selig ER, Spalding M, Steneck R, Watson R: A global map of human impact on marine ecosystems. Science 2008, 319:948-952.

23. Hughes TP, Baird AH, Bellwood DR, Card M, Connolly SR, Folke C, Grosberg R, Hoegh-Guldberg O, Jackson JB, Kleypas J, Lough JM, Marshall P, Nyström M, Palumbi SR, Pandolfi JM, Rosen B, Roughgarden J: Climate change, human impacts, and the resilience of coral reefs. Science 2003, 301:929-933.

24. Evans TG, Hofmann GE: Defining the limits of physiological plasticity: how gene expression can assess and predict the consequences of ocean change. Philos Trans R. Soc B-Biol Sci 2012, 367:1733-1745.

25. Weis VM: The susceptibility and resilience of corals to thermal stress: adaptation, acclimatization or both? Mol Ecol 2010, 19:1515-1517.

26. Edge SE, Morgan MB, Gleason DF, Snell TW: Development of a coral cDNA array to examine gene expression profiles in Montastraea faveolata exposed to environmental stress. Mar Pollut Bull 2005, 51:507-523.

27. Grasso LC, Maindonald J, Rudd S, Hayward DC, Saint R, Miller DJ, Ball EE: Microarray analysis identifies candidate genes for key roles in coral development. BMC Genomics 2008, 9:540.

28. Bay LK, Ulstrup KE, Nielsen HB, Jarmer H, Goffard N, Willis BL, Miller DJ van Oppen MJ: Microarray analysis reveals transcriptional plasticity in the reef building coral Acropora millepora. Mol Ecol 2009, 18:3062-3075.

29. Meyer E, Aglyamova GV, Wang S, Buchanan-Carter J, Abrego D, Colbourne $\mathrm{JK}$, Willis BL, Matz MV: Sequencing and de novo analysis of a coral larval transcriptome using 454 GSFlx. BMC Genomics 2009, 10:219.

30. DeSalvo MK, Sunagawa S, Voolstra CR, Medina M: Transcriptomic responses to heat stress and bleaching in the elkhorn coral Acropora palmata. Mar Ecol Prog Ser 2010, 402:97-113.

31. Portune KJ, Voolstra CR, Medina M, Szmant AM: Development and heat stress induced transcriptomic changes during embryogenesis of the scleractinian coral Acropora palmata. Mar Genom 2010, 3:51-62.

32. Souter P, Bay LK, Andreakis N, Császár N, Seneca FO, van Oppen MJ: A multilocus, temperature stress-related gene expression profile in Acropora millepora, a dominant reef-building coral. Mol Ecol Resour 2011, 11:328-334.

33. Ladner JT, Barshis DJ, Palumbi SR: Protein evolution in two co-occurring types of Symbiodinium: an exploration into the genetic basis of thermal tolerance in Symbiodinium clade D. BMC Evol Biol 2012, 12:217.

34. Barshis DJ, Ladner JT, Oliver TA, Seneca FO, Traylor-Knowles N, Palumbi SR: Genomic basis for coral resilience to climate change. Proc Natl Acad Sci USA 2013, 110:1387-1392.

35. Granados-Cifuentes C, Bellantuono AJ, Ridgway T, Hoegh-Guldberg O, Rodriguez-Lanetty M: High natural gene expression variation in the reef-building coral Acropora millepora: potential for acclimative and adaptive plasticity. BMC Genomics 2013, 14:228.

36. Traylor-Knowles N, Granger BR, Lubinski TJ, Parikh JR, Garamszegi S, Xia Y, Marto JA, Kaufman L, Finnerty JR: Production of a reference transcriptome and transcriptomic database (PocilloporaBase) for the cauliflower coral, Pocillopora damicornis. BMC Genomics 2011, 12:585.
37. Sun J, Chen Q, Lun JC, Xu J, Qiu JW: PcarnBase: Development of a transcriptomic database for the brain coral Platygyra carnosus. Mar Biotechnol 2013, 15:244-451.

38. Cossins A, Fraser J, Hughes M, Gracey A: Post-genomic approaches to understanding the mechanisms of environmentally induced phenotypic plasticity. J Exp Biol 2006, 209:2328-2336.

39. Weston AJ, Dunlap WC, Shick JM, Klueter A, Iglic K, Vukelic A, Starcevic A, Ward M, Wells ML, Trick CG, Long PF: A profile of an endosymbiontenriched fraction of the coral Stylophora pistillata reveals proteins relevant to microbial-host interactions. Mol Cell Proteomics 2012, 11: M111.015487

40. Starcevic A, Dunlap WC, Cullum J, Shick JM, Hranueli D, Long PF: Gene expression in the scleractinian Acropora microphthalma exposed to high solar irradiance reveals elements of photoprotection and coral bleaching. PLoS One 2010, 5:e13975.

41. Miller DJ, Ball EE, Technau U: Cnidarians and ancestral genetic complexity in the animal kingdom. Trends Genet 2005, 21:536-539.

42. Putnam NH, Srivastava M, Hellsten U, Dirks B, Chapman J, Salamov A Terry A, Shapiro H, Lindquist E, Kapitonov W, Jurka J, Genikhovich G, Grigoriev IV, Lucas SM, Steele RE, Finnerty JR, Technau U, Martindale MQ, Rokhsar DS: Sea anemone genome reveals ancestral eumetazoan gene repertoire and genomic organization. Science 2007, 317:86-94.

43. Kortschak RD, Samuel G, Saint R, Miller DJ: EST analysis of the cnidarians Acropora millepora reveals extensive gene loss and rapid sequence divergence in the model invertebrates. Curr Biol 2003, 13:2190-2195.

44. Chapman JA, Kirkness EF, Simakov O, Hampson SE, Mitros T, Weinmaier T, Rattei T, Balasubramanian PG, Borman J, Busam D, Disbennett K, Pfannkoch C, Sumin N, Sutton GG, Viswanathan LD, Walenz B, Goodstein DM, Hellsten U, Kawashima T, Prochnik SE, Putnam NH, Shu S, Blumberg B, Dana CE, Gee L, Kibler DF, Law L, Lindgens D, Martinez DE, Peng J, Wigge PA, et al: The dynamic genome of the Hydra. Nature 2010, 464:591-596.

45. Shinzato C, Shoguchi E, Kawashima T, Hamada M, Hisata K, Tanaka M, Fujie M, Fujiwara M, Koyanagi R, Ikuta T, Fujiyama A, Miller DJ, Satoh N: Using the Acropora digitifera genome to understand coral responses to environmental change. Nature 2011, 476:320-323.

46. Coral genome sequence data usage policy. [http://coralbase.org/accounts/ register/]

47. ZoophyteBase coral proteome database. [http://bioserv7.bioinfo.pbf.hr/ Zoophyte/index.jsp]

48. Genome Sequencing/Annotation Projects. [http://marinegenomics.oist.jp/ genomes/download?\%20project_id=3]

49. Finn RD, Clements J, Eddy SR: HMMER web server: interactive sequence similarity searching. Nucleic Acids Res 2011, 39(Web Server Issue):W29-W37.

50. Camacho C, Coulouris G, Avagyan V, Ma N, Papadopoulos J, Bealer K, Madden TL: BLAST+: architecture and applications. BMC Bioinforma 2009, $10: 421$.

51. KEGG: Kyoto Encyclopedia of Genes and Genomes. [http://www.genome.jp/ $\mathrm{kegg} / \mathrm{J}$

52. Apache Solr ${ }^{\mathrm{Tm}}$. [http://lucene.apache.org/solr/

53. Conserved Domain Database(CDD). [http://www.ncbi.nlm.nih.gov/Structure/ $\mathrm{cdd} / \mathrm{cdd}$.shtml]

54. Stanley GD Jr: Photosymbiosis and the evolution of modern coral reefs. Science 2006, 312:857-858.

55. Dubinski Z, Falkowski P: Light as a source of information and energy in zooxanthellate corals. In Coral Reefs: And Ecosystem in Transition. Edited by Dubinski Z, Stambler N. Berlin: Springer-Verlag; 2011:107-118.

56. Dunn SR, Schnitzler CE, Wies VM: Apoptosis and autophagy as mechanisms of dinoflagellate symbiont release during cnidarian bleaching: every which way you lose. Proc Biol Soc 2007, 274:3079-3085

57. Downs CA, Kramarsky-Winter E, Martinez J, Kushmaro A, Woodley CM, Loya Y, Ostrander GK: Symbiophagy as a cellular mechanism for coral bleaching. Autophagy 2009, 5:211-216.

58. Muscatine $L$, Pool RR: Regulation of numbers of intracellular alage. Proc $R$ Soc B 1979, 204:131-139.

59. Hutagalung $A H$, Novick PJ: Role of Rab GTPases in membrane traffic and cell physiology. Physiol Rev 2011, 91:119-149.

60. Hong M-C, Huang Y-S, Lin W-W, Fang L-S, Chen M-C: ApRab3, a biosynthetic Rab protein, accumulates on the maturing phagosomes and symbiosomes in the tropical sea anemone, Aiptasia pulchella. Comp Biochem Physiol B Biochem Mol Biol 2009, 152:249-259. 
61. Hong M-C, Huang J-S, Song P-C, Lin W-W, Fang L-S, Chen M-C: Cloning and characterization of ApRab4, a recycling Rab protein of Aiptasia pulchella, and its implication in the symbiosome biogenesis. Mar Biotechnol 2009, 11:771-785.

62. Chen MC, Cheng YM, Hong MC, Fang LS: Molecular cloning of Rab5 (ApRab5) in Aiptasia pulchella and its retention in phagosomes harbouring live zooxanthellae. Biochem Biophys Res Commun 2004, 324:1024-1033.

63. Chen MC, Cheng YM, Sung PJ, Kuo CE, Fang LS: Molecular identification of Rab7 (ApRab7) in Aiptasia pulchella and its exclusion from phagosomes harboring zooxanthellae. Biochem Biophys Res Commun 2003, 308:586-595.

64. Chen MC, Hong MC, Huang YS, Liu MC, Cheng YM, Fang LS: ApRab11, a cnidarian homologue of the recycling regulatory protein Rab11, is involved in the establishment and maintenance of the Aiptasia-Symbiodinium endosymbiosis. Biochem Biophys Res Commun 2005, 338:1607-1616

65. Collins RN: Rab and ARF GTPase regulation of exocytosis. Mol Membr Biol 2003, 20:105-115.

66. Deneka M, Neeft M, van der Sluijs P: Regulation of membrane transport by Rab GTPases. Crit Rev Biochem Mol Biol 2003, 38:121-142.

67. Vassilieva EV, Nusrat A: Vesicular trafficking: molecular tools and targets. Methods Mol Biol 2008, 440:3-14

68. Ohya T, Miaczynska M, Coskun Ü, Lommer B, Runge A, Drechsel D, Kalaidzidis Y, Zerial M: Reconstruction of Rab- and SNARE-dependent membrane fusion by synthetic endosomes. Nature 2009, 459:1091-1097.

69. SNARE Database. [http://bioinformatics.mpibpc.mpg.de/snare/ snareQueryPage.jsp]

70. Kloepper TH, Kienle CN, Fasshauer D: An elaborate classification of SNARE proteins sheds light on the conservation of the eukaryotic endomembrane system. Mol Biol Cell 2007, 18:3463-3471.

71. Hay JC, Chao DS, Kuo CS, Scheller RH: Protein interactions regulating vesicle transport between the endoplasmic reticulum and Golgi apparatus in mammalian cells. Cell 1997, 89:149-158.

72. Masuda ES, Huang BC, Fisher JM, Luo Y, Scheller RH: Tomosyn binds tSNARE proteins via a VAMP-like coiled coli. Neuron 1998, 21:479-480.

73. Scales SJ, Hesser BA, Masuda ES, Scheller RH: Amisyn, a novel syntaxin-binding protein that may regulate SNARE complex assembly. $J$ Biol Chem 2002, 227:28271-28279.

74. Jahn R, Scheller RH: SNAREs - Engines for membrane fusion. Nat Rev Mol Cell Biol 2006, 7:631-643.

75. Muscatine L, Pool RR, Cernichiari E: Some factors influencing selective release of soluble organic material by zooxanthellae from reef corals. Mar Biol 1972, 13:298-308.

76. Sutton DC, Hoegh-Guldberg O: Host-zooxanthellae interactions in four temperate marine invertebrate symbioses: assessment of the effect of host extracts on symbionts. Biol Bull 1990, 178:175-186.

77. Masuda K, Miyachi S, Maruyama T: Sensitivity of zooxanthellae and nonsymbiotic microalgae to stimulation of photosynthate excretion by giant clam tissue homogenate. Mar Biol 1994, 118:687-693.

78. Narayanan A, Nogueira ML, Ruyechan WT, Kristie TM: Combinatorial transcription of Herpes simplex virus and Varicella zoster virus intermediate early genes is strictly determined by cellular coactivator HCF-1. J Biol Chem 2005, 280:1369-1375.

79. Lee S, Horn V, Julien E, Liu Y, Wysocka J, Bowerman B, Hengartner MO, Herr W: Epigenetic regulation of histone $\mathrm{H} 3$ serine 10 phosphorylation status by HCF-1 proteins in C. elegans and mammalian cells. PLoS One 2007, 2:e1213.

80. Kristie TM, Liang Y, Vogel JL: Control of a-herpesvirus IE gene expression by HCF-1 coupled chromatin modification activities. Biochem Biophys Acta 2010, 1799:257-265.

81. Christensen RG, Enuameh MS, Noyes MB, Brodsky MH, Wolfe SA, Stormo GD: Recognition models to predict DNA-binding specificities of homeodomain proteins. Bioinformatics 2012, 28:i84-i89.

82. Mann RS, Lelli KM, Joshi R: Hox specificity: unique roles for cofactors and collaborators. Curr Top Dev Biol 2009, 88:66-101.

83. Finnerty JR, Martindale MQ: Ancient origins of axial patterning genes: Hox genes and ParaHox genes in the Cnidaria. Evol Dev 1999, 1:16-23.

84. Hislop NR, de Jong D, Hayward DC, Ball EE, Miller DJ: Tandem organisation of independently duplicated homeobox genes in the basal cnidarian Acropora millepora. Dev Genes Evol 2005, 215:268-273.

85. Ryan JF, Mazza ME, Pang K, Matus DQ, Baxevanis AD, Martindale MQ, Finnerty JR: Pre-bilaterian origins of the Hox cluster and the Hox code: evidence from the sea anemone, Nematostella vectensis. PLoS One 2007, 2:e153.
86. Larroux C, Fahey B, Degnan SM, Adamski M, Rokhsar DS, Degnan BM: The NK homeobox gene cluster predates the origin of Hox genes. Curr Biol 2007, 17:706-710.

87. Dowid IB, Chitnis AB: LIM homeobox genes and the CNS: a close relationship. Neuron 2001, 30:301-303.

88. Srivastava M, Larroux C, Lu CD, Mohanty K, Chapman J, Degnan BM, Rokhsa DS: Early evolution of the LIM homeobox gene family. BMC Biol 2010, 8:4.

89. Ryan JF, Burton PM, Mazza ME, Kwong GK, Mullikin JC, Finnerty JR: The cnidarian-bilaterian ancestor possessed at least 56 homeoboxes: evidence from the starlet sea anemone, Nematostella vectensis. Genome Biol 2006, 7:R64.

90. Saito T, Sawamoto K, Okano H, Anderson DJ, Mikoshiba K: Mammalian BarH homologue is a potential regulator of neural bHLH genes. Dev Biol 1998, 199:216-225.

91. Habas $S$, Kato $Y$, He X: Wnt/Frazzled activation of Rho regulates vertebrate gastrulation and requires a novel Formin homology protein Daam1. Cell 2001, 107:843-854.

92. Matusek T, Djiane A, Jankovics F, Brunner D, Mlodzik M, Mihály J: The Drosophila formin DAAM regulates the tracheal cuticle pattern through organizing the actin cytoskeleton. Development 2006, 133:957-966.

93. Li D, Hallett MA, Zhu W, Rubart M, Liu Y, Yang Z, Chen H, Haneline LS, Chan RJ, Schwartz RJ, Field LJ, Atkinson SJ, Shou W: Dishevelled-associated activator of morphogenesis 1 (Daam1) is required for heart morphogenesis. Development 2011, 138:303-315.

94. Wada Y, Kitamoto K, Kanbe T, Tanaka K, Anraku Y: The SLP1 gene of Saccharomyces cerevisiae is essential for vacuolar morphogenesis and function. Mol Cell Biol 1990, 10:2214-2223.

95. Bragdon B, Moseychuk O, Saldanha S, King D, Julian J, Nohe A: Bone morphogenetic proteins: a critical review. Cell Signal 2011, 23:609-620.

96. Martin VJ: Photoreceptors of cnidarians. Can J Zool 2002, 80:1703-1722.

97. Mason BM, Cohen $\mathrm{JH}$ : Long-wavelength photosensitivity in coral planula larvae. Biol Bull 2012, 222:88-92.

98. Gorbunov MY, Falkowski PG: Photoreceptors in the cnidarian host allow symbiotic corals to sense blue moonlight. Limnol Oceanogr 2002, 47:309-315.

99. Levy O, Appelbaum L, Leggat W, Gothlif Y, Hayward DC, Miller DJ, Hoegh-Guldberg O: Light-responsive cryptochromes from a simple multicellular animal, the coral Acropora millepora. Science 2007, 318:467-470.

100. Vize PD: Transcriptome analysis of the circadian regulatory network in the coral Acropora millepora. Biol Bull 2009, 216:131-137.

101. Brady AK, Snyder KA, Vize PD: Circadian cycles of gene expression in the coral, Acropora millepora. PLoS One 2011, 6:e25072.

102. Ishiura M, Kutsuna S, Aoki S, Iwasaki H, Andersson CR, Tanabe A, Golden SS, Johnson $\mathrm{CH}$, Kondo T: Expression of a gene cluster kaiABC as a circadian feedback process in cyanobacteria. Science 1998, 281:1519-1523.

103. Nishiwaki T, Satomi Y, Nakajima M, Lee C, Kiyohara R, Kageyama H, Kitayama Y, Temamoto M, Yamaguchi A, Hijikata A, Go M, Iwasaki H, Takao T, Kondo $\mathrm{T}$ : Role of KaiC phosphorylation in the circadian clock system of Synechococcus elongatus PCC 7942. Proc Natl Acad Sci USA 2004, 101:13927-13932.

104. Xu Y, Mori T, Qin X, Yan H, Egli M, Johnson CH: Intramolecular regulation of phosphorylation status of the circadian clock protein KaiC. PLOS One 2009, 4:e7509.

105. Nakahira Y, Katayama M, Miyashita H, Katsuna S, Iwasaki H, Oyama T, Kondo T: Global gene repression by KaiC as a master process of prokaryotic circadian system. Proc Natl Acad Sci USA 2004, 101:881-885.

106. Kondo T, Ishiura M: The circadian clock of cyanobacteria. Bioessays 2000, 22:10-15.

107. Hardin PE, Hall JC, Rosbash M: Feedback of the Drosophila period gene product on circadian cycling of its messenger RNA levels. Nature 1990 343:536-540.

108. Dunlap JC: Molecular bases for circadian clocks. Cell 1999, 96:271-290.

109. Reitzel AM, Behrendt L, Tarrant AM: Light entrained rhythmic gene expression in the sea anemone Nematostella vectensis: the evolution of the animal circadian clock. PLoS One 2010, 5:e12805.

110. Gibbons IR: Cilia and flagella of eukaryotes. J Cell Biol 1981, 91:107s-124s.

111. Linck RW: Tektins and microtubules. Adv Mol Cell Biol 1990, 3:35-63.

112. Fox LA, Sawin KE, Sale WS: Kinesin-related proteins in eukaryotic flagella. J Cell Sci 1994, 107:1545-1550.

113. Amos $L A$ : The tektin family of microtubule-stabilizing proteins. Genome Biol 2008, 9:229. 
114. Kozminski KG, Beech PL, Rosenbaum JL: The Chlamydomonas kinesin-like protein FLA10 is involved in motility associated with the flagellar membrane. J Cell Biol 1995, 131:1517-1527.

115. Bartlett DH, Frantz BB, Matsumura P: Flagellar activators FlbB and Flal: gene sequences and $5^{\prime}$ consensus sequences of operons under $\mathrm{FlbB}$ and Flal control. J Bacteriol 1988, 170:1575-1581.

116. Aldridge P, Karlinsey J, Hughes KT: The type III secretion chaperone FlgN regulates flagellar assembly via a negative feedback loop containing its chaperone substrates FlgK and FlgL. Mol Microbiol 2003, 49:1333-1345.

117. Ferris HU, Furukawa Y, Minamino T, Kroetz MB, Kihara M, Namba K, Macnab RM: FlhB regulates ordered export of flagellar components via autocleavage mechanisms. J Biol Chem 2005, 280:41236-41242.

118. Mattick JS: Type IV pili and twitching motility. Annu Rev Microbiol 2002, 56:289-314.

119. Starcevic A, Akthar S, Dunlap WC, Shick JM, Hranueli D, Cullum J, Long PF: Enzymes of the shikimate acid pathway encoded in the genome of a basal metazoan, Nematostella vectensis, have microbial origins. Proc Natl Acad Sci U S A 2008, 105:2533-2537.

120. Rebbapragada A, Johnson MS, Harding GP, Zuccarelli AJ, Fletcher HM, Zhulin IB, Taylor BL: The Aer protein and the serine chemoreceptor Tsr independently sense intracellular energy levels and transduce oxygen, redox, and energy signals for Escherichia coli behavior. Proc Natl Acad Sci USA 1997, 94:10541-10546.

121. Troemei ER, Chou JH, Dwyer ND, Colbert HA, Bargmann Cl: Divergent seven transmembrane receptors are candidate chemosensory receptors in C. elegans. Cell 1995, 83:207-218.

122. Sen M, Shah A, Marsh L: Two types of alpha-factor receptor determinants for pheromone specificity in the mating-incompatible yeasts $S$. cerevisiae and S. kluyveri. Curr Genet 1997, 31:235-240.

123. Gestwicki JE, Lamanna AC, Harshey RM, McCarter LL, Kiessling LL, Adler J: Evolutionary conservation of methyl accepting chemotaxis protein location in Bacteria and Archaea. J Bacteriol 2000, 182:6499-6502.

124. Capra EJ, Laub MT: Evolution of two-component signal transduction systems. Annu Rev Microbiol 2012, 66:325-347.

125. Koretke KK, Lupas AN, Warren PV, Rosenberg M, Brown JR: Evolution of two-component signal transduction. Mol Biol Evol 2000, 17:1956-1970.

126. DeVries ME, Kelvin AA, Xu L, Ran L, Robertson J, Kelvin DJ: Defining the origins and evolution of the chemokine/chemokine receptor system. $\mathrm{J}$ Immunol 2006, 176:401-415.

127. Holland ND: Early central nervous system evolution: an era of skin brains? Nat Rev Neurosci 2003, 4:617-627.

128. Galliot B, Quiquand M, Ghila L, de Rosa R, Miljkovic-Licina M, Chera S: Origins of neurogenesis, a cnidarian view. Dev Biol 2009, 332:2-24.

129. Nakanishi N, Renfer E, Technau U, Rentzsch F: Nervous systems of the sea anemone Nematostella vectensis are generated by ectoderm and endoderm and shaped by distinct mechanisms. Development 2012, 139:347-357.

130. Sakarya O, Armstrong KA, Adamska M, Adamski M, Wang IF, Tidor B, Degnan BM, Oakley TH, Kosik KS: A post-synaptic scaffold at the origin of the animal kingdom. PLoS One 2007, 2:e506.

131. Miljkovik-Licina M, Gauchat D, Galliot B: Neuronal evolution: analysis of regulatory genes in a first-evolved nervous system, the hydra nervous system. Biosystems 2004, 76:75-87.

132. Marlow HQ, Srivastava M, Matus DQ, Rokhsar D, Martindale MQ: Anatomy and development of the nervous system of Nematostella vectensis, an anthozoan cnidarian. Dev Neurobiol 2009, 69:235-254.

133. Kass-Simon G, Pierobon P: Cnidarian chemical neurotransmission, an updated overview. Comp Biochem Physiol A Mol Intergr Physiol 2007 146:9-25.

134. Grimmelikhuijzen CJP, Westfall JA: The nervous systems of cnidarians. In The Nervous Systems of Invertebrates. An Evolutionary and Comparative Approach. Edited by Breidbach O, Kutch W. Basel: Brikhäuser Verlag; 1995:7-24

135. Sleeman MW, Anderson KD, Lambert PD, Yancopoulos GD, Wiegand SJ: The ciliary neurotrophic factor and its receptor, CNFRTA alpha. Pharm Acto Helv 2000, 74:265-272.

136. Kass-Simon G, Scappaticci AA Jr: The behavioural and developmental physiology of nematocysts. Can J Zool 2002, 80:1772-1794.

137. Plachetzki DC, Fong CR, Oakley TH: Cnidocyte discharge is regulated by light and opsin-mediated phototransduction. BMC Biol 2012, 10:17.

138. Coates MM: Visual ecology and functional morphology of Cubozoa (Cnidaria). Integr Comp Biol 2003, 43:542-548.
139. Koyanagi M, Takano K, Tsukamoto H, Ohtsu K, Tokunaga F, Terakita A: Jellyfish vision starts with CAMP signalling mediated by opsin- $\mathrm{G}_{\mathrm{S}}$ cascade. Proc Natl Acad Sci U S A 2008, 105:15576-15580.

140. Shichida $Y$, Matsuyama T: Evolution of opsins and phototransduction. Philos Trans R Soc Lond B Biol Sci 2009, 364:2881-2895.

141. Beliaev A, Learmonth DA, Soares-da-Silva P: Synthesis and biological evaluation of novel, peripherally selective chromanyl imidazolethione-based inhibitors of dopamine beta-hydroxylase. J Med Chem 2006, 49:1191-1197.

142. Grimmelikhuijzen CJP, Williamson M, Hansen GN: Neuropeptides in cnidarians. Can J Zool 2002, 80:1690-1702.

143. Attenborough RM, Hayward DC, Kitahara MV, Miller DJ, Ball EE: A "neural" enzyme in nonbilaterian animals and algae: preneural origins for peptidylglycine a-amindating monooxygenase. Mol Biol Evol 2012, 29:3095-3109.

144. Allemand D, Tambutté E, Zoccola D, Tambutté S: Coral calcification, cells to reefs. In Coral Reefs: An Ecosystem in Transition. Edited by Dubinsky Z, Stambler N. Dordrecht: Springer; 2011:119-150.

145. Bénazet-Tambutté S, Allemand D, Joubert J: Permeability of the oral epithelial layers in cnidarians. Mar Biol 1996, 126:43-53.

146. Al-Horani FA, Al-Moghrabi SM, de Beer D: The mechanism of calcification and its relation to photosynthesis and respiration in the scleractinian coral Galaxea fascicularis. Mar Biol 2003, 142:419-429.

147. Zoccola D, Tambutté E, Sénégas-Balas F, Michiels JF, Failla JP, Jaubert J, Allemand D: Cloning of a calcium channel a1 subunit from the reef-building coral, Stylophora pistillata. Gene 1999, 227:157-167.

148. Zoccola D, Tambutté E, Kulhanek E, Puverel S, Scimeca JC, Allemand D, Tambutté S: Molecular cloning and localisation of a PMCA P-type calcium ATPase from the coral Stylophora pistillata. Biochim Biophys Acta 2004, 1663:117-126.

149. Moya A, Tambutté S, Bertucci A, Tambutté E, Lotto S, Vullo D, Supuran CT, Allemand D, Zoccola D: Carbonic anydrase in the scleractinian coral Stylophora pistillata: characterisation, localisation, and role in biomineralisation. J Biol Chem 2008, 283:25475-25484.

150. Gattuso J-P, Allemand D, Frankignoulle M: Photosynthesis and calcification at cellular, organismal and community levels in coral reefs: A review on interactions and control by carbonate chemistry. Amer Zool 1999, 39:160-183.

151. Allemand D, Ferrier-Pagè $C$, Furla $P$, Houlbrèque F, Puverel $S$, Reynaud $S$, Tambutté É, Tambutté S, Zoccola D: Biomineralisation in reef-building corals: from molecular mechanisms to environmental control. C R Palevol 2004, 3:453-467.

152. Venn A, Tambutté E, Holcomb M, Allemand D, Tambutté S: Live tissue imaging shows corals elevate $\mathrm{pH}$ under their calcifying tissues relative to seawater. PLoS One 2011, 6:e20013.

153. Kaniewska P, Campbell PR, Kline DI, Rodriguez-Lanetty M, Miller DJ, Dove S, Hoegh-Guldberg O: Major cellular and physiological impacts of ocean acidification on a reef building coral. PLoS One 2012, 7:e34659.

154. Catterall WA: Voltage-gated calcium channels. Cold Spring Harb Perspect Biol 2011, 3:003947.

155. Bertucci A, Tembutté S, Supuran CT, Allemand D, Zoccola D: A new coral carbonic anhydrase in Stylophora pistillata. Mar Biotechnol 2011, 13:992-1002.

156. Morita M, Iguchi A, Takamura A: Roles of calmodulin and calcium/ calmodulin-dependent protein in flagellate motility regulation in the coral Acropora digitifera. Mar Biotechnol 2009, 11:118-123.

157. Lane CE, Archibald JM: The eukaryotic tree of life: endosymbionts takes its TOL. Trends Ecol Evol 2008, 32:268-275

158. Keeling PJ: Functional and ecological impacts of horizontal gene transfer in eukaryotes. Curr Opin Genet Dev 2009, 19:613-619.

159. Bock R: The give-and-take of DNA: horizontal gene transfer in plants. Trends Plant Sci 2010, 15:11-22

160. Balskus EP, Walsh CT: The genetic and molecular basis for sunscreen biosynthesis in cyanobacteria. Science 2010, 329:1653-1656.

161. Waller RF, Stamovits CH, Keeling PJ: Lateral gene transfer of a multigene region from cyanobacteria to dinoflagellates resulting in a novel plastid-targeted fusion protein. Mol Biol Evol 2006, 23:1437-1443.

162. Richards TA, Dacks JB, Campbell SA, Blanchard JL, Foster PG, McLeod R, Roberts CW: Evolutionary origins of the eukaryotic shikimate pathway: gene fusions, horizontal transfer, and endosymbiotic replacement. Eukaryot Cell 2006, 5:1517-1531

163. Habetha M, Bosch TC: Symbiotic Hydra express a plant-like peroxidase gene during oogenesis. J Exp Biol 2005, 208:2157-2165. 
164. Technau U, Miller MA, Bridge D, Steele RE: Arrested apoptosis of nurse cells during Hydra oogenesis and embryogenesis. Dev Biol 2003, 260:191-206.

165. Rumpho ME, Worful JM, Lee J, Kannan K, Tyler MS, Bhattacharya D, Moustafa A, Manhart JR: Horizontal gene transfer of the algal nuclear gene $p s b O$ to the photosynthetic sea slug Elysia chlorotica. Proc Natl Acad Sci U S A 2008, 105:17867-17871.

166. Rumpho ME, Pelletreau KN, Moustafa A, Bhattacharya D: The making of a photosynthetic animal. J Exp Biol 2011, 214:303-311.

167. Pierce SK, Curtis NE: Cell biology of the chloroplast symbiosis in sacaglossan sea slugs. Int Rev Cell Mol Biol 2012, 293:123-148.

168. Wägele H, Deusch O, Händeler K, Martin R, Schmitt V, Christa G, Pinzger B, Gould SB, Dagan T, Klussmann-Kolb A, Martin W: Transcriptomic evidence that longevity of acquired plastids in the photosynthetic slugs Elysia timida and Plakobranchus ocellatus does not entail lateral transfer of algal nuclear genes. Mol Biol Evol 2011, 28:699-706.

169. Pierce SK, Fang X, Schwartz JA, Jiang X, Zhao W, Curtis NE, Kocot KM, Yang $B$, Wang J: Transcriptomic evidence for the expression of horizontally transferred algal nuclear genes in the photosynthetic sea slug, Elysia chlorotica. Mol Biol Evol 2012, 29:1545-1556

170. Lang BF, O'Kelly C, Nerad T, Gray MW, Burger G: The closest unicellular relatives of animals. Curr Biol 2002, 12:1773-1778.

171. Ruiz-Trillo I, Roger AJ, Burger G, Gray MW, Lang BF: A phylogenomic investigation into the origins of metazoa. Mol Biol Evol 2008, 25:664-672.

172. Sun G, Yang Z, Ishwar A, Huang J: Algal genes in the closest relatives of animals. Mol Biol Evol 2010, 27:2879-2889.

173. Ohad I, Dal Bosco C, Herrmann RG, Meurer J: Photosysytem II proteins PsbL and PsbJ regulate electron flow to the plastoquinone pool. Biochemistry 2004, 43:2297-2308.

174. Yamazaki S, Nomata J, Fujita Y: Differential operation of dual protochlorophyllide reductases for chlorophyll biosynthesis in response to environmental oxygen levels in the cyanobacterium Leptolyngbya boryana. Plant Physiol 2006, 142:911-922.

175. Pruzinská A, Anders I, Aubry S, Schenk N, Tapernoux-Lüthi E, Müller T, Kräutler B, Hörtensteiner S: In vivo participation of red chlorophyll catabolite reductase in chlorophyll breakdown. Plant Cell 2007, 19:369-387.

176. Harada J, Saga Y, Yaeda Y, Oh-Oka H, Tamiaki H: In vitro activity of C-20 methyltransferase, BchU, involved in bacteriochlorophyll $c$ biosynthetic pathway in green sulfur bacteria. FEBS Lett 2005, 579:1983-1987.

177. Long $H$, King PW, Ghirardi ML, Kim K: Hydrogenase/ferredoxin charge-transfer complexes: effect of hydrogenase mutations on the complex association. J Phys Chem A 2009, 113:4060-4067.

178. Spence E, Dunlap WC, Shick JM, Long PF: Redundant pathways of sunscreen biosynthesis in a cyanobacterium. ChemBioChem 2012, 13:531-533.

179. Wegkamp A, van Oorschot W, de Vos WM, Smid EJ: Characterization of the role of para-aminobenzoic acid biosynthesis in folate production by Lactococcus lactis. Appl Environ Microbiol 2007, 73:2673-2681.

180. Sharon I, Tzahor S, Williamson S, Shmoish M, Man-Aharonovich D, Rusch DB, Yooseph S, Zeidner G, Golden SS, Mackey SR, Adir N, Weingart U, Horn D, Venter JC, Mandel-Gutfreund YM, Béjà O: Viral photosynthetic reaction center genes and transcripts in the marine environment. ISME J 2007, 1:492-501.

181. Wang Q, Jantaro S, Lu B, Majeed W, Bailey M, He Q: The high light-inducible polypeptides stabilize trimeric photosystem I complex under high light conditions in Synechocystis PCC 6803. Plant Physiol 2008, 147:1239-1250.

182. Mann NH, Cook A, Millard A, Bailey S, Clokie M: Marine ecosystems: bacterial photosynthesis genes in a virus. Nature 2003, 424:741.

183. Lindell D, Sullivan MB, Johnson ZI, Tolonen AC, Rohwer F, Chisholm SW: Transfer of photosynthesis genes to and from Prochlorococcus viruses. Proc Natl Acad Sci U S A 2004, 101:11013-11018.

184. Mann NH, Clokie MRJ, Millard A, Cook A, Wilson WH, Wheatley PJ, Letarov A, Krisch HM: The genome of S-PM2, a "photosynthetic" T4-type bacteriophage that infects marine Synechococcus strains. J Bacterio/ 2005, 187:3188-3200

185. van Oppen MJH, Leong J-A, Gates RD: Coral-virus interactions: a doubleedged sword? Symbiosis 2009, 47:1-8

186. Nagasaki K, Tomaru Y, Shirai Y, Takano Y, Mizumoto H: Dinoflagellateinfecting viruses. J Mar Biol Assoc UK 2006, 86:469-474.

187. Lohr J, Munn CB, Wilson WH: Characterization of a latent virus-like infection of symbiotic zooxanthellae. Appl Environ Microbiol 2007, 73:2976-2981.
188. Moran NA: Symbiosis as an adaptive process and source of phenotypic complexity. Proc Natl Acad Sci U S A 2007, 104(Suppl 1):8627-8633.

189. Falkowski PG, Dubinsky Z, Muscatine L, Porter JW: Light and the bioenergentics of a symbiotic coral. Bioscience 1984, 34:705-709.

190. Muscatine L, D'Elia CF: The uptake, retention and release of ammonium by reef corals. Limnol Oceanogr 1978, 23:725-734.

191. Rahav O, Dubinsky Z, Achituv Y, Falkowski PG: Ammonium metabolism in the zooxanthellate coral, Stylophora pistillata. Proc R Soc Lond B 1989, 236:325-337.

192. Kawaguti S: Ammonium metabolism of the reef corals. Biol J Okayama Univ 1953, 1:171-176.

193. Kühl M, Cohen Y, Dalsgaard T, Jørgensen BB, Revsbech NP. Microenvironment and photosynthesis of zooxanthellae in scleractinian corals studied with microsensors for O2, $\mathrm{pH}$ and light. Mar Ecol Prog Ser 1995, 117:159-172.

194. Gallon JR: The oxygen sensitivity of nitrogenase: a problem for biochemists and micro-organisms. Trends Biochem Sci 1981, 6:19-23.

195. Webb KL, DuPaul WD, Wiebe W, Sottile W, Johannes RE: Enewetak (Eniwetok) atoll: aspects of the nitrogen cycle on a coral reef. Limnol Oceanogr 1975, 20:198-210.

196. Crossland CJ, Barnes DJ: Dissolved nutrients and organic particulates in water flowing over coral reefs at Lizard Island. Aust J Mar Freshwat Res 1983, 34:835-844.

197. Wilkinson CR, Williams DMB, Sommarco PW, Hogg RW, Trott LA: Rates of nitrogen fixation on coral reefs across the continental shelf of the central Great Barrier Reef. Reef Mar Biol 1984, 80:255-262.

198. Capone DG, Dunham SE, Horrigan SG, Duguay LE: Microbial nitrogen transformations in unconsolidated coral reef sediments. Mar Ecol Prog Ser 1992, 80:75-88.

199. Hewson I, Moisander PH, Morrison AE, Zehr JP: Diazotrophic bacterioplankton in a coral reef lagoon: phylogeny, diel nitrogenase expression and response to phosphate enrichment. ISME J 2007, 1:78-91.

200. Larkum AWD, Kennedy IR, Muller WJ: Nitrogen fixation on a coral reef. Mar Biol 1988, 98:143-155.

201. Shashar $N$, Cohen $Y$, Loya $Y$, Sar N: Nitrogen fixation (acetylene reduction) in stony corals: evidence for coral-bacteria interactions. Mar Ecol Prog Ser 1994, 111:259-264.

202. Lesser MP, Mazel CH, Gorbunov MY, Falkowski PG: Discovery of symbiotic nitrogen-fixing cyanobacteria in corals. Science 2004, 305:997-1000.

203. Mouchka ME, Hewson I, Harvell CD: Coral-associated bacterial assemblages: current knowledge and the potential for climate-driven impacts. Integr Comp Biol 2010, 50:662-674.

204. Olson ND, Ainsworth TD, Gates RD, Takabayashi M: Diazotrophic bacteria associated with Hawaiian Montipora coral: diversity and abundance in correlation with symbiotic dinoflagellates. J Exp Mar Biol Ecol 2009, 371:140-146.

205. Lema KA, Willis BL, Bourne DG: Corals form characteristic associations with symbiotic nitrogen-fixing bacteria. App/ Environ Microbiol 2012, 78:3136-3144.

206. Kellogg CA: Tropical Archaea: diversity associated with the surface microlayer of corals. Mar Ecol Prog Ser 2004, 273:81-88.

207. Wegley L, Edwards R, Rodriguez-Brito B, Liu H, Rohwer F: Metagenomic analysis of the microbial community associated with the coral Porites astreoides. Environ Microbiol 2007, 9:2707-2719.

208. Siboni N, Ben-Dov E, Silvan A, Kushmaro A: Global distribution and diversity of coral-associated Archaea and their possible role in the coral holobiont nitrogen cycle. Environ Microbio/ 2008, 10:2979-2990.

209. Rubio LM, Ludden PW: Biosynthesis of the iron-molybdenum cofactor of nitrogenase. Annu Rev Microbio/ 2008, 62:93-111.

210. Fujita Y, Bauer CE: Reconstitutuion of the light-independent protochlorophyllide reductase from purified Bchl and BchN-BchB subunits. In vitro confirmation of nitrogenase features of a bacteriochlorophyll biosynthesis enzyme. J Biol Chem 2000, 275:23583-23588

211. Sarma R, Barney BM, Hamilton TL, Jones A, Seefeld LC, Peters JW: Crystal structure of the $L$ protein of Rhodobacter sphaeroides light-independent protochlorophyllide reductase with MgADP bound: a homologue of the nitrogenase Fe protein. Biochemistry 2008, 47:13004-13015.

212. Muraki N, Nomata J, Ebata K, Mizoguchi T, Shiba T, Tamiaki H, Kurisu G, Fujita Y: X-ray crystal structure of the light-independent protochlorophyllide reductase. Nature 2010, 465:110-114. 
213. Heyes DJ, Hunter CN: Making light work of enzyme catalysis: protochlorophyllide oxidoreductase. Trends Biochem Sci 2005, 30:642-649.

214. Dean DR, Bolin JT, Zheng L: Nitrogenase metalloclusters: structure, organisation, and synthesis. J Bacteriol 1993, 175:6737-6744.

215. Zheng L, Dean DR: Catalytic formation of a nitrogenase iron-sulfur cluster. J Biol Chem 1994, 269:18723-18726.

216. Shah VK, Stacey G, Brill WJ: Electron transport to nitrogenase. J Biol Chem 1983, 258:12064-12068.

217. Hoover TR, Santero E, Porter S, Kustu S: The integration host factor stimulates interaction of RNA polymerase with FIFA, the transcriptional activator for nitrogen fixation operons. Cell 1990, 63:11-22.

218. Merrick MJ, Edwards RA: Nitrogen control in bacteria. Microbiol Mol Biol Rev 1995, 59:604-622.

219. Kneip C, Lockhart P, Voss C, Maier UG: Nitrogen fixation in eukaryotes - new models for symbiosis. BMC Evol Biol 2007, 7:55.

220. Beman JM, Roberts KJ, Wegley L, Rohwer F, Francis CA: Distribution and diversity of archaeal ammonia momooxygenase genes associated with corals. Appl Environ Microbiol 2007, 73:5642-5647.

221. Campbell WH: Nitrate reductase structure, function and regulation: Bridging the gap between biochemistry and physiology. Annu Rev Plant Physiol Mol Biol 1999, 50:277-303.

222. Einsle O: Structure and function of formate-dependent cytochrome $c$ nitrite reductase, NrfA. Methods Enzymol 2011, 496:399-422.

223. Holden HM, Thoden JB, Raushel FM: Carbamoyl phosphate synthetase: an amazing biochemical odyssey from substrate to product. Cell Mol Life Sci 1999, 56:507-522.

224. Crandall JB, Teece MA: Urea is a dynamic pool of bioavailable nitrogen in coral reefs. Coral Reefs 2012, 31:207-214.

225. Catmull J, Yellowlees D, Miller DJ: NADP+-dependent glutamate dehydrogenase from Acropora formosa: purification and properties. Mar Biol 1987, 95:559-563.

226. Clode PL, Saunders M, Maker G, Ludwig M, Atkins CA: Uric acid deposits in symbiotic marine algae. Plant Cell Environ 2009, 32:170-177.

227. Lancaster JR Jr: Simulation of the diffusion and reaction of endogenously produced nitric oxide. Proc Natl Acad Sci USA 1994, 91:8137-8141.

228. Trapido-Rosenthal H, Zielke S, Owen R, Buxon L, Boeing B, Bhagooli R, Archer J: Increased zooxanthellae nitric oxide synthase activity is associated with coral bleaching. Biol Bull 2005, 208:3-6.

229. Bouchard JN, Yamasaki H: Heat stress stimulates nitric oxide production in Symbiodinium microadriaticum: a possible linkage between nitric oxide and the coral bleaching phenomenon. Plant Cell Physiol 2008, 49:641-652.

230. Perez $S$, Weis V: Nitric oxide and cnidarians bleaching: an eviction notice mediates breakdown of a symbiosis. J Exp Biol 2006, 209:2804-2810.

231. Safavi-Hemami H, Young ND, Doyle J, Llewellyn L, Klueter A: Characterisation of nitric oxide synthase in three cnidarian-dinoflagellate symbioses. Plos One 2010, 5:e10379.

232. Dreyer J, Schleicher M, Tappe A, Schilling K, Kuner T, Kusumawidijaja G, Müller-Esterl W, Oess S, Kuner R: Nitric oxide synthase (NOS)-interacting protein interacts with neuronal NOS and regulates its distribution and activity. J Neurosci 2004, 24:10454-10465.

233. Siebeck O: Photoactivation and depth-dependent UV tolerance in reef coral in the Great Barrier Reef/Australia. Naturwissenschaften 1981, 68:426-428.

234. Reef R, Dunn S, Levy O, Dove S, Shemesh E, Brickner I, Leggat W, HoeghGuldberg O: Photoreactivation is the main repair pathway for UV-induced DNA damage in coral plenulae. J Exp Biol 2009, 212:2760-2766.

235. Anderson S, Zepp R, Machula J, Santavy D, Hansen L, Mueller E: Indicators of UV exposure in corals and their relevance to global climate change and coral bleaching. Hum Ecol Risk Assess 2001, 7:1271-1782.

236. Torregiani JH, Lesser MP: The effects of short-term exposures to ultraviolet radiation in the Hawaiian coral Montipora verrucosa. J Exp Mar Biol Ecol 2007, 340:194-203.

237. Baruch R, Avishai N, Rabinowitz C: UV incites diverse levels of DNA breaks in different cellular compartments of a branching coral species. J Exp Biol 2005, 208:843-848.

238. Hudson CL, Ferrier MD: Assessing ultraviolet radiation-induced DNA damage and repair in field-collected Aiptasia pallida using the comet assay. In Proceedings of the 11th International Coral Reef Symposium. Florida; 2008:7-11.

239. Nesa B, Baird AH, Harii S, Yakovleva I, Hidaka M: Algal symbionts increase DNA damage in coral plenulae exposed to sunlight. Zool Stud 2012, 51:112-117.
240. Vijayavel K, Downs CA, Ostrander GK, Richmond RH: Oxidative DNA damage induced by iron chloride in the larvae of the lace coral Pocillopora damicornis. Comp Biochem Physiol C Toxicol Pharmacol 2012, 155:275-280

241. Lesser MP, Farrell JH: Exposure to solar radiation increases damage to both host tissues and algal symbionts of corals during thermal stress. Coral Reefs 2004, 23:367-377.

242. Polato NR, Vera JC, Baums IB: Gene discovery in the threatened elkhorn coral: 454 sequencing of the Acropora palmata transcriptome. PLoS One 2011, 6:e28634.

243. Lindquist S, Craig EA: The heat-shock proteins. Annu Rev Genet 1988, 22:631-677.

244. Åkerfelt M, Morimoto Rl, Sistonen L: Heat shock factors: Integrators of cell stress, development and lifespan. Nat Rev Mol Cell Biol 2010, 11:545-555.

245. Bosch TCG, Praetzel G: The heat shock response in hydra: immunological relationship of hsp60, the major heat shock protein of Hydra vulgaris, to the ubiquitous hsp70 family. Hydrobiologia 1991, 216-217:513-517

246. Choresh O, Ron E, Loya Y: The 60-kDa heat shock protein (HSP60) of the sea anemone Anemonia virdis; a potential early warning system for environmental change. Mar Biotechnol (NY) 2001, 3:501-508.

247. Bromage E, Carpenter L, Kaattari S, Patterson M: Quantification of coral heat shock proteins from individual coral polyps. Mar Ecol Prog Ser 2009, 376:123-132.

248. Chow AR, Ferrier-Pagès $C$, Khalouei $S$, Reynaud $S$, Brown IR: Increased light intensity induces heat shock protein $\mathrm{Hsp60}$ in coral species. Cell Stress Chaperones 2009, 14:469-476.

249. Venn AA, Quinn J, Jones R, Bodnar A: P-glycoprotein (multi-xenobiotic resistance) and heat shock protein gene expression in the coral Montastraea franksi in response to environmental toxicants. Aquat Toxicol 2009, 93:188-195.

250. Nakamura M, Morita M, Kurihara H, Mitarai S: Expression of $h s p 70$, hsp 90 and $h s f 1$ in the reef coral Acropora digitifera under prospective acidified conditions over the next several decades. Biol Open 2012, 1:75-81.

251. Hayes RL, King CM: Induction of 70-kD heat shock protein in scleractinian corals by elevated temperature: significance for coral bleaching. Mol Mar Biol Biotechnol 1995, 4:36-42.

252. Fang L-S, Huang S-P, Lin K-L: High temperature induces the synthesis of heat-shock proteins and the elevation on intracellular calcium in the coral Acropora grandis. Coral Reefs 1997, 16:127-131.

253. Carpenter LW, Patterson MR, Bromage ES: Water flow influences the spatiotemporal distribution of heat shock protein 70 within colonies of the scleractinian coral Montastrea annularis (Ellis and Solander, 1786) following heat stress: Implications for coral bleaching. J Exp Mar Biol Ecol 2010, 387:52-59.

254. Rosic NN, Pernice M, Dove S, Dunn S, Hoegh-Guldberg O: Gene expression profiles of cytosolic heat shock proteins $\mathrm{Hsp} 70$ and $\mathrm{Hsp} 90$ from symbiotic dinoflagellates in response to thermal stress: possible implications for coral bleaching. Cell Stress Chaperones 2011, 16:69-80.

255. Roth MS, Goericke R, Deheyn DD: Cold induces acute stress but heat is ultimately more deleterious for the reef-building coral Acropora yongei. Sci Rep 2012, 2:240.

256. Aravind L, Anantharaman V, Koonin EV: Monophyly of class I aminoacyl tRNA synthase, USPA, ETFP, photolyase and the PP-ATPase nucleotide-binding domains: Implications for protein evolution in the RNA world. Proteins 2002, 48:1-14.

257. Kültz D: Molecular and evolutionary basis of the cellular stress response. Annu Rev Physiol 2005, 67:225-257.

258. Kvint K, Nachin L, Diez A, Nyström T: The bacterial universal stress protein: functions and regulation. Curr Opin Microbiol 2003, 6:140-145.

259. Kerk D, Bulgrien J, Smith DW, Gribskov M: Arabidopsis proteins containing similarity to the universal stress protein domain of bacteria. Plant Physiol 2003, 131:1209-1219.

260. Forêt $S$, Seneca F, de Jong D, Bieller A, Hemmrich G, Augustin R, Hayward DC, Ball EE, Bosch TC, Agata K, Hassel M, Miller DJ: Phylogenomics reveals an anomalous distribution of USP genes in metazoans. Mol Biol Evol 2011, 28:153-161.

261. DeSalvo MK, Voolstra CR, Sunagawa S, Schwartz JA, Stillman JH, Coffroth MA, Szmant AM, Medina M: Differential gene expression during thermal stress and bleaching in the Caribbean coral Montastraea faveolata. Mol Ecol 2008, 17:3952-3971. 
262. Voolstra CR, Schnetzer J, Penshkin L, Randall CJ, Szmant AM, Medina M: Effects of temperature on gene expression in embryos of the coral Montastraea faveolata. BMC Genomics 2009, 10:627.

263. Sone $H$, Akanuma $H$, Fukuda $T$ : Oxygenomics in environmental stress. Redox Rep 2010, 15:98-114.

264. Pronk TE, Van Someren EP, Stierum RH, Ezendam J, Pennings JLA: Unraveling toxicological mechanisms and predicting toxicity classes with gene dysregulation networks. J App/ Toxicol 2012. doi:10.1002/jat.2800 [Epub ahead of print].

265. Hernández MP, Sullivan WP, Toft DO: The assembly and intermolecular properties of the hsp70-Hop-hsp90 molecular complex. J Biol Chem 2002, 277:38294-38304.

266. Song Y, Masison DC: Independent regulation of Hsp70 and Hsp90 chaperones by Hsp70/Hsp90-organising protein Sti (Hop1). J Biol Chem 2005, 280:34178-34185.

267. Ellis RJ, van der Vies SM: Molecular chaperones. Annu Rev Biochem 1991 60:321-347.

268. Takayama S, Reed JC, Homma S: Heat-shock proteins as regulators of apoptosis. Oncogene 2003, 2003(22):9041-9047.

269. Qiu XB, Shao YM, Miao S, Wang L: The diversity of the DnaJ/Hsp40 family, the crucial partners for Hsp70 chaperones. Cell Mol Life Sci 2006, 63:2560-2570.

270. Ke Q, Costa M: Hypoxia-inducible factor-1 (HIF-1). Mol Pharmacol 2006, 70:1469-1480.

271. Rankin EB, Biju MP, Liu Q, Unger TL, Rha J, Johnson RS, Simon MC, Keith B, Haase VH: Hypoxia-inducible factor-2 (HIF-2) regulates erythropoietin in vivo. J Clin Invest 2007, 117:1068-1077.

272. Levy O, Kaniewska P, Alon S, Eisenberg E, Karako-Lampert S, Bay LK, Reef R, Rodriguez-Lanetty M, Miller DJ, Hoegh-Guldberg O: Complex diel cycles of gene expression in coral-algal symbiosis. Science 2011, 331:175.

273. Glickman MH, Ciechanover A: The ubiquitin-proteasome proteolytic pathway: destruction for the sake of construction. Physiol Rev 2002, 82:373-428.

274. Parsell DA, Lindquist S: The function of heat-shock proteins in stress tolerance: degradation and reactivation of damaged proteins. Annu Rev Genet 1993, 27:437-496.

275. Imai J, Yashiroda H, Maruya M, Yahara I, Tanaka K: Proteasomes and molecular chaperones: cellular machinery responsible for folding and destruction of unfolded proteins. Cell Cycle 2003, 2:585-590.

276. Rosenzweig $\mathrm{R}$, Glickman MH: Forging a proteasome a-ring with dedicated proteasome chaperones. Nat Sruct Mol Biol 2008, 15:218-220.

277. Murata S, Yashiroda H, Tanaka K: Molecular mechanisms of proteasome assembly. Nat Rev Mol Cell Biol 2009, 10:104-115.

278. Bügl H, Fauman EB, Staker BL, Zheng F, Kushner SR, Saper MA, Bardwell JC, Jakob U: RNA methylation under heat shock control. Mol Cell 2000, 6:349-360.

279. Caldas T, Binet E, Bouloc P, Costa A, Desgres J, Richarme G: The FtsJ/RrmJ heat shock protein of Escherichia coli is a $23 \mathrm{~S}$ ribosomal RNA methyl transferase. J Biol Chem 2000, 275:16414-16419.

280. Shimuta T, Nakano K, Yamaguchi Y, Ozaki S, Fujimitsu K, Matsunaga C, Noguchi K, Emoto A, Katayama T: Novel heat shock protein HspQ stimulates degradation of mutant DnaA protein in Escherichia coli. Genes Cells 2004, 9:1151-1166.

281. Kitagawa M, Wada C, Yoshioka S, Yura T: Expression of ClpB, an analog of the ATP-dependent protease regulatory subunit in Escherichia coli, is controlled by a heat shock sigma factor (sigma 32). J Bacteriol 1991, 173:4247-4253.

282. Squires C, Squires CL: The Clp proteins: proteolysis or molecular chaperones? J Bacteriol 1992, 174:1081-1085.

283. Lupas AN, Koretke KK: Bioinformatic anatysis of ClpS, a protein module involved in prokaryotic and eukaryotic protein degradation. J Struct Biol 2003, 141:77-83.

284. Maillard RA, Chistol G, Sen M, Righini M, Tan J, Kaiser CM, Hodges C, Martin A, Bustamante C: ClpX(P) generates mechanical force to unfold and translocate its protein substrates. Cell 2011, 145:459-469.

285. Horwitz J: Alpha-crystallin can function as a molecular chaperone. Proc Natl Acad Sci U S A 1992, 89:10449-10453.

286. Rao PV, Horwitz J, Zigler JS: a-Crystallin, a molecular chaperone, forms a stable complex with carbonic anhydrase upon heat denaturation. Biochem Biophys Res Commun 1993, 190:786-793.

287. Carricart-Ganivet JP, Cabanillas-Terán N, Cruz-Ortega I, Blanchon P: Sensitivity of calcification to thermal stress varies among genera of massive reef-building corals. PLoS One 2012, 7:e32859.
288. Taylor JL, Wieczorek A, Keyser AR, Grover A, Flinkstrom R, Karls RK, Bielefeldt-Ohmann H, Dobos KM, Izzo AA: HspX-mediated protection against tuberculosis depends on the chaperoning of a mycobacterial molecule. Immunol Cell Biol 2012, 90:945-954.

289. Santo Ede O, Alves N Jr, Dias GM, Mazotto AM, Vermelho A, Vora GJ, Wilson B, Beltran VH, Bourne DG, Le Roux F, Thompson FL: Genomic and proteomic analysis of the pathogen Vibrio coralliilyticus reveals a diverse virulence repertoire. ISME J 2011, 5:1471-1483.

290. Kimes NE, Grim CJ, Johnson WR, Hasan NA, Tall BD, Kothary MH, Kiss H, Munk AC, Tapia R, Green L, Detter C, Bruce DC, Brettin TS, Colwell RR, Morris $P J:$ Temperature regulation of virulence factors in the pathogen Vibrio coralliilyticus. ISME J 2012, 6:835-846.

291. Zhang T, Kraus WL: SIRT1-dependent regulation of chromatin and transcription: linking $\mathrm{NAD}^{+}$metabolism and signaling to the control of cellular functions. Biochim Biophys Acta 1804, 2010:1666-1675.

292. Liu TF, Yoza BK, El Gazzar M, Vachharajani VT, McCall CE: NAD+-dependent SIRT1 deacetylase participates in epigenetic reprogramming during endotoxin tolerance. J Biol Chem 2011, 286:9856-9864.

293. Katada S, Imhof A, Sassone-Corsi P: Connecting threads: epigenetics and metabolism. Cell 2012, 148:24-28.

294. Lim JH, Lee YM, Chun YS, Chen J, Kim JE, Park JW: Sirtuin 1 modulates cellular responses to hypoxia by deacetylating hypoxia-inducible Factor 1a. Mol Cell 2010, 38:864-878.

295. Lee JH, Song MY, Song EK, Kim EK, Moon WS, Han MK, Park JW, Kwon KB, Park BH: Overexpression of SIRT1 protects pancreatic beta-cells against cytokine toxicity by suppressing the nuclear factor-kappaB signalling pathway. Diabetes 2009, 58:344-351.

296. Wang F, Nguyen M, Qin FX, Tong Q: SIRT2 deacetylates FOXO3a in response to oxidative stress and caloric restriction. Aging Cell 2007, 6:505-514.

297. Shi T, Wang F, Stieren E, Tong Q: SIRT3, a mitochondrial sirtuin deacetylase, regulates mitochondrial function and thermogenesis in brown adipocytes. J Biol Chem 2005, 280:13560-13567.

298. Nasrin N, Wu X, Fortier E, Feng Y, Baré OC, Chen S, Ren X, Wu Z, Streeper RS, Bordone L: SIRT4 regulates fatty acid oxidation and mitochondrial gene expression in liver and muscle cells. J Biol Chem 2010, 285:31995-32002.

299. Du J, Zhou Y, Su X, Yu JJ, Khan S, Jiang H, Kim J, Woo J, Kim JH, Choi BH, He B, Chen W, Zhang S, Cerione RA, Auwerx J, Hao Q, Lin H: Sirt5 is a NAD-dependent protein lysine demalonylase and desuccinylase. Science 2011, 334:806-809.

300. Mostoslavsky R, Chua KF, Lombard DB, Pang WW, Fisher MR, Gellon L, Liu P, Mostoslavsky G, Franco S, Murphy MM, Mills KD, Patel P, Hsu JT, Hong AL, Ford E, Cheng HL, Kennedy C, Nunez N, Bronson R, Frendewey D, Auerbach W, Valenzuela D, Karow M, Hottiger MO, Hursting S, Barrett JC, Guarente L, Mulligan R, Demple B, Yancopoulos GD, et al: Genomic instability and aging-like phenotype in the absence of mammalian SIRT6. Cell 2006, 124:315-329.

301. Luo J, Nikolaev AY, Imai S, Chen D, Su F, Shiloh A, Guarente L, Gu W: Negative control of p53 by Sir2alpha promotes cell survival under stress. Cell 2001, 107:137-148

302. Vakhrusheva O, Smolka C, Gajawada P, Kostin S, Boettger T, Kubin T, Braun T, Bober E: Sirt7 increases stress resistance of cardiomyocytes and prevent apoptosis and inflammatory cardiomyopathy in mice. Circ Res 2008, 102:703-710.

303. O'Halloran TV, Culotta VC: Metallochaperones, an intracellular shuttle service for metal ions. J Biol Chem 2000, 275:25057-25060.

304. Lin SJ, Culotta VC: The ATX1 gene of Saccharomyces cerevisiae encodes a small metal homeostasis factor that protects cells against reactive oxygen toxicity. Proc Natl Acad Sci U S A 1995, 92:3784-3788.

305. Culotta VC, Klomp LWJ, Strain J, Casareno RLB, Krems B, Gitlin JD: The copper chaperone for superoxide dismutase. J Biol Chem 1997, 272:23469-23472

306. Wong PC, Waggoner D, Subramaniam JR, Tessarollo L, Bartnikas TB, Culotta VC, Price DL, Rothstein J, Gitlin JD: Copper chaperone for superoxide dismutase is essential to activate mammalian $\mathrm{Cu} / \mathrm{Zn}$ superoxide dismutase. Proc Natl Acad Sci USA 2000, 97:2886-2891.

307. Shick JM, Lesser MP, Dunlap WC, Stochaj WR, Chalker BE, Wu Won J: Depth-dependent responses to solar ultraviolet radiation and oxidative stress in the zooxanthellate coral Acropora microphthalma. Mar Biol 1995, 122:41-51.

308. Lesser MP, Stochaj WR, Tapley DW, Shick JM: Bleaching in coral-reef anthozoans: effects of irradiance, ultraviolet radiation, and temperature on the activities of protective enzymes against active oxygen. Coral Reefs 1990, 8:225-232 
309. Crowe JH, Carpenter JF, Crowe LM: The role of vitrification in anhydrobiosis. Annu Rev Physiol 1998, 60:73-103.

310. Sola-Penna M, Meyer-Fernandes JR: Stabilization against thermal inactivation promoted by sugars on enzyme structure and function: Why is trehalose more effective than other sugars? Arch Biochem Biophys 1998, 360:10-14.

311. Veron JEN: Corals of Australia and the Indo-Pacific. Singapore: University of Hawaii Press; 1993:644.

312. Shick JM: Solar UV and oxidative stress in algal-animal symbioses. In Frontiers of Photobiology. Edited by Shima A, Ichihashi M, Fujiwara Y, Takebe H. Amsterdam: Elsevier Science Publishers; 1993:561-564.

313. Turrens JF: Mitochondrial formation of reactive oxygen species. J Physiol 2003, 552:335-344

314. Lesser MP: Oxidative stress causes coral bleaching during exposure to elevated temperatures. Coral Reefs 1997, 16:187-192.

315. Brown BE, Downs CA, Dunn RP, Gibb SW: Exploring the basis of thermotolerance in the reef coral Goniastrea aspera. Mar Ecol Prog Ser 2002, 242:119-120.

316. Halliwell B: Reactive species and antioxidants. Redox biology is a fundamental theme of aerobic life. Plant Physiol 2006, 141:312-322.

317. Liñán-Cabello MA, Lesser MP, Flores-Ramírez LA, Zenteno-Savín T, Reyes-Bonilla $\mathrm{H}$ : Oxidative stress in coral-photobiont communities. In Oxidative Stress in Aquatic Ecosystems. Edited by Abele D, Vázquez-Medina JP, Zenteno-Savín T. Chichester: Wiley-Blackwell; 2011:127-138.

318. Jönsson TJ, Lowther WT: The peroxiredoxin repair proteins. Subcell Biochem 2007, 44:115-141.

319. Cheng Z, Zhang J, Ballou DP, Williams CH Jr: Reactivity of thioredoxin as a protein thiol-disulfide oxidoreductase. Chem Rev 2011, 111:5768-5783.

320. Arnér ESJ, Holmgren A: Physiological function of thioredoxin and thioredoxin reductase. Eur J Biochem 2000, 267:6102-6109.

321. Ainsworth TD, Hoegh-Guldberg O: Cellular processes of bleaching in the Mediterranean coral Oculina patagonica. Coral Reefs 2008, 27:593-597.

322. Kenkel CD, Aglyamova G, Alamaru A, Bhagooli R, Capper R, Cunning R, De Villers A, Haslun JA, Hédouin L, Keshavmurthy S, Kuehl KA, Mahmoud H, McGinty ES, Montoya-Maya PH, Palmer CV, Pantile R, Sánchez JA, Schils T, Silverstein RN, Squiers LB, Tang PC, Goulet TL, Matz MV: Development of gene expression markers of acute heat-light stress in reef-building corals of the genus Porites. PLoS One 2011, 6:e26914

323. Wang C, Youle RJ: The role of mitochondria in apoptosis. Annu Rev Genet 2009, 43:95-118.

324. Richier S, Sabourault C, Courtiade J, Zucchini N, Allemand Furla P: Oxidative stress and apoptotic events during thermal stress in the sea anemone, Anemone viridis. FEBS J 2006, 273:4186-4198.

325. Lasi M, Pauly B, Schmidt N, Cikala M, Stiening B, Käsbauer T, Zenner G, Popp T Wagner A, Knapp RT, Huber AH, Grunert M, Söding J, David CN, Böttger A: The molecular cell death machinery in the simple cnidarian Hydra includes an expanded caspase family and pro- and anti-apoptotic $\mathrm{Bcl}-2$ proteins. Cell Res 2010, 20:812-825.

326. Baker AC, Starger CJ, McClanahan TR, Glynn PW: Coral reefs: corals' adaptive response to climate change. Nature 2004, 430:741.

327. McClanahan T: The relationship between bleaching and mortality of common corals. Mar Biol 2004, 144:1239-1245.

328. Ainsworth TD, Wasmund K, Ukani L, Seneca F, Yellowlees D, Miller D, Leggat W: Defining the tipping point. A complex cellular life/death balance in corals in response to stress. Sci Rep 2011, 1:160.

329. Tchernov D, Kvitt H, Haramaty L, Bibby TS, Gorbunov MY, Rosenfeld H, Falkowski PG: Apoptosis and the selective survival of host animals following thermal bleaching in zooxanthellate corals. Proc Natl Acad Sci U S A 2011, 108:9905-9909.

330. Pernice M, Dunn SR, Miard T, Dufour S, Dove S, Hoegh-Guldberg O: Regulation of apoptotic mediators reveals dynamic responses to thermal stress in the reef building coral Acropora millepora. PLoS One 2011, 6:e16095.

331. David CN, Schmidt N, Schade M, Pauly B, Alexabdrova O, Böttger A: Hydra and the evolution of apoptosis. Integr Comp Biol 2005, 45:631-638.

332. Zmasek CM, Zhang Q, Ye Y, Godzik A: Surprising complexity of the ancestral apoptosis network. Genome Biol 2007, 8:R226.

333. Siepp S, Wittig K, Stiening B, Böttger A, Leitz T: Morphogenesis in Hydractina echinata (Cnidaria) is capase-dependent. Int J Dev Biol 2006, 50:63-70.

334. Dunn SR, Weis VM: Apoptosis as a post-phagocytic mechanism in a coraldinoflagellate mutualism. Environ Microbiol 2009, 11:268-276.
335. Adrian C, Brumatti G, Martin SJ: Apoptosomes: protease activation platforms to die for. Trends Biochem Sci 2006, 31:243-247.

336. Yang $X$, Khosravi-Far R, Chang HY, Baltimore D: Daxx, a novel Fas-binding protein that activates JNK and apoptosis. Cell 1997, 89:1067-1076.

337. Cohen $O$, Inbal B, Kissil JL, Raveh T, Berissi H, Spivak-Kroizaman T, Feinstein E, Kimchi A: DAP-kinase participates in TNF-a- and Fas-induced apoptosis and its function requires the death domain. J Cell Biol 1999, 146:141-148.

338. Lee JH, Rho SB, Chun T: Programmed cell death 6 (PDCD6) protein interacts with death associated protein kinase 1 (DAPk1): additive effect on apoptosis via caspase-3 dependent pathway. Biotechnol Lett 2005, 27:1011-1015

339. Kaiser WJ, Upton JW, Long AB, Livingstone-Rosanoff D, Daley-Bauer LP, Hakem R, Caspary T, Mocarski ES: RIP3 mediates the embryonic lethality of caspase-8 deficient mice. Nature 2011, 471:368-372.

340. Krumschnabel G, Sohm B, Bock F, Manzl C, Villunger A: The enigma of caspase-2: the layman's view. Cell Death Differ 2009, 16:195-207.

341. Khorchid A, Ikura M: How calpain is activated by calcium. Nat Struct Biol 2002, 9:239-341.

342. Janssens S, Timel A, Lippens T, Tschopp J: PIDD mediates NF-kB activation response to DNA damage. Cell 2005, 123:1079-1092.

343. Wolenski FS, Garbati MR, Lubinski TL, Traylor-Knowles N, Dresselhaus E, Stefanik DJ, Goucher H, Finnerty JR, Gilmore TD: Characterisation of the core elements of the NF-KB signalling pathway of the sea anemone Nematostella vectensis. Mol Cell Biol 2011, 31:1076-1087.

344. Dunn SR, Phillips WS, Spatafora JW, Green DR, Wies VM: Highly conserved caspase and $\mathrm{BCl}-2$ homologues from the sea anemone Aptaisia pallida: lower metazoans for the study of apoptosis evolution. J Mol Evol 2006, 63:95-107.

345. Knowlton N, Rohwer F: Multispecies microbial mutualisms on coral reefs: the host as a habitat. Am Nat 2003, 162(Suppl):S51-S62

346. Littman RA, Willis BL, Pfeffer C, Bourne DG: Diversities of coral-associated bacteria differ with location, but not species, for three acroporid corals on the Great Barrier Reef. FEMS Microbiol Ecol 2009, 68:152-153.

347. Rohwer F, Seguritan V, Azam F, Knowlton N: Diversity and distribution of coral-associated bacteria. Mar Ecol Prog Ser 2002, 243:1-10.

348. Sogin ML, Morrison HG, Huber JA, Mark Welch D, Huse SM, Neal PR, Arrieta $J M$, Herndl GJ: Microbial diversity in the deep sea and the unexplored "rare biosphere". Proc Natl Acad Sci U S A 2006, 103:12115-12120.

349. Sunagawa S, DeSalvo MK, Voolstra CR, Reyes-Bermudez A, Medina M: Identification and gene expression analysis of a taxonomically restricted cysteine-rich protein family in reef-building corals. PLoS One 2009, 4:e4865.

350. Rosenberg E, Koren O, Reshef L, Efrony R, Zilber-Rosenberg I: The role of microorganisms in coral health, disease, and evolution. Nat Rev Microbiol 2007, 5:355-362

351. Wild C, Huettel M, Klueter A, Kremb SG, Rasheed MY, Jørgensen BB: Coral mucus formation functions as an energy carrier and particle trap in the reef ecosystem. Nature 2004, 428:66-70.

352. Garren M, Azam F: Corals shed bacteria as a potential mechanism of resilience to organic matter enrichment. ISME J 2012, 6:1159-1165.

353. Bourne DG, Garren M, Work TM, Rosenberg E, Smith GW, Harvell CD: Microbial disease and the coral holobiont. Trends Microbiol 2009, 17:554-562.

354. Reshef L, Koren O, Loya Y, Zilber-Rosenberg I, Rosenberg E: The coral probiotic hypothesis. Environ Microbiol 2006, 8:2068-2073.

355. Vollmer SV, Kline DI: Natural disease resistance in threatened staghorn corals. PLoS One 2008, 3:e3718.

356. Carroll SP, Hendry AP, Reznick DN, Fox CW: Evolution on ecological time scales. Funct Ecol 2007, 21:387-393.

357. van Oppen MJH, Souter P, Howells EJ, Heyward A, Berkelmans R: Novel genetic diversity through somatic mutations: fuel for adaptation of reef corals? Diversity 2011, 3:405-423.

358. Mydlarz LD, McGinty ES, Hravell CD: What are the physiological and immunological responses of coral to climate warming and disease? J Exp Biol 2010, 213:934-945.

359. Hamada M, Shoguchi E, Shinzato C, Kawashima T, Miller DJ, Satoh N: The complex NOD-like receptor repertoire of the coral Acropora digitifera includes novel domain combinations. Mol Biol Evol 2013, 30:167-176

360. Boyes DC, Nam J, Dangl JL: The Arabidopsis thaliana RPM1 disease resistance gene product is a peripheral plasma membrane protein that 
is degraded coincident with the hypersensitive response. Proc Natl Acad Sci U S A 1998, 95:15849-15854.

361. Axtell MJ, Stakawicz BJ: Initiation of RPS2-specified disease resistance in Arabidopsis is coupled to the AvrRpt2-directed elimination of RIN4. Cell 2003, 112:369-377

362. Edreva A: Pathogenesis-related proteins: research progress in the last 15 years. Gen Appl Plant Physiol 2005, 31:105-124.

363. Palmer CV, Mydlarz LD, Willis BL: Evidence of an inflammatory-like response in non-normally pigmented tissues of two scleractinian corals. Proc Biol Sci 2008, 275:2687-2693.

364. Mydlarz LD, Couch CS, Weil E, Smith G, Harvell CD: Immune defenses of healthy, bleached and diseased Montastraea faveolata during a natural bleaching event. Dis Aquat Organ 2009, 87:67-78.

365. Palmer C, Bythwll JC, Willis BL: A comparative study of phenoloxidase activity in diseased and bleached colonies of the coral Acropora millepora. Dev Comp Immunol 2011, 35:1098-1101.

366. Cascales E, Christie PJ: The versatile bacterial type IV secretion systems. Nat Rev Microbiol 2003, 1:137-149.

367. Juhas M, Crook DW, Hood DW: Type IV secretion systems: tools of bacterial gene transfer and virulence. Cell Microbiol 2008, 10:2377-2386.

368. Technau U, Rudd S, Maxwell P, Gordon PM, Saina M, Grasso LC, Hayward DC, Sensen CW, Saint R, Holstein TW, Ball EE, Miller DJ: Maintenance of ancestral complexity and non-metazoan genes in two basal cnidarians. Trends Genet 2005, 21:633-639.

369. Khalturin K, Hemmeich G, Fraune S, Augustin R, Bosch TC: More than just orphans: are taxonomically-restricted genes important in evolution? Trends Genet 2009, 25:404-413.

370. Forêt S, Knack B, Houliston E, Momose T, Manuel M, Quéinnec E, Hayward DC, Ball EE, Miller DJ: New tricks with old genes: the genetic basis of novel cnidarian traits. Trends Genet 2010, 26:154-158.

371. Bergh O, Børsheim KY, Bratbak G, Heldal M: High abundance of viruses found in aquatic environments. Nature 1989, 340:467-468.

372. Børsheim KY, Bratbak G, Heldal M: Enumeration and biomass estimation of planktonic bacteria and viruses by transmission electron microscopy. Appl Environ Microbiol 1990, 56:352-356.

373. Breitbart M, Salamon P, Andressen B, Mahaffy JM, Segall AM, Mead D, Azam $F$, Rohwer F: Genomic analysis of uncultured marine viral communities. Proc Natl Acad Sci U S A 2002, 99:14250-14255.

374. Williamson SJ, Rusch DB, Yooseph S, Halpern AL, Heidelberg KB, Glass Jl, Andrews-Pfannkoch C, Fadrosh D, Miller CS, Sutton G, Frazier M, Venter JC: The Sorcerer II Global Ocean Sampling Expedition: metagenomic characterization of viruses within aquatic microbial samples. PLoS One 2008, 3:e1456.

375. Bouvy M, Combe M, Bettarel Y, Dupuy C, Rochelle-Newall E, Charpy L: Uncoupled viral and bacterial distributions in coral reef waters of the Tuamotu Archipelago (French Polynesia). Mar Pollut Bull 2012, 65:506-515.

376. Dinsdale EA, Pantos O, Smriga S, Edwards RA, Angly F, Wegley L, Hatay M, Hall D, Brown E, Haynes M, Krause L, Sala E, Sandin SA, Thurber RV, Willis BL, Azam F, Knowlton N, Rohwer F: Microbial ecology of four coral atolls in the Northern Line Islands. PLoS One 2008, 3:e1584.

377. Seymour JR, Patten N, Bourne DG, Mitchell JG: Spatial dynamic of virus-like particles and heterotrophic bacteria within a shallow coral reef system. Mar Ecol Prog Ser 2005, 288:1-8.

378. Patten NL, Seymour JR, Mitchell JG: Flow cytometric analysis of virus-like particles and heterotrophic bacteria within coral-associated reef water. J Mar Biol Assoc UK 2006, 86:563-566.

379. Leruste A, Bouvier T, Bettarel Y: Enumerating viruses in coral mucus. Appl Environ Microbiol 2012, 78:6377-6379.

380. Davy JE, Patten NL: Morphological diversity of virus-like particles within the surface microlayer of scleractinian corals. Aquat Microb Ecol 2007, 47:37-44.

381. Patten NL, Harrison PL, Mitchell JG: Prevalence of virus-like particles within the staghorn scleractinian coral (Acropora muricata) from the Great Barrier Reef. Coral Reefs 2008, 27:569-580.

382. Wilson WH, Francis I, Ryan K, Davy SK: Temperature induction of viruses in symbiotic dinoflagellates. Aquat Microb Ecol 2001, 25:99-102.

383. Wilson WH, Dale AL, Davy JE, Davy SK: An enemy within? Observation of virus-like particles in reef corals. Coral Reefs 2005, 24:145-148.

384. Davy SK, Burchett SG, Dale AL, Davies P, Davy JE, Muncke C, HoeghGuldberg O, Wilson WH: Viruses: agents of coral disease? Dis Aquat Org 2006, 69:101-110
385. Correa AM, Welsh RM, Vega Thurber RL: Unique nucleocytoplasmic dsDNA and +ssRNA viruses are associated with dinoflagellate endosymbionts of corals. ISME J 2013, 7:13-27.

386. Marhaver KL, Edwards RA, Rohwer F: Viral communities associated with health and bleached corals. Environ Microbiol 2008, 10:2277-2286.

387. Vega Thurber RL, Barott KL, Hall D, Liu H, Rodriguez-Mueller B, Desnues C, Edwards RA, Haynes M, Angly FE, Wegley L, Rohwer FL: Metagenomic analysis indicates that stressors induce production of herpes-like viruses in the coral Porites compressa. Proc Natl Acad Sci U S A 2008, 105:18413-18418.

388. Bettarel Y, Thuy NT, Huy TQ, Hoang PK, Bouvier T: Observation of virus-like particles in thin sections of the bleaching scleractinian coral Acropora cytherea. J Mar Biol Assoc UK 2013, 93:909-912.

389. Wilson WH: Coral viruses. In Studies in Viral Ecology, Volume 2. Animal host systems. Edited by Hurst CJ. Hoboken: Wiley; 2011:143-152.

390. Kuznetsov SG, Bosch TC: Self/nonself recognition in Cnidaria: contact of allergenic tissue does not result in elimination of nonself cells in Hydra vulgaris. Zool (Jena) 2003, 106:109-116.

391. Rinkevich B: Allorecognition and xenorecognition in reef corals: a decade of interactions. Hydrobiologia 2004, 530-531:443-450.

392. Rinkevich B: Neglected biological features in cnidarians self-nonself recognition. Adv Exp Med Biol 2012, 738:46-59.

393. Bosch TCG: The path less explored: innate immune reactions in cnidarians. In Nucleic Acids and Molecular Biology. Volume 21. Innate Immunity of Plants, Animals and Humans. Edited by Heine H. Heidelberg: Springer-Verlag Berlin; 2008:27-42

394. Wood-Charlson EM, Hollingsworth LL, Krupp DA, Weis VM: Lectin/glycan interactions play a role in recognition in a coral/dinoflagellate symbiosis. Cell Microbiol 2006, 8:1985-1993.

395. Hemmrich G, Miller DJ, Bosch TCG: The evolution of immunity: a low-life perspective. Trends Immunol 2007, 28:449-454.

396. Miller DJ, Hemmrich G, Ball EE, Hayward DC, Khalturin K, Funayama N, Agata K, Bosch TC: The innate immune repertoire in Cnidaria - ancestral complexity and stochastic gene loss. Genome Biol 2007, 8:R59.

397. Kvennefors ECE, Leggat W, Hoegh-Guldberg O, Degnan BM, Barnes AC: An ancient and variable mannose-binding lectin from the coral Acropora millepora binds both pathogens and symbionts. Dev Comp Immunol 2008, 32:1582-1592

398. Bosch TCG, Augustin R, Anton-Erxleben F, Fraune S, Hemmrich G, Zill H, Rosenstiel P, Jacobs G, Schreiber S, Leippe M, Stanisak M, Grötzinger J, Jung S, Podschun R, Bartels J, Harder J, Schröder JM: Uncovering the evolutionary history of innate immunity: the simple metazoan Hydra uses epithelial cells for host defence. Dev Comp Immunol 2009, 33:559-569.

399. Dunn SR: Immunorecognition and immunoreceptors in the Cnidaria. Invertebrate Surviv J 2009, 6:7-14.

400. Rosenstiel P, Philipp EER, Schreiber S, Bosch TCG: Evolution and function of innate immune receptors - insights from marine invertebrates. J Innate Immun 2009, 1:291-300.

401. Augustin R, Bosch TCG: Cnidarian immunity: a tale of two barriers. Adv Exp Med Biol 2010, 708:1-16.

402. Mydlarz $L D$, Jones $L E$, Harvell CD: Innate immunity, environmental drivers, and disease ecology of marine and freshwater invertebrates. Annu Rev Ecol Evol Syst 2006, 37:251-288.

403. Widner WR, Wickner RB: Evidence that the SKI antiviral system of Saccharomyces cerevisiae acts by blocking expression of viral mRNA. Mol Cell Biol 1993, 13:4331-4341.

404. Brown JT, Bai X, Johnson AW: The yeast antiviral proteins Ski2p, Ski3p and Ski8p exist as a complex in vivo. RNA 2000, 6:449-457.

405. Houseley J, Tollervey D: The many pathways of RNA degradation. Cell 2009, 136:763-776.

406. Arraiano CM, Matos RG, Barbas A: RNase II: The finer details of the modus operandi of a molecular killer. RNA Biol 2010, 7:276-281.

407. Samuel CE: Antiviral actions of interferon. Interferon-regulated proteins and their surprisingly selective antiviral activities. Virology 1991, 183:1-11.

408. Haller O, Kochs G, Webe F: The interferon response circuit: Induction and suppression by pathogenic viruses. Virology 2006, 344:119-130.

409. Fensterl V, Sen GC: Interferons and viral infections. Biofactors 2009, 35:14-20.

410. Taniguchi T, Ogasawara K, Takaoka A, Tanaka N: IRF family of transcription factors as regulators of host defense. Annu Rev Immunol 2001, 19:623-655. 
411. Dornan D, Eckert M, Wallace M, Shimizu H, Ramsay E, Hupp TR, Ball KL: Interferon regulatory factor 1 binding to p300 stimulates DNA-dependent acetylation of p53. Mol Cell Biol 2004, 24:10083-10098.

412. Salkowski CA, Barber SA, Detore GR, Vogel SN: Differential dysregulation of nitric oxide production in macrophages with targeted disruptions in INF regulatory factor-1 and -2 genes. J Immunol 1996, 156:3107-3110.

413. Weisz A, Marx P, Sharf R, Appella E, Driggers PH, Ozato K, Levi BZ: Human interferon consensus sequence binding protein is a negative regulator of enhancer elements common to interferon-inducible genes. J Biol Chem 1992, 267:25589-25596.

414. Espert L, Degols G, Gongora C, Blondel D, Williams BR, Silverman RH, Mechti N: ISG20, a new interferon-induced RNase specific for single-stranded RNA, defines an alternative antiviral pathway against RNA genomic viruses. J Biol Chem 2003, 278:16151-16158.

415. Taylor GA, Jeffers M, Largaespada DA, Jenkins NA, Copeland NG, Vande Woude GF: Identification of a novel GTPase, the inducibly expressed GTPase, that accumulates in response to interferon $ү$. J Biol Chem 1996, 271:20399-20405.

416. Haller $O$, Staeheli $P$, Kochs $G$ : Interferon-induced $M x$ proteins in antiviral host defense. Biochimie 2007, 89:812-818.

417. Pichlmair A, Schulz O, Tan CP, Rehwinkel J, Kato H, Takeuchi O, Akira S, Way M, Schiavo G, Reise Sousa C: Activation of MDA5 requires higher-order RNA structures generated during virus infection. J Virol 2009, 83:10761-10769.

418. Deblandre GA, Marinx OP, Evans SS, Majjaj S, Leo O, Caput D, Huez GA, Wathelet MG: Expression cloning of an interferon-inducible $17-\mathrm{kDa}$ membrane protein implicated in the control of cell growth. $J$ Biol Chem 1995, 270:23860-23866.

419. Farrar MA, Schreiber RD: The molecular cell biology of interferon-gamma and its receptor. Annu Rev Immunol 1993, 11:571-611.

420. Blasius AL, Giurisato E, Cella M, Schreiber RD, Shaw AS, Colonna M: Bone marrow stromal cell antigen is a specific marker of type 1 IFN-producing cells in the native mouse, but a promiscuous cell surface antigen following IFN stimulation. J Immuno/ 2006, 177:3260-3265.

421. Seth RB, Sun L, Ea CK, Chen ZJ: Indentification and characterization of MAVS, a mitochondrial antiviral signaling protein that activates NF-kappaB and IRF3. Cell 2005, 122:669-682.

422. Crook NE, Clem RJ, Miller LK: An apoptosis-inhibiting baculovirus gene with a zinc finger-like motif. J Virol 1993, 67:2168-2174.

423. Yang YL, Li XM: The IAP family: endogenous caspase inhibitors with multiple biological activities. Cell Res 2000, 10:169-177.

424. Spear PG, Longnecker R: Herpesvirus entry: an update. J Virol 2003, 77:10179-10185.

425. Nemerow GR, Wolfert R, McNaughton ME, Cooper NR: Identification and characterization of the Epstein-Barr virus receptor on human $B$ lymphocytes and its relationship to the C3d component receptor (CR2). J Virol 1985, 55:347-351.

426. Wu FK, Garcia JA, Harrich D, Gaynor RB: Purification of the human immunodeficiency virus type 1 enhancer and TAR binding proteins EBP-1 and UBP-1. EMBO J 1988, 7:2117-2130.

427. Lu Y, Qian XY, Krug RM: The influenza virus NS1 protein: a novel inhibitor of pre-mRNA splicing. Genes Dev 1994, 8:1817-1828.

428. Hale BG, Randall RE, Ortín J, Jackson D: The multifunctional NS1 protein of influenza A viruses. J Gen Virol 2008, 89:2359-2376.

429. Freundlich M, Ramani N, Mathew E, Sirko A, Tsui P: The role of intergration host factor in gene expression in Escherichia coli. Mol Microbiol 1992, 6:2557-2563.

430. Tailor CS, Willett BJ, Kabat D: A putative cell surface receptor for anemia-inducing feline leukemia virus subgroup $C$ is a member of a transporter superfamily. J Virol 1999, 73:6500-6505.

431. Bard-Chapeau EA, Jeyakani J, Kok CH, Muller J, Chua BQ, Gunaratne J, Batagov A, Jenjaroenpun P, Kuznetsov VA, Wei CL, D'Andrea RJ, Bourque G, Jenkins NA, Copeland NG: Ecotopic viral integration site 1 (EVI1) regulates multiple cellular processes important for cancer and is a synergistic partner for FOS protein in invasive tumors. Proc Natl Acad SCi US A 2012, 109:2168-2173.

432. Turk T, Kem WR: The phylum Cnidaria and investigations of its toxins and venoms until 1990. Toxicon 2009, 54:1031-1037.

433. Anderluh G, Macek P: Cytolytic peptide and protein toxins from sea anemones (Anthozoa: Actiniaria). Toxicon 2002, 40:111-124.

434. Frazão B, Vasconcelos V, Antunes A: Sea anemone (Cnidaria, Anthozoa, Actiniaria) toxins: an overview. Mar Drugs 2012, 10:1812-1851.
435. Weston AJ, Chung R, Dunlap WC, Morandini AC, Marques AC, Mourada-Silva AM, Ward M, Padilla G, da Silva LF, Andreakis N, Long PF: Proteomic characterisation of toxins isolated from nematocysts of the South Atlantic jellyfish Olindias sambaquiensis. Toxicon 2013. doi:10.1016/j. toxicon.2013.05.002

436. Brinkman DL, Burnell JN: Biochemical and molecular characterisation of cubozoan protein toxins. Toxicon 2009, 54:1162-1173.

437. Brinkman DL, Aziz A, Loukas A, Potriquet J, Seymour J, Mulvenna J: Venom proteome of the Box Jellyfish Chironex fleckeri. PLoS One 2012, 7:e47866.

438. Leppla SH: Anthrax toxin edema factor: a bacterial adenylate cyclase that increases cyclic AMP concentrations of eukaryotic cells. Proc Natl Acad Sci USA 1982, 79:3162-3166.

439. Tang WJ, Guo Q: The andenylyl cyclase activity of anthrax edema factor. Mol Aspt Med 2009, 30:423-430

440. Van Delden C, Iglewski BH: Cell-to-cell signaling and Pseudomonas aeruginosa infections. Emerg Infect Dis 1998, 4:551-560.

441. Yates SP, Merrill AR: Elucidation of eukaryotic elongation factor-2 contact sites within the catalytic domain of Pseudomonas aeruginosa exotoxin A. Biochem J 2004, 379:563-572.

442. Hinnebusch BJ, Rudolph AE, Cherepanov P, Dixon JE, Schwan TG, Forsberg A: Role of Yersinia murine toxin in survival of Yersinia pestis in the midgut of the flea vector. Science 2002, 296:733-735.

443. Fagerlund A, Lindbäck T, Storset AK, Granum PE, Hardy SP: Bacillus cereus Nhe is a pore-forming toxin with structural and functional properties similar to the ClyA (HlyE, SheA) family of haemolysins, able to induce osmotic lysis in epithelia. Microbiology 2008, 154:693-704.

444. Marrack P, Kappler J: The staphylococcal enterotoxins and their relatives. Science 1990, 248:705-711.

445. Voth DE, Ballard JD: Clostridium difficile toxins: mechanism of action and role in disease. Clin Microbiol Rev 2005, 18:247-263.

446. Di Pierro M, Lu R, Uzzau S, Wang W, Margaretten K, Pazzani C, Maimone F, Fasano A: Zonula occludens toxin structure-function analysis. Identification of the fragment biologically active on tight junctions and of the zonulin receptor binding domain. J Biol Chem 2001, 276:19160-19165.

447. Blaser MJ: Helicobacter pylori and the parthenogenesis of gastroduodenal inflammation. J Infect Dis 1990, 161:626-633.

448. Miller VL, Taylor RK, Mekalanos JJ: Cholera toxin transcriptional activator toxR is a transmembrane DNA binding protein. Cell 1987, 48:271-279.

449. Childers BM, Klose KE: Regulation of virulence in Vibrio cholerae: the ToxR regulon. Future Microbiol 2007, 2:335-344.

450. Lanka $E$, Wilkins BM: DNA processing reactions in bacterial conjugation. Annu Rev Biochem 1995, 64:141-169.

451. Iredell JR, Manning PA: The toxin-coregulated pilus of Vibrio cholerae 01: a model for type A pilus biogenesis? Trends Microbiol 1994, 2:187-192.

452. Kim Y, Wang X, Zhang X-S, Grigoriu S, Page R, Peti W, Wood TK: Escherichia coli toxin/antitoxin pair MqsR/MqsA regulate toxin CspD. Environ Microbiol 2010, 12:1105-1121.

453. Yamanaka K, Zheng W, Crooke E, Wang YH, Inouye M: CspD, a novel DNA replication inhibitor induced during stationary phase in Escherichia coli. Mol Microbiol 2001, 39:1572-1584.

454. French CT, Panina EM, Yeh SH, Griffith N, Arambula DG, Miller JF: The Bordella type III secretion system effector BteA contains a conserved $\mathrm{N}$-terminal motif that guides bacterial virulence factors to lipid rafts. Cell Microbiol 2009, 11:1735-1749.

455. Espinosa A, Alfano JR: Disabling surveillance: bacterial type III secretion effectors that suppress innate immunity. Cell Microbiol 2004, 6:1027-1040.

456. Deane JE, Roversi P, Cordes FS, Johnson S, Kenjale R, Daniell S, Booy F, Picking WD, Picking WL, Blocker AJ, Lea SM: Molecular model of a type III secretion system needle: implication for host cell sensing. Proc Natl Acad Sci U S A 2006, 103:12529-12533.

457. Jungo F, Bougueleret $L$, Xenarios I, Poux S: The UniProtKB/Swiss-Prot Tox-Prot program: a central hub of integrated venom protein data. Toxicon 2012, 60:551-557.

458. Chang D, Duda TF Jr: Extensive and continuous duplication facilitates rapid evolution and diversification of gene family. Mol Biol Evol 2012, 29:2019-2029.

459. Wong ESW, Belov K: Venom evolution through gene duplication. Gene 2012, 496:1-7

460. Moura-da-Silva AM, Furlan MS, Caporrino MC, Grego KF, Portes-Junior JA, Clissa PB, Valente RH, Magalhães GS: Diversity of metalloproteinases in 
Bothrops neuwiedi snake venom transcripts: evidences for recombination between different classes of SVMPs. BMC Genet 2011, 12:94.

461. Dutertre S, Jin AH, Kaas Q, Jones A, Alewood PF, Lewis RJ: Deep venomics reveals the mechanism for expanded peptide diversity in cone snail venom. Mol Cell Proteomics 2012, 12:312-329.

462. Starcevic A, Long PF: Diversification of animal venom peptides - were jellyfish amongst the first combinatorial chemists? ChemBiochem 2013. doi:10.1002/cbic.201300305.

463. Fabricius KE: Factors determining the resistance of coral reefs to eutrophication: A review and conceptual model. In Coral Reefs: An Ecosystem in Transition. Edited by Dubinsky Z, Stambler N. Heidelberg: Springer Dordrecht; 2011:493-508.

464. Ferrier-Pagès C, Gatusso J-P, Dallot S, Jaubert J: Effect of nutrient enrichment on growth and photosynthesis of the zooxanthellate coral Stylophora pistillata. Coral Reefs 2000, 19:103-113.

465. Kramarsky-Winter E, Downs CA, Downs A, Loya Y: Cellular responses in the coral Stylophora pistillata exposed to eutrophication from fish mariculture. Evol Ecol Res 2009, 11:1-21.

466. Goldstone JV, Hamdoun A, Cole BJ, Howard-Ashby M, Nebert DW, Scally M, Dean M, Epel D, Hahn ME, Stegeman JJ: The chemical defensome: environmental sensing and response genes in the Strongylocentrotus purpuratus genome. Dev Biol 2006, 300:366-384.

467. Sarasquete C, Segner H: Cytochrome P4501A (CYP1A) in teleostean fishes. A review of immunohistochemical studies. Sci Total Environ 2000, 247:313-332.

468. Gassman NJ, Kennedy CJ: Cytochrome P-450 content and xenobiotic metabolizing enzyme activity in the scleractinian coral, Favia fragum (Esper). Bull Mar Sci 1992, 50:320-330.

469. Garcia E, Ramos R, Bastidas C: Presence of cytochrome P450 in the Carribean corals Siderastrea sidereal and Montastaea faveolata. Cinc Mar 2005, 31:23-30.

470. Ramos R, Garcia E: Induction of mixed-function oxygenase system and antioxidant enzymes in the coral Montastraea faveolata exposure to benzo(a)pyrene. Comp Biochem Physiol C. Toxicol Pharmacol 2007, 144:348-355.

471. Rougée L, Downs CA, Richmond RH, Ostrander GK: Alteration of normal cellular profiles in the scleractinian coral (Pocillopora damicornis) following laboratory exposure to fuel oil. Environ Toxicol Chem 2006, 25:3181-3187

472. Goldstone JV: Environmental sensing and response genes in Cnidaria: the chemical defensome in the sea anemone Nematostella vectensis. Cell Biol Toxicol 2008, 24:483-502.

473. Khanal SK, Xie B, Tompson ML, Sung S, Ong SK, Van Leeuwent J: Fate, transport and biodegradation of natural estrogens in the environmental and engineering systems. Environ Sci Technol 2006, 40:6537-6546.

474. Snyder SA, Westerhoff P, Yoon Y, Sedlak DL: Pharmaceuticals, personal care products, and endocrine disruptors in water: Implications for the water industry. Environ Eng Sci 2003, 20:449-469.

475. Goksøyr A: Endocrine disruptors in the marine environment: mechanisms of toxicity and their influence on reproductive processes in fish. J Toxicol Environ Health A 2006, 69:175-184.

476. Tarrant AM, Atkinson MJ, Atkinson S: Effects of steroidal estrogens on coral growth and reproduction. Mar Ecol Prog Ser 2004, 269:121-129.

477. Hayes JD, Flanagan JU, Jowsey IR: Glutathione transferases. Annu Rev Pharmacol Toxicol 2005, 45:51-88.

478. Karntanut W, Pascoe D: The toxicity of copper, cadmium and zinc to four different Hydra (Cndaria: Hydrozoan). Chemosphere 2002, 47:1059-1064

479. Gadelha JR, Ferreira VAM, Abreu SN, Soars AMVM, Morgado FMR: Experimental mercury bioaccumulation trends in sea anemone Actinia equina exposed to chlor-alkali industry effluent contaminated water. In Interdisciplinary Studies on Environmental Chemistry - Biological Responses to Chemical Contaminants: From Molecular to Community Level. Volume 3. Edited by Hamamura N, Suzuki S, Mendo S, Barroso CM, Iwata H, Tanabe S. Tokyo: Terrapub; 2010:149-157.

480. Alutoin S, Boberg J, Nyström M, Tedengren M: Effects of the multiple stressors copper and reduced salinity on the metabolism of the hermatypic coral Porites lutea. Mar Environ Res 2001, 52:289-299.

481. Nyström M, Nordemar I, Tedengren M: Simultaneous and sequential stress from increased temperature and copper on the metabolism of the hermatypic coral Porites cylindrica. Mar Biol 2001, 138:1225-1231.

482. Mitchelmore CL, Verde EA, Weis VM: Uptake and partitioning of copper and cadmium in the coral Pocillopora damicornis. Aquat Toxicol 2007, 85:48-56.
483. Bielmyer GK, Grosell M, Bhagooli R, Baker AC, Langdon C, Gillette P, Capo TR: Differential effects of copper on three species of scleractinian corals and their algal symbionts (Symbiodinium spp.). Aquat Toxicol 2010, 97:125-133.

484. Bastidas C, Garcia EM: Sublethal effects of mercury and its distribution in the coral Porites astreoides. Mar Ecol Prog Ser 2004, 267:133-143.

485. Farina O, Ramos R, Bastidas C, García E: Biochemical response of cnidarian larvae to mercury and benzo(a)pyrene exposure. Bull Environ Contam Toxicol 2008, 81:553-557.

486. Negri AP, Howard AJ: Inhibition of coral fertilisation and larval metamorphosis by tributyltin and copper. Mar Environ Res 2001, 51:17-27.

487. Reichelt-Brushett AJ, Harrison PL: The effect of selected trace metals on the fertilzation success of several scleractinian coral species. Coral Reefs 2005, 24:524-534.

488. Victor S, Richmond RH: Effect of copper on fertilisation success in the reef coral Acropora surculosa. Mar Pollut Bull 2005, 50:1448-1451.

489. Jones RJ: Zooxanthellae loss as a bioassay for assessing stress in corals. Mar Ecol Prog Ser 1997, 149:163-171.

490. Reitzel AM, Sullivan JC, Traylor-Knowles N, Finnerty JR: Genomic survey of candidate stress-response genes in the estuarine anemone Nematostella vectensis. Biol Bull 2008, 214:233-254.

491. Steele RA, Opella SJ: Structures of the reduced and mercury-bound forms of MerP, the periplasmic protein from the bacterial mercury detoxification system. Biochemistry 1997, 36:688-6895.

492. Mukhopadhyay R, Rosen BP: Arsenate reductases in prokaryotes and eukaryotes. Environ Health Perspect 2002, 110:745-748.

493. Hyakawa T, Kobayashi Y, Cui X, Hirano S: A new metabolic pathway of arsenite:arsenic-glutathione complexes are substrates for human arsenic methyltransferase Cyt19. Arch Toxicol 2005, 79:183-191.

494. Price RE, London J, Wallaschläger D, Ruiz-Chancho MJ, Pichler T: Enhanced bioaccumulation and biotransformation of As in coral reef organisms surrounding a marine shallow-water hydrothermal vent system. Chem Geol 2012. doi:10.1016/j.chemgeo.2012.02.023.

495. David CP: Heavy metal concentrations in growth bands of corals: a record of mine tailings input through time (Marinduque Island, Philippines). Mar Poll Bull 2003, 46:187-196.

496. Pessi G, Hass D: Transcriptional control of the hydrogen cyanide biosynthetic genes $h c n A B C$ by the anaerobic regulator ANR and the quorum-sensing regulators LasR and RhIR in Pseudomonas aeruginosa. J Baceriol 2000, 182:6940-6949.

497. Bird A: Perceptions of epigenetics. Nature 2007, 447:396-398

498. Jablonka E, Lamb MJ: Epigenetic inheritance in evolution. J Evol Biol 1998 11:159-183.

499. Mirouze M, Paszkowski J: Epigenetic contribution to stress adaptation in plants. Curr Opin Plant Biol 2011, 14:267-274.

500. Strahl BD, Allis CD: The language of covalent histone modifications. Nature 2000, 403:41-45.

501. Wang M, Mok MW, Harper H, Lee WH, Min J, Knapp S, Oppermann U, Marsden B, Schapira M: Structural genomics of histone tail recognition. Bioinformatics 2010, 26:2629-2630.

502. Goll MG, Bestor TH: Eukaryotic cytosine methyltransferases. Annu Rev Biochem 2005, 74:481-514.

503. Slokin RK, Martienssen R: Transposable elements and the epigenetic regulation of the genome. Nat Rev Genet 2007, 8:272-285.

504. Wolffe AP, Jones PL, Wade PA: DNA demethylation. Proc Natl Acad Sci U S A 1999, 96:5894-5896.

505. Ooi SK, Bestor TH: The colorful history of active DNA demethylation. Cell 2008, 133:1145-1148.

506. Zhu JK: Active DNA demethylation mediated by DNA glycosylases. Annu Rev Genet 2009, 43:143-166.

507. Branco MR, Ficz G, Reik W: Uncovering the role of 5hydroxymethylcytosine in the epigenome. Nat Rev Genet 2011, 13:7-13.

508. Guo JU, Su Y, Zhong C, Ming GL, Song H: Hydroxylation of 5-methylcytosine by TET1 promotes active DNA demethylation in the adult brain. Cell 2011, 145:423-434.

509. Roberts SB, Gavery MR: Is there a relationship between DNA methylation and phenotypic plasticity in invertebrates? Front Physiol 2012, 2:116.

510. Brown BE, Cossins AR: The potential for temperature acclimatisation of reef corals in the face of climate change. In Coral Reefs: And Ecosystem in Transition. Edited by Dubinski Z, Stambler N. Berlin: Springer-Verlag; 2011:421-433. 
511. Gilbert SF, McDonald E, Boyle N, Buttino N, Gyi L, Mai M, Prakash N, Robinson J: Symbiosis as a source of selectable epigenetic variation: taking the heat for the big guy. Phil Trans R Soc B 2010, 365:671-678

512. Sievers F, Wilm A, Dineen DG, Gibson TJ, Karplus K, Li W, Lopez R, McWilliam H, Remmert M, Söding J, Thompson JD, Higgins DG: Fast, scalable generation of high-quality protein multiple sequence alignments using Clustal Omega. Mol Syst Biol 2011, 7:539.

513. Apache-Tomcat. [http://tomcat.apache.org/download-70.cgi]

doi:10.1186/1471-2164-14-509

Cite this article as: Dunlap et al: KEGG orthology-based annotation of the predicted proteome of Acropora digitifera: ZoophyteBase - an open access and searchable database of a coral genome. BMC Genomics 2013 14:509.

\section{Submit your next manuscript to BioMed Central and take full advantage of:}

- Convenient online submission

- Thorough peer review

- No space constraints or color figure charges

- Immediate publication on acceptance

- Inclusion in PubMed, CAS, Scopus and Google Scholar

- Research which is freely available for redistribution 